\title{
DEPENDENT THEORIES AND THE GENERIC PAIR CONJECTURE
}

\author{
SAHARON SHELAH
}

\begin{abstract}
We try to understand complete types over a somewhat saturated model of a complete first order theory which is dependent (previously called NIP), by "decomposition theorems for such types". Our thesis is that the picture of dependent theory is the combination of the one for stable theories and the one for the theory of dense linear order or trees (and first we should try to understand the quite saturated case). As a measure of our progress, we give several applications considering some test questions; in particular we try to prove the generic pair conjecture and do it for measurable cardinals.
\end{abstract}

Date: November 15, 2013.

2010 Mathematics Subject Classification. Primary 03C45; Secondary: 03C55.

Key words and phrases. model theory, first order theories, classification theory, dependent theories, the generic pair conjecture.

The author would like to thank the Israel Science Foundation for partial support of this research (Grant No. 242/03). I would like to thank Alice Leonhardt for the beautiful typing. First version as F756 was 2006/Feb/14; and $900-2007 / J a n / 10$. Publication 900. 


\section{Annotated Content}

$\S 0 \quad$ Introduction, pg. 3

$\S 1 \quad$ Non-splitting construction, pg. 10

[For $\kappa$-saturated $M$ and $N$ such that $M \prec N$ we try to analyze $N$ over $M$ by finding $M_{1}, N_{1}$ such that $M \prec M_{1} \prec N_{1}, M \prec N \prec N_{1}$ and both $M_{1} / M, N_{1} / N$ are understood but in opposite ways. The first similar in some sense to the stable situation, the second to the situation for order.]

$\S 2$ The type decomposition theorem, pg. 19

[For $\kappa$-saturated $M \prec \mathfrak{C}$ and $\bar{d} \in \mathfrak{C}$ of length $<\theta^{+}$we try to analyze the type $\operatorname{tp}(\bar{d}, M)$ in two steps - pseudo stable and tree-like one. This is the main aim of the section (and a major part of the paper). It is done by looking at $K_{\ell}$ and $\operatorname{mxK}_{\lambda, \kappa, \theta}^{\ell}$. A consequence which fulfilled to some extent the aim is the Type Decomposition Theorem (2.4). As a second consequence we give a characterization of " $M$ is exactly $\kappa$-saturated, $\kappa>\operatorname{cf}(\kappa)>$ $|T|$ ", see 2.2. In fact, we deal a little with singular exact saturation per se. "Unfortunately" there are independent (complete first order theories) $T$ which has no model with singular exact saturation, see 2.23. But the existence of an indiscernible set for dependent $T$ suffice (see 2.26 under instances of $\mathrm{GCH}$ ) and has a neat characterization. Also, if $p$ is a complete 1-type over a model $M$ of $T$ which is quite saturated then $p$ has a spectrum in a suitable sense, see 2.31.]

$\S 3 \quad$ Existence of strict decomposition, pg. 37

[E.g. here complete types over a saturated model $M$ of cardinality $\kappa$, a measurable cardinal, is analyzed. What we get is a better decomposition theorem (the strict one).]

$\S 4$ Consequences of strict decomposition, pg. 44

[We start by sufficient conditions for a sequence being indiscernible. For a measurable cardinal $\kappa(>|T|)$ we confirm the structure half of the generic pair conjecture. Toward this, if we have the consequences of $\S 3$ we can analyze generic pairs of models of $T$ in $\kappa$. In a slightly simplified formulation this means: if $2^{\kappa}=\kappa^{+},\left(\kappa=\kappa^{<\kappa}>|T|\right), M_{\alpha}$, a model of $T$ of cardinality $\kappa$ for $\alpha<\kappa^{+}$is $\prec$-increasing continuous, $M=\cup\left\{M_{\alpha}: \alpha<\kappa^{+}\right\}$is $\kappa^{+}$saturated, then for a club $E$ of $\kappa^{+}$for all $\alpha<\beta$ belonging to $\{\delta \in E: \delta$ has cofinality $\kappa\}$ the pair $\left(M_{\beta}, M_{\alpha}\right)$ has the same isomorphism type. In fact for $\kappa_{1}<\kappa_{2}$ we get $\mathbb{L}_{\infty, \kappa_{1}}\left(\tau_{T}\right)$-equivalence, so we have a derived first order theory. For the proof we show that an increasing (short) sequence of so called strict $(\kappa, \theta)$-decompositions has a limit.] 


\section{$\S 0$. INTRODUCTION}

We first give a page of introductory remarks for non-logicians to clarify notions and to motivate working on dependent theories. The classical center of model theory is investigating elementary classes, i.e. we fix a vocabulary $\tau$ (i.e. a set of predicates and function symbols), for a $\tau$-structure $M$ let $\operatorname{Th}(M)$ be the set of first order sentences which $M$ satisfies, a complete first order theory $T$ is $\operatorname{Th}(M)$ for some $\tau$-model $M$. We fix $T$ and $\tau=\tau_{T}$ and investigate $\tau$-models of $T$, i.e. $\tau$-structures $M$ such that $T=\operatorname{Th}(M)$; about other contexts, see e.g. Sh:E53.

Let $M, N$ denote such structures and they are called models (of $T$ ). Let $\bar{a}, \bar{b}, \bar{c}, \bar{d}$ denote sequences of elements of such models and $\varphi(\bar{x})$ or $\varphi(\bar{x}, \bar{y})$ denote members of $\mathbb{L}(\tau)$, i.e. the set of first order formulas in this vocabulary but we allow $\bar{x}$ to be infinite though the formula is finite so only finitely many $x_{\ell}, y_{j}$ are relevant.

Let $M \models \varphi[\bar{a}]$ mean that the model $M$ satisfies the formula $\varphi(\bar{x})$ under the substitution $\bar{x} \mapsto \bar{a}$ (so $\bar{a}, \bar{x}$ have the same length).

The right notion of sub-models is $\prec$, being elementary submodel where $M \prec N$ iff $M \subseteq N$ and for every $\varphi(\bar{x}) \in \mathbb{L}(\tau)$ and $\bar{a} \in{ }^{\ell g(\bar{x})} M$ we have $M \models \varphi[\bar{a}]$ iff $N \models \varphi[\bar{a}]$.

Recall that an ordinal is the isomorphism type of a well ordering (which is a linear order for which every non-empty set has a first member). But we identify an ordinal with the set of smaller ordinals. Also a cardinal is an ordinal $\lambda$ with no smaller ordinal of the same power. Here saying " $x$ is a cardinal" means " $x$ is an infinite cardinal" if not said otherwise. Let $\aleph_{\alpha}$ be the $\alpha$-th infinite cardinal and the cardinality $|\mathscr{U}|$ of a set $\mathscr{U}$ is the minimal ordinal of the same power.

Let the successor $\lambda^{+}$of a cardinal $\lambda$ be $\aleph_{\alpha+1}$ when $\lambda=\aleph_{\alpha}$.

We say $E$ is a closed subset of the limit cardinal $\gamma$ when $E \subseteq \gamma$ and $\delta<\gamma \wedge \delta=$ $\sup (\delta \cap E) \Rightarrow \delta \in E$ and $E$ is called unbounded when $(\forall \alpha<\gamma)(\exists \beta)(\alpha \leq \beta \in E)$, " $E$ is a club of $\gamma$ " is the shorthand for " $E$ is a closed unbounded subset of $\gamma$ ".

For an ordinal $\alpha$ let $\operatorname{cf}(\alpha)=\min \{|C|: C$ an unbounded subset of $\alpha\}=$ $\min \{\operatorname{otp}(C): C$ a closed unbounded subset of $\alpha\}$; we say $\alpha$ is regular if $\alpha=\operatorname{cf}(\alpha)$ is infinite (hence is a cardinal), now recall (see e.g. Jec03) that if $\alpha$ is a limit ordinal (e.g. a cardinal) then $\operatorname{cf}(\alpha)$ is regular, and every cardinal of the form $\lambda^{+}$is regular. When $\operatorname{cf}(\delta)>\aleph_{0}$ we say " $S \subseteq \delta$ is stationary" when $S \cap E \neq \emptyset$ for every club $E$ of $\delta$.

A central notion is type; for $A \subseteq M$ and $\bar{a}$ a sequence from $M$ let $\operatorname{tp}(\bar{a}, A, M)$ be the set $\{\varphi(\bar{x}, \bar{b}): \bar{\varphi}(\bar{x}, \bar{y}) \in \mathbb{L}(\tau), \bar{b}$ a sequence from $A$ and $M \models \varphi[\bar{a}, \bar{b}]\}$. We may write $a$ instead of $\langle a\rangle$.

Let

$$
\begin{aligned}
& \mathbf{S}^{\alpha}(A, M)=\{\operatorname{tp}(\bar{a}, A, N): \quad \text { for some } N, \bar{a} \text { we have } \\
& M \prec N, \bar{a} \text { a sequence of length } \alpha \text { from } N \text { \} } \\
& \mathbf{S}^{\alpha}(M)=\mathbf{S}^{\alpha}(M, M) .
\end{aligned}
$$

By this we can define another central notion. $M$ is $\kappa$-saturated $\underline{\text { iff for every } A \subseteq}$ $M,|A|<\kappa$ and $p \in \mathbf{S}^{1}(A, M)$ some $a \in M$ realizes $p$ in $M$ which means $p=$ $\operatorname{tp}(a, A, M)$. We say the model $M$ is saturated when it is $\kappa$-saturated and of cardinality $\kappa$. Let $\operatorname{EC}_{\lambda}(T)$ be the class of models of $T$ of cardinality $\lambda$. 
It is classically known that for $\lambda \geq|T|$, (assuming $2^{\lambda}=\lambda^{+}$, mostly done here for transparency) there is a saturated member of $\mathrm{EC}_{\lambda^{+}}(T)$, it is unique up to isomorphism, and the union of an $\prec$-increasing chain of saturated members of $\mathrm{EC}_{\lambda^{+}}(T)$ of length $\lambda^{+}$is a saturated member of $\mathrm{EC}_{\lambda^{+}}(T)$. On the background so far, see e.g. Chang-Keisler [CK73.

A major theme of the author's work is trying to find natural dividing lines (i.e. properties) in the family of first order complete $T$, a criterion for natural is having both "inside definition" by formulas and "outside definition" by properties of the class of its models. That is, such a property is interesting as a dividing line when we have consequences for those with the property and for those without it; see e.g. Sh:E53, $\S(1 \mathrm{~A})]$.

A major such dividing line is " $T$ is stable" which holds iff $(*)_{T}^{1}$ iff $(*)_{T}^{2}$ where

$(*)_{T}^{1}$ for some $\varphi(\bar{x}, \bar{y}) \in \mathbb{L}\left(\tau_{T}\right)$, model $M$ of $T$ and $\bar{a}_{n} \in{ }^{(\ell g(\bar{x}))} M, \bar{b}_{n} \in{ }^{(\ell g(\bar{y}))} M$ for $n<\omega$ we have $n<m \Leftrightarrow M \models \varphi\left[\bar{a}_{n}, \bar{b}_{m}\right]$

$(*)_{T}^{2}$ for every $\lambda \geq|T|$ and limit ordinal $\delta \leq \lambda$ of cofinality $>|T|$, the union of any $\prec$-increasing chain of length $\delta$ of saturated models of $T$ of cardinality $\lambda$ is saturated.

Another major dividing line is " $T$ is superstable" which holds iff

$(*)_{T}^{3}$ like $(*)_{T}^{2}$ allowing any limit ordinal $\delta$.

On this and the relevant history, see e.g. [Sh:c].

The property we deal with here is " $T$ is dependent", also called " $T$ is NIP", where its negation, " $T$ is independent" or " $T$ has the independence property" means

$(*)_{T}^{4}$ there are $\varphi(\bar{x}, \bar{y}) \in \mathbb{L}\left(\tau_{T}\right)$, a model $M$ of $T$ and $\bar{a}_{u} \in{ }^{(\ell g(\bar{x}))} M, \bar{b}_{n} \in{ }^{(\ell g(\bar{y}))} M$ for $u \subseteq \omega, n<\omega$ such that $n \in u \Leftrightarrow M \models \varphi\left[\bar{a}_{u}, \bar{b}_{n}\right]$.

What is the motivation to investigate this dividing line? First, it has a nice, simple definition, parallel to the one for stable theories. Second, it is a much wider class than that of the stable theories; also, extremely important for many, whereas infinite fields with stable first order complete theory are hard to come by (algebraically closed and separably closed are the only known ones), there are many important fields with dependent first order complete theory (the $p$-adics and many of the power series fields). Third, there are some results on it indicating it is not unreasonable to hope there is a rich theory on it to be discovered.

On background on dependent theories, see [Sh:715], Sh:783.

Let $T$ be a fixed first order complete theory. For transparency, till 0.1 , we assume G.C.H., i.e. $2^{\kappa}=\kappa^{+}$for every infinite cardinal $\kappa$ and consider only $\lambda$ regular $>|T|$. Let $\bar{M}=\left\langle M_{\alpha}: \alpha<\lambda^{+}\right\rangle$be an $\prec$-increasing continuous sequence of models of $T$ of cardinality $\lambda$ with $M$ being saturated where $M:=\cup\left\{M_{\alpha}: \alpha<\lambda^{+}\right\}$. Now $M$ is unique (up to isomorphism, for each $\lambda$ ) and though for a given $M, \bar{M}$ is not unique, for any two such sequences $\bar{M}^{\prime}, \bar{M}^{\prime \prime}$ there is a closed unbounded subset $E$ of $\lambda^{+}$ and isomorphism $f$ from $M^{\prime}=\cup\left\{M_{\alpha}^{\prime}: \alpha<\lambda^{+}\right\}$onto $M^{\prime \prime}=\cup\left\{M_{\alpha}^{\prime \prime}: \alpha<\lambda\right\}$ such that $f$ maps $M_{\delta}^{\prime}$ onto $M_{\delta}^{\prime \prime}$ for every $\delta \in E$. 
So it is natural to ask $(\lambda>|T|$ regular and $E$ varies on closed unbounded subsets of $\lambda^{+}$)

$\odot_{1}$ what 11 is $\mathbf{n}_{\lambda}(T):=\operatorname{Min}_{E}\left|\left\{M_{\delta} / \cong: \delta \in E\right\}\right| ?$ where $M_{\delta} / \cong$ is the isomorphism type of $M_{\delta}$. When is $\mathbf{n}_{\lambda}(T)$ equal to one?

Now (see [Sh:868]):

$\odot_{2} \mathbf{n}_{\lambda}(T)=1$ iff $T$ is superstable

$\odot_{3}$ for countable $T, \mathbf{n}_{\lambda}(T)=2 \underline{\text { iff }} T$ is strictly stable (i.e. $T$ is stable, not superstable)

$\odot_{4}$ given an ordinal $\gamma$, for $\lambda$ large enough $\mathbf{n}_{\lambda}(T)=|\gamma+1|$ if $T$ is stable and $\kappa(T)=\aleph_{\gamma}$ (recalling that for a stable $T, \kappa(T)$ is cardinal $\leq|T|^{+}$, so for countable $T$ it is $\aleph_{0}$ or $\aleph_{1}$ )

$\odot_{5}$ if $T$ is unstable, $\lambda=\aleph_{\gamma}$ then $\mathbf{n}_{\lambda}(T) \geq|\gamma+1|$.

[Why? Because for some closed unbounded subset $E$ of $\lambda^{+}$, if $\delta \in E$ then $M_{\delta}$ is $\operatorname{cf}(\delta)$-saturated but not $(\operatorname{cf}(\delta))^{+}$-saturated hence $\left[\delta_{1}, \delta_{2} \in E \wedge \operatorname{cf}\left(\delta_{1}\right) \neq \operatorname{cf}\left(\delta_{2}\right) \Rightarrow\right.$ $M_{\delta_{1}} ¥ M_{\delta_{2}}$.]

Hence it is natural to replace $\mathbf{n}_{\lambda}(T)$ by:

$\odot_{6}$ let $\mathbf{n}_{\lambda, \kappa}(T)=\operatorname{Min}_{E} \mid\left\{M_{\delta} / \cong: \delta \in E\right.$ and $\left.\operatorname{cf}(\delta)=\kappa\right\} \mid$ when $\lambda>\kappa=\operatorname{cf}(\kappa)$ (as above $E$ varies on the clubs of $\lambda^{+}$).

Below we use $\mathbf{n}_{\lambda, \kappa}(T)$ only when $\lambda=\operatorname{cf}(\lambda)>|T|+\kappa \wedge \kappa=\operatorname{cf}(\kappa)$ and remember that for simplicity we are assuming G.C.H.

Now (see Sh:868]):

$\odot_{7}$ if $T$ is stable then $\mathbf{n}_{\lambda, \kappa}(T)=1$.

It is natural to ask whether this characterizes stable theories. The answer is no, in fact, by an example everyone knows (by [Sh:877, §1]):

$\odot_{8} \mathbf{n}_{\lambda, \kappa}(T)=1$ for $T=\operatorname{Th}(\mathbb{Q},<)$, the theory of dense linear orders with neither first nor last element, so $\lambda=\lambda^{<\lambda}>\kappa=\operatorname{cf}(\kappa)$.

During the proof we analyze $p \in \mathbf{S}\left(M_{\alpha}\right), M_{\alpha}$ saturated, of course, only when $p \neq$ $\operatorname{tp}\left(a, M_{\alpha}, M_{\alpha}\right)$ for $a \in M_{\alpha}$. So $M_{\alpha}$ is a linear order and $p$ induces a cut $\left(C_{p}^{-}, C_{p}^{+}\right)$ of $M_{\alpha}$, i.e. $C_{p}^{-}=\left\{a \in M_{\alpha}:(a<x) \in p\right\}$ is an initial segment of $M_{\alpha}$ and its compliment, $\left\{a \in M_{\alpha}:(a<x) \notin p\right\}$ is an end segment. This gives a pair of cofinalities, $\left(\mu_{p}^{-}, \mu_{p}^{+}\right), \mu_{p}^{-}$the cofinality of the linear order $C_{p}^{-}$and $\mu_{p}^{+}$the cofinality of the inverse of $C_{p}^{+}$.

Now

$(*)_{8.1}$ if $\mu_{p}:=\min \left\{\mu_{p}^{-}, \mu_{p}^{+}\right\}<\lambda$, then the type is determined by any subset of $M_{\alpha}$ of cardinality $\mu_{p}$ such that:

- the set is unbounded in $C_{p}^{-}$if $\mu_{p}=\mu_{p}^{-}$and

\footnotetext{
${ }^{1}$ We can present the problem differently, about the existence of (variations of) $(\lambda, \kappa)$-limit models (so $2^{\lambda}=\lambda^{+}$is no longer necessary, by forcing this is equivalent). Also, instead of the function $\mathbf{n}$ getting the value $\lambda^{+}$we can consider saying for some club no two relevant cases are isomorphic. This does not make a real difference but we find the present choice has more transparent presentation.
} 
- the set is unbounded from below in $C_{p}^{+}$if $\mu_{p}=\mu_{p}^{+}$.

$(*)_{8.2}$ if $\mu_{p}=\lambda$, and we expand $M_{\alpha}$ by the (unary) relation $C_{p}^{-}$, we still get a saturated model.

Next considering $\odot_{7}+\odot_{8}$ you may think that for every $T$ we get $\mathbf{n}_{\lambda, \kappa}(T)=1$, but (Sh:877, 2.3(2)] implies directly that):

$\odot_{9} \mathbf{n}_{\lambda, \kappa}(T)=\lambda^{+}$if $T$ is Peano arithmetic

moreover, this holds for quite many theories $T$ (by [Sh:877, $\S 2]$ ):

$\odot_{10} \mathbf{n}_{\lambda, \kappa}(T)=\lambda^{+}$if $T$ has the strong independence property (see Sh:72, i.e. for some first order formula $\varphi(x, y),\langle\varphi(M, a): a \in M\rangle$ is an independent sequence of subsets of $M$, see Definition (0.6).

For me this rings a bell and strengthens a suspicion - maybe the dividing line is $T$ independent/ $T$ dependent, indeed (by Sh:877, §2]):

$\odot_{11} \mathbf{n}_{\lambda, \kappa}(T)=\lambda^{+}$if $T$ is independent, $\lambda$ a successor cardinal.

We try here to address the complement, the structure side. This calls for analyzing appropriate $\prec$-increasing continuous sequence $\bar{M}=\left\langle M_{i}: i \leq \kappa\right\rangle$ of models of $T$ of cardinality $\lambda$. Clearly in the relevant cases they "increase fast enough" and $M_{i}$ is saturated for $i$ non-limit. Now among such sequences, is it not reasonable to first deal with the case of length 2 ?

This leads to the generic pair conjecture which says that for $\lambda=\lambda^{<\lambda}>|T|$, we have $T$ is independent iff $\mathbf{n}_{\lambda, 2}(T)=\lambda^{+}$where:

$\odot_{12} \mathbf{n}_{\lambda, 2}^{*}(T):=\operatorname{Min}_{E} \mid\left\{\left(M_{\beta}, M_{\alpha}\right) / \cong: \alpha<\beta\right.$ belongs to $E$ and $\operatorname{cf}(\alpha)=\lambda=$ $\operatorname{cf}(\beta)\} \mid$.

Note that in defining $\mathbf{n}_{\lambda, \kappa}(T), \kappa \in \operatorname{Reg} \cap\left[\aleph_{0}, \lambda\right]$ we speak on models of $T$, i.e. $\delta \in E, \operatorname{cf}(\delta)=\kappa$ whereas here we deal with pairs of models. However, to analyze $M_{\delta}$ for $\delta \in E \wedge \operatorname{cf}(\delta)=\kappa, E$ small enough club of $\lambda^{+}$, it is natural to assume $\delta=\sup \{\alpha \in E: \operatorname{cf}(\alpha)=\lambda$ and $\alpha<\delta\}$ and choose $\bar{\alpha} \in \operatorname{Seq}_{E, \kappa, \delta}$ which means $\bar{\alpha}$ is an increasing continuous sequence $\left\langle\alpha_{i}: i\langle\kappa\rangle\right.$ of ordinals with limit $\delta$ such that $i<\kappa$ non-limit $\Rightarrow \operatorname{cf}\left(\alpha_{i}\right)=\lambda$. So a sufficient condition for $\mathbf{n}_{\lambda, \kappa}(T)=1$ is $\mathbf{n}_{\lambda, \kappa}^{*}(T)=1$ where $\mathbf{n}_{\lambda, \kappa}^{*}=\operatorname{Min}_{E}\left|\left\{M_{\bar{\alpha}} / \cong: \bar{\alpha} \in \operatorname{Seq}_{E, \kappa, \delta}\right\}\right|, E$ varying on the clubs of $\lambda^{+}$. Now though it is not clear if this is also a necessary condition it seems more approachable and is natural. Anyhow it seems reasonable to consider $\mathbf{n}_{\lambda, 2}^{*}(T)=1$, i.e. the generic pair conjecture.

This connects us to the long term goal of classifying first order theories by "good" dividing lines, ones in which we find outside properties (like here investigating $\mathbf{n}_{\lambda, \kappa}(T)$ or just $\mathbf{n}_{\lambda, \lambda}(T)$, trying to characterize it) with "inside" definitions (like being dependent), and developing an inside theory; here - looking at decomposition (in $\S 1$ decompositions of models, in $\S 2$ decomposition of types, in $\S 3, \S 4$ strict decomposition of types). More fully, for this we have to analyze types. In $\S 1$ we make a first attempt; more exactly see 1.8 and 1.9. We try to analyze a model $N:=M_{\beta}$ over $M:=M_{\alpha}$ by trying to find models $M_{1}, N_{1}$ such that:

$$
\boxplus_{1} M_{\alpha}=M \prec M_{1} \prec N_{1} \text { and } M_{\alpha}=M \prec N=M_{\beta} \prec N_{1}
$$


$\boxplus_{2}$ for every $\bar{a} \in \omega>\left(M_{1}\right)$ for some $B_{\alpha} \in[M]^{<\lambda}$ the type $\operatorname{tp}\left(\bar{a}, M_{\beta}, M_{1}\right)$ is definable over $B_{\alpha}$ in a weak sense, i.e. does not split over $B_{\alpha}$, this means that if $n<\omega$ and $\bar{b}, \bar{c} \in{ }^{n}\left(M_{\alpha}\right)$ realizes the same type over $B_{\alpha}$ then so does $\bar{a}^{\wedge} \bar{b}, \bar{a}^{\wedge} \bar{c}$ (this is parallel to $(*)_{8.1}$ from $\odot_{8}$ ); it follows that for any sequence $\bar{a} \in{ }^{\kappa>}\left(M_{1}\right)$ a similar statement holds

$\boxplus_{3} \operatorname{tp}\left(N_{1}, M_{1}, N_{1}\right)$ is weakly orthogonal to every $q \in \mathbf{S}^{<\omega}\left(M_{1}\right)$ which does not split over some $B \in\left[M_{1}\right]^{<\lambda}$; the weakly orthogonal means that $q$ has a unique extension in $\mathbf{S}^{n}\left(N_{1}\right)$ wherever $q \in \mathbf{S}^{n}\left(M_{1}\right)$.

In $\S 2$ we try to analyze a type rather than a pair of models, also we find it better to deal with $\theta$-types, $\theta \geq|T|$, as during the analysis we add more variables. So for a $\kappa$-saturated model $M \prec \mathfrak{C}$ and sequence $\bar{d}$ of length $<\theta^{+}$we try to analyze $\operatorname{tp}(\bar{d}, M, \mathfrak{C})$ in two steps. The first is to add $\bar{c}$ of length $<\theta^{+}$such that

$\boxplus_{4} \operatorname{tp}(\bar{c}, M, \mathfrak{C})$ does not split over some $B \subseteq M \prec \mathfrak{C}$ of cardinality $<\kappa$.

This corresponds to the stable type ("unfortunately" but unavoidably depending on $\kappa)$, so for the theory of dense linear orders it corresponds to types $p \in \mathbf{S}(M)$ with $\mu_{p}<\kappa$, see $(*)_{8.1}$ above. True, they are not really definable, but non-splitting is a weak form of being definable. The second step is

$\boxplus_{5} \operatorname{tp}(\bar{d}, M+\bar{c}, \mathfrak{C})$ is tree like, i.e. if $A \subseteq M \prec \mathfrak{C}$ and $|A|<\kappa \underline{\text { then for some }}$ $\bar{e} \in \theta^{\theta^{+}>} M$ we have $\operatorname{tp}(\bar{d}, \bar{c}+\bar{e}) \vdash \operatorname{tp}(\bar{d}, A+c)$.

This property holds for $T=\operatorname{Th}(\mathbb{Q},<), p \in \mathbf{S}(M)$ when $\mu_{p} \geq \kappa$ !, i.e. when both cofinalities are $\geq \kappa$. This is the Type Decomposition Theorem (2.4).

A consequence is some clarification of models of $M$ of a dependent theory which are exactly $\kappa$-saturated for singular $\kappa$. We deal with this question to some extent per se.

In $\S 3$ we get a better decomposition - strict decomposition. But at present with a price, assuming e.g. $\kappa=\|M\|$ is a measurable cardinal. The main point appears in $\S 4$, the existence of limits of increasing sequences of strict decompositions.

Using this we are able to prove the pair genericity conjecture, the structure side for the case of a measurable cardinal. The measurability assumption seems undesirable. Describing this to Udi Hrushovski he was more concerned about also having the non-structure side for independent $T$. Now at the time in [Sh:877] it was remarked that a similar proof should work for the strongly inaccessibles, but the author was not motivated enough to really look into it. Subsequently [Sh:906] completes it.

The order of the sections is by their conceptions, so there are some repetitions. In Sh:950 and Kaplan-Shelah KpSh:946 we start to continue this work as well as in Sh:F1127]. Note that [Sh:950 concentrate on saturated models but it works just as well for special models (in singular strong limit cardinals, see e.g. CK73).

We thank the referee with thoroughness much above the call of duty causing the paper to be much improved and John Baldwin for much helpful criticism.

Context 0.1. 1) $T$ is complete first order theory.

2) $\mathfrak{C}=\mathfrak{C}_{T}$ is a monster model for $T$, omitting $T$ when no confusion arises; i.e. $\bar{\kappa}$ is a large enough cardinal, $\mathfrak{C}$ is a $\bar{\kappa}$-saturated model such that we deal only with models $M \prec \mathfrak{C}$, sets $A \subseteq \mathfrak{C}$ of cardinality $<\bar{\kappa}$ and sequences $\bar{a}, \bar{b}, \bar{c}, \bar{d}, \bar{e}$ from ${ }^{\alpha} \mathfrak{C}$ for some $\alpha<\bar{\kappa}$. So $\operatorname{tp}(\bar{c}, A)$ means $\operatorname{tp}(\bar{c}, A, \mathfrak{C})$. 
3) We may not pedantically distinguish a model $M$ and its universe, the cardinality $\|M\|$ of $M$ is that of its universe.

Notation 0.2.1) For $M \prec \mathfrak{C}$ and $\bar{a} \in{ }^{\alpha} M$ or just $\bar{a} \in{ }^{\alpha} \mathfrak{C}$ let $M_{[\bar{a}]}$ be the expansion of $M$ by every relation $R_{\varphi(\bar{x}, \bar{a})}=\varphi(M, \bar{a})$ where $\varphi(M, \bar{a}):=\left\{\bar{b} \in{ }^{\ell g(\bar{x})} M: \mathfrak{C} \models \varphi[\bar{b}, \bar{a}]\right\}$ for $\varphi(\bar{x}, \bar{y}) \in \mathbb{L}\left(\tau_{T}\right)$ such that $\ell g(\bar{y})=\alpha, \ell g(\bar{x})<\omega$ or pedantically $\varphi(\bar{x}, \bar{y}\lceil u)$ for $\bar{x}, \bar{y}$ as above, $u \subseteq \alpha$ finite. We define $M_{[A]}$ similarly, i.e. as the expansion of $M$ by $R_{\varphi(\bar{x}, \bar{a})}=\varphi(M, \bar{a})$ for every $\in^{\ell g(\bar{y})} A$ and $\varphi(\bar{x}, \bar{y}) \in \mathbb{L}\left(\tau_{T}\right)$.

$1 \mathrm{~A})$ For $p(\bar{x}) \in \mathbf{S}^{\alpha}(M)$ let $M_{[p]}$ be $M_{[\bar{a}]}$ whenever $\bar{a} \in{ }^{\alpha} \mathfrak{C}$ realizes $p(\bar{x})$.

1B) We say the sequence $\left\langle\varphi_{s}\left(\bar{x}, \bar{a}_{s}\right): s \in I\right\rangle$ of formulas from $\mathbb{L}\left(\tau_{M}\right)$ with $\bar{a}_{s}$ from $M$ is independent in the model $M$ when every finite non-trivial Boolean combination of sets from $\varphi_{s}\left(M, \bar{a}_{s}\right)$ is non-empty.

2) Writing $\varphi(\bar{x}, \bar{y}) \in \mathbb{L}\left(\tau_{T}\right), \varphi$ here is always first order but $\bar{x}$ and $\bar{y}$ may be infinite, though sometimes are finite (said or clear from the context). Let $p(\bar{x}), q(\bar{x}), r(\bar{x})$ denote types over some $A \subseteq \mathfrak{C}$, i.e. set of formulas of the form $\varphi(\bar{x}, \bar{b}), \bar{b} \in{ }^{(\ell g(\bar{b}))} A$. 3 ) $\mathrm{EC}_{\lambda}(T)$ is the class of models $M$ of $T$ (so $M \prec \mathfrak{C}$ ) of cardinality $\lambda$ and $\mathrm{EC}_{\lambda, \kappa}(T)$ is the class of $\kappa$-saturated $M \in \mathrm{EC}_{\lambda}(T)$.

4) $A+\bar{c}$ is $A \cup \operatorname{Rang}(\bar{c})$, etc.

$5)$ Let $\operatorname{tp}(A, B)$ be $\operatorname{tp}(\bar{a}, B)$ where $\bar{a}$ is the identity function on $A$.

Definition 0.3. 1) If $\bar{a}_{t} \in{ }^{\gamma} \mathfrak{C}$ for $t \in I$ and $\mathscr{D}$ is a filter on $I$ and $\bar{x}=\left\langle x_{i}: i<\gamma\right\rangle$ and $A \subseteq \mathfrak{C}$ then $\operatorname{Av}\left(\left\langle\bar{a}_{t}: t \in I\right\rangle / \mathscr{D}, A\right)=\left\{\varphi(\bar{x}, \bar{b}): \bar{b} \in{ }^{\omega>} A\right.$ and the set $\{t \in I:$ $\left.\mathfrak{C} \models \varphi\left[\bar{a}_{t}, \bar{b}\right]\right\}$ belongs to $\left.\mathscr{D}\right\}$. Note that if $T$ is dependent, $I$ is a linear order with no last members and $\left\langle\bar{a}_{t}: t \in I\right\rangle$ is an indiscernible sequence, see below then the result $\in \mathbf{S}^{\gamma}(A)$. Also note that if $\mathscr{D}$ is an ultrafilter on $I$ then $\operatorname{Av}\left(\left\langle\bar{a}_{t}: t \in \overline{I\rangle / \mathscr{D}}, A\right)\right.$ belongs to $\mathbf{S}^{\gamma}(A)$.

1A) Recall that if $\mathscr{D}$ is a filter on $\left\{\bar{a}_{t}: t \in I\right\} \subseteq{ }^{\alpha} \mathfrak{C}$ and $A \subseteq \mathfrak{C}$ we define $\operatorname{Av}(\mathscr{D}, A)$ similarly and if $I$ a linear order and $\mathscr{D}$ is the filter of co-bounded subsets of $I$ we may omit it.

2) If $p(\bar{x}), q(\bar{y})$ are complete types over $A$ we say $p(\bar{x}), q(\bar{y})$ are weakly orthogonal when for every $\bar{a}_{1}, \bar{a}_{2}$ realizing $p(\bar{x})$ and $\bar{b}_{1}, \bar{b}_{2}$ realizing $q(\bar{y})$ we have $\operatorname{tp}\left(\bar{a}_{1}{ }^{\wedge} \bar{b}_{1}, A\right)=$ $\operatorname{tp}\left(\bar{a}_{2}{ }^{\wedge} \bar{b}_{2}, A\right)$.

3) For a linear order $I,\left\langle\bar{a}_{s}: s \in I\right\rangle$ is an indiscernible sequence over $B$ when: $\ell g\left(\bar{a}_{s}\right)$ is constant and if $s_{0}<_{I} \ldots<_{I} s_{n-1}$ an $t_{0}<_{I} \ldots<_{I} t_{n-1}$ then the sequences $\bar{a}_{s_{0}}{ }^{\wedge} \ldots{ }^{\wedge} \bar{a}_{s_{n-1}}$ and $\bar{a}_{t_{0}}{ }^{\wedge} \ldots{ }^{\wedge} \bar{a}_{t_{n-1}}$ realize the same type over $B$.

Recall also (see [Sh:c, Ch.II,, 4$]$ )

Fact 0.4. If $T$ is dependent then for any formula $\varphi=\varphi(\bar{x}, \bar{y}, \bar{z}) \in \mathbb{L}\left(\tau_{T}\right)$ there is $n=n_{\varphi}<\omega$ (depending on $T$ ) such that:

(a) for no $\bar{c} \in \ell g(\bar{z}) \mathfrak{C}$ and $\bar{b}_{i} \in \ell g(\bar{y}) \mathfrak{C}$ for $i<n$, is the sequence $\left\langle\varphi\left(\bar{x}, \bar{b}_{i}, \bar{c}\right)\right.$ : $i<n\rangle$ independent, i.e. every non-trivial Boolean combination of the sets $\varphi\left(M, \bar{b}_{i}, \bar{c}\right)=\left\{\bar{a} \in{ }^{\ell g(\bar{x})} M: M \models \varphi\left[\bar{a}, \bar{b}_{i}, \bar{c}\right]\right\}$ for $i<n$ is non-empty.

(b) If $\left\langle\bar{b}_{i}: i<n\right\rangle$ is an indiscernible sequence over $C, \ell g\left(\bar{b}_{i}\right)=\ell g(\bar{y}), \bar{c} \in{ }^{\ell g(\bar{z})} C$ (all in $\mathfrak{C}$ ) then for no $\bar{a} \in{ }^{\ell g(\bar{a})} M$ do we have $\mathfrak{C} \models \varphi\left[\bar{a}, \bar{b}_{i}, \bar{c}\right]^{\mathrm{if}(\ell \text { even })}$ for $\ell<n$.

(c) Also there is a finite $\Delta_{\varphi} \subseteq \mathbb{L}\left(\tau_{T}\right)$ such that in clause (b) it is enough to demand that $\left\langle\bar{b}_{i}: i<n\right\rangle$ is a $\Delta$-indiscernible sequence.

Lastly, we quote Erdös-Rado ER69. 
Fact 0.5. The $\Delta$-System Lemma for finite sets.

For every natural numbers $k, n$ there is a natural number $m$ such that: if $u_{i}$ is a finite set with $\leq k$ elements for $i<m$ then there are sets $w \subseteq\{0, \ldots, m-1\}$ with $|w|=n$ and $u_{*}$ such that $\left\langle u_{i}: i \in w\right\rangle$ is a $\Delta$-system with heart $u_{*}$, which means that $i \neq j \in w \Rightarrow u_{i} \cap u_{j}=u_{*}$.

Definition 0.6. 1) A partial order $I$ is $\kappa$-directed when every set $J \subseteq I$ of cardinality $<\kappa$ has an upper bound $t \in I$ which means that $(\forall s)\left(s \in J \Rightarrow s \leq_{I} t\right)$.

2) A sequence $\left\langle A_{s}: s \in I\right\rangle$ is an independent sequence of subsets of $A_{*}$ when $\left(A_{s} \subseteq A_{*}\right.$ for $s \in I$ and $) \bigcap_{s \in u} A_{s} \backslash \bigcup_{t \in v} A_{t}$ is non-empty for every disjoint finite $u, v \subseteq I$. 


\section{$\S 1$. Non-Splitting COnstructions}

On such constructions including $\mathbf{F}_{\kappa}^{\text {nsp }}$ see [Sh:C, Ch.IV, $\left., 1, \S 3\right]$ but $\mathbf{F}^{\text {nsp }}$ here is $\mathbf{F}^{p}$ there; and see [Sh:715, 4.23-4.26], however this section is self-contained.

We try here to analyze $\kappa$-saturated models $M \prec N$, e.g. by finding $M_{1}, N_{1}$ such that $M \prec M_{1} \prec N_{1}, N \prec N_{1}$ where $M_{1}$ is $\mathbf{F}_{\kappa}^{\text {nsp }}$-constructible over $M$, see below and $\operatorname{tp}\left(N_{1}, M_{1}\right)$ is weakly orthogonal to any type over $M_{1}$ realized in some $\mathbf{F}_{\kappa}^{\mathrm{nsp}}$-construction over it, see Theorem[1.9] part (B) noting that $M, N, N_{1}, M_{1}$ here stands for $A, A^{+}, M, N$ there. We first recall the definition of non-splitting and some of its properties.

Definition 1.1. We say $p(\bar{x})$ does not split over $A$ when : if $\varphi(\bar{x}, \bar{b}), \neg \varphi(\bar{x}, \bar{c}) \in p(\bar{x})$ then $\operatorname{tp}(\bar{b}, A) \neq \operatorname{tp}(\bar{c}, A)$.

Fact 1.2. 1) If $\circledast_{A, B, C}$ below holds and $p(\bar{x}) \in \mathbf{S}^{m}(B)$ does not split over $A$, then there is one and only one $q(\bar{x}) \in \mathbf{S}^{m}(C)$ extending $p(\bar{x})$ and not splitting over $A$ (also called the non-splitting extension of $p(\bar{x})$ over $C$ ), where:

$\circledast_{A, B, C}(a) \quad A \subseteq B \subseteq C$

(b) for every $\bar{c} \in{ }^{\omega>} C$ there is $\bar{b} \in{ }^{\ell g(\bar{c})} B$ realizing $\operatorname{tp}(\bar{c}, A)$.

2) Let $I$ be a linear order. If $\operatorname{tp}\left(\bar{a}_{t}, B \cup \bigcup\left\{\bar{a}_{s}: s<_{I} t\right\}\right)$ does not split over $B$ and increases with $t \in I$ then $\left\langle\bar{a}_{t}: t \in I\right\rangle$ is an indiscernible sequence over $B$.

3) If $\operatorname{tp}(\bar{a}, B)$ does not split over $A$, the sequence $\left\langle\bar{b}_{t}: t \in I\right\rangle$ is an indiscernible sequence over $A$ and $\bar{b}_{t} \subseteq B$ for $t \in I$ then $\left\langle\bar{b}_{t}: t \in I\right\rangle$ is an indiscernible sequence over $A \cup \bar{a}$.

4) If $A \subseteq B$ then the number of $p \in \mathbf{S}^{\theta}(B)$ which does not split over $A$ is $\leq$ $2^{2^{|A|+|T|+\theta}}$, moreover if $T$ is dependent the number is $\leq 2^{|A|+|T|+\theta}$.

5) If $A \subseteq B$ and $p \in \mathbf{S}^{\alpha}(B)$ is finitely satisfiable in $A$ then $p$ does not split over $A$.

Proof. 1) By [Sh:3] or see [Sh:715] or see [Sh:300a, 1.10] for uniqueness.

2) By [Sh:c, I] or [Sh:300, I] or [Sh:300a, 3.2].

3) By the definitions.

4) The first conclusion is easy and see [Sh:3] or [Sh:300a, §1], the second holds by Sh:783, 5.26].

5) Easy, too.

Fact 1.3. [Assume $T$ is dependent.]

If $p(\bar{x})$ is an $\alpha$-type over $B \subseteq A$ then we can find $q(\bar{x}) \in \mathbf{S}^{\alpha}(A)$ extending $p(\bar{x})$ such that for some $C \subseteq A$ of cardinality $\leq|T|+|\alpha|$ the type $q(\bar{x})$ does not split over $B \cup C$.

Proof. [Sh:c, III,7.5,pg.140] or see [Sh:715, 4.24].

Observation 1.4. For $\kappa$ regular.

1) If $A \subseteq B,|A|<\kappa$ and $\bar{a} \in \kappa>\mathfrak{C}$ and $\operatorname{tp}(\bar{a}, B)$ is finitely satisfiable in $A$ then it does not split over $A$.

2) If $A \subseteq B, \bar{c} \in \kappa>\mathfrak{C}$ and $\operatorname{tp}(\bar{c}, B)$ does not split over $A$ and $i<\ell g(\bar{c})$ then $\operatorname{tp}\left(c_{i}, B \cup\left\{c_{j}: j<i\right\}\right)$ does not split over $A \cup\left\{c_{j}: j<i\right\}$. Similarly for $\left\langle\bar{c}_{j}: j \overline{<i\rangle}\right.$ when $j \leq i \Rightarrow \operatorname{Rang}\left(\bar{c}_{j}\right) \subseteq \operatorname{Rang}(\bar{c})$.

3) If $\operatorname{tp}\left(\bar{c}_{k}, B+\bar{c}_{0}+\ldots+\bar{c}_{k-1}\right)$ does not split over $A \subseteq B$ for $k<n$ then $\operatorname{tp}\left(\bar{c}_{0}{ }^{\wedge} \ldots{ }^{\wedge} \bar{c}_{n-1}, B\right)$ does not split over $A$. 
4) If $A \subseteq A_{1} \subseteq B_{1} \subseteq B$ and $\operatorname{Rang}\left(\bar{c}_{1}\right) \subseteq \operatorname{Rang}(\bar{c})$ and $\operatorname{tp}(\bar{c}, B)$ does not split over $A$ then $\operatorname{tp}\left(\bar{c}_{1}, B_{1}\right)$ does not split over $A_{1}$.

5) If $\bar{c} \in{ }^{\kappa>} \mathfrak{C}$ and for every finite $u \subseteq \ell g(\bar{c})$ and finite $B_{1} \subseteq B$ the type $\operatorname{tp}\left(\bar{c}\left\lceil u, B_{1}\right)\right.$ does not split over $A$ then $\operatorname{tp}(\bar{c}, B)$ does not split over $A$.

Proof. Easy.

As in [Sh:C, IV].

Definition 1.5. 1) $\mathscr{A}$ is an $\mathbf{F}_{\kappa}^{\mathrm{nsp}}$-construction when:

(a) $\mathscr{A}=(A, \bar{a}, \bar{B}, \bar{A}, \alpha)=\left(A^{\mathscr{A}}, \bar{a}^{\mathscr{A}}, \bar{B}^{\mathscr{A}}, \bar{A}^{\mathscr{A}}, \alpha^{\mathscr{A}}\right)$,

(b) $\bar{a}=\left\langle a_{\beta}: \beta<\alpha\right\rangle=\left\langle a_{\beta}^{\mathscr{A}}: \beta<\alpha\right\rangle$,

(c) $\bar{B}=\left\langle B_{\beta}: \beta<\alpha\right\rangle=\left\langle B_{\beta}^{\mathscr{A}}: \beta<\alpha\right\rangle$,

(d) $\bar{A}=\left\langle A_{\beta}: \beta \leq \alpha\right\rangle=\left\langle A_{\beta}^{\mathscr{A}}: \beta \leq \alpha\right\rangle$,

(e) $A_{\beta}=A \cup\left\{a_{\gamma}: \gamma<\beta\right\}$,

(f) $B_{\beta} \subseteq A_{\beta}$ and $\left|B_{\beta}\right|<\kappa$,

(g) $\operatorname{tp}\left(a_{\beta}, A_{\beta}\right)$ does not split over $B_{\beta}$.

2) We let $\ell g(\mathscr{A})=\alpha^{\mathscr{A}}$ and writing $\mathscr{A}$ we may omit $\bar{A}^{\mathscr{A}}, \alpha^{\mathscr{A}}$ as they are determined by the others so may write $\mathscr{A}=(A, \bar{a}, \bar{B})$ or $\mathscr{A}=\left(A,\left\langle\left(a_{\beta}, B_{\beta}\right): \beta<\alpha\right\rangle\right)$. We may replace $a_{\beta}$ by a finite sequence $\bar{a}_{\beta}$ with no real change.

3) We say the $\mathbf{F}_{\kappa}^{\text {nsp }}$-construction $\mathscr{A}$ is $\mu$-full when $\operatorname{cf}(\ell g(\mathscr{A})) \geq \kappa$ and if $q \in$ $\mathbf{S}\left(A_{\ell g(\mathscr{A})}^{\mathscr{A}}\right)$ does not split over $B$ where $B \subseteq \overline{A_{\ell g(\mathscr{A})}^{\mathscr{A}}}$ has cardinality $<\kappa$, then $\left\{\beta<\ell g(\mathscr{A}): a_{\beta}\right.$ realizes $p\left\lceil A_{\beta}^{\mathscr{A}}\right.$ and $\left.B \subseteq A_{\beta}^{\mathscr{A}}\right\}$ is unbounded in $\alpha^{\mathscr{A}}$ and has order type divisible by $\mu$.

4) We say $C$ is $\mathbf{F}_{\kappa}^{\mathrm{nsp}}$-constructible over $A$ when there is an $\mathbf{F}_{\kappa}^{\mathrm{nsp}}$-construction $\mathscr{A}$ such that $A=A^{\mathscr{A}}=A_{0}^{\mathscr{A}}$ and $C=A_{\ell g(\mathscr{A})}^{\mathscr{A}}$.

Definition 1.6. 1) Let $A \leq_{\kappa} C$ mean that $C$ is $\mathbf{F}_{\kappa}^{\text {nsp }}$-constructible over $A$.

2) We say that $\left(A^{+}, A\right)$ is $\kappa$-reduced when: if $A \leq_{\kappa} C$ and $\bar{c} \in{ }^{\kappa>}\left(A^{+}\right)$then $\operatorname{tp}(\bar{c}, A)$ has a unique extension to a complete type over $C$.

$3)$ We say the $(N, M)$ is $\kappa$-nice when:

(a) $(N, M)$ is $\kappa$-reduced and $M \prec N$,

(b) $M$ is $\kappa$-saturated,

(c) $N$ is $\kappa$-saturated,

(d) if $M \leq_{\kappa} M^{+}$then $M_{[N]} \prec M_{[N]}^{+}$, see below.

3A) Recall $M_{[B]}$ is $M$ expanded by $R_{\varphi(\bar{x}, \bar{a})}=\left\{\bar{b} \in{ }^{\ell g(\bar{x})} M: \mathfrak{C} \models \varphi[\bar{b}, \bar{a}]\right\}$ for $\varphi(\bar{x}, \bar{y}) \in \mathbb{L}\left(\tau_{T}\right)$ (with $\bar{x}$ finite of course), $\bar{a} \in{ }^{\ell g(\bar{y})} N$ and recall $\operatorname{Th}\left(M_{[B]}\right)$ is dependent by [Sh:783, $\S 1]$.

4) We say that $(M, A)$ is pseudo $\kappa$-reduced when: if $\bar{c} \in{ }^{\omega>} A,\left\|M_{1}\right\|<\kappa, M_{1} \subseteq$ $M, q(\bar{x}) \in \mathbf{S}^{<\omega}(M)$ is finitely satisfiable in $M_{1}$ then $q(\bar{x}), \operatorname{tp}(\bar{c}, M)$ are weakly orthogonal.

Observation 1.7. For $\kappa$ regular:

1) $\leq_{\kappa}$ is a partial order.

2) If $\left\langle A_{i}: i \leq \alpha\right\rangle$ is increasing continuous and $i<\alpha \Rightarrow A_{i} \leq_{\kappa} A_{i+1}$ then $A_{0} \leq_{\kappa} A_{\alpha}$.

3) In Definition [1.6(2) it is enough to consider $\bar{c} \in{ }^{\omega>}(C \backslash A)$. 
4) If $A \leq_{\kappa} B$ and $\bar{c} \in{ }^{\kappa>} B$ then ${ }^{2} \operatorname{tp}(\bar{c}, A)$ does not split over some $A^{\prime} \subseteq A$ of cardinality $<\kappa$.

$5)$ If the pair $(M, C)$ is $\kappa$-reduced then $(M, C)$ is pseudo $\kappa$-reduced.

$6)$ If $\operatorname{tp}(\bar{a}, A)$ does not split over $B$ and $B \subseteq A$ has cardinality $<\kappa$ then $A \leq_{\kappa} A+\bar{a}$.

Proof. Easy; e.g. part (6) by 1.4(2) and part (4) by $1.3(3),(4))$ and part (5) by 1.2 .

Claim 1.8. [ $T$ is dependent and $\kappa=\operatorname{cf}(\kappa)>|T|]$.

1) For every $A$ there is a $\kappa$-saturated $C$ such that $A \leq_{\kappa} C$ and $|C| \leq(|A|+|T|)^{<\kappa}$.

2) If in addition $\mu \leq(|A|+|T|)^{<\kappa}$ then we can add " $C$ is " $\mu$-full $\kappa$-saturated"; clearer if $\left.|C| \leq(|A|+|T|)^{<\kappa}+2^{2^{\kappa}}\right)$.

Proof. 1) By $1.3+1.7(2)$ and 1.7(6).

2) Similarly (by 1.2(4)).

Now we arrive to the first result giving a decomposition. The type $\operatorname{tp}\left(A^{+}, A\right)$ is decomposed in Theorem 1.9 by finding $M$ such that $A \leq_{\kappa} M$, (so the complete types over $A$ realized in $M$ are somewhat definable) and $\left(A^{+}, M\right)$ is $\kappa$-reduced, so the type $\operatorname{tp}\left(A^{+}, M\right)$ is weakly orthogonal to types in $\mathbf{S}^{<\omega}(M)$ not splitting over subsets of $M$ of cardinality $<\kappa$.

Theorem 1.9. The Density of Reduced Pairs Theorem [T dependent].

For any $A \subseteq \overline{A^{+} \text {and } \kappa=\operatorname{cf}(\kappa)>|T| \text { and } \lambda \text { satisfying }}$ 3 $\theta<\kappa \Rightarrow \lambda=\lambda^{\theta} \geq\left|A^{+}\right|$

(A) we can find $M$ such that $M$ is a model of cardinality $\lambda$ such that $A \leq_{\kappa} M$ and $\left(A^{+}, M\right)$ is $\kappa$-reduced

(B) for some $M$ as in clause (A) and $N$ the pair $(N, M)$ is $\kappa$-reduced and even $\kappa$-nice and $A^{+} \subseteq N$.

Proof. Proof of (A):

Our intention is to try to do a construction as described in $\nabla$ below. Having carried the induction the proof is divided to two cases. In the first we get the desired conclusion. In the second, we get a contradiction to $T$ being dependent; formally to the maximality of the $k$ chosen in $(g)(\beta)$ of $\otimes$.

We choose $M_{i}, B_{i}, j_{i}, \bar{c}_{i}$ by induction on $i \leq \lambda^{+}$such that

$\otimes(a) \quad M_{i}$ is $\prec$-increasing continuous, $M_{i}$ of cardinality $\leq \lambda+|i|$,

(b) $j_{i} \leq i, B_{i} \prec M_{i},\left\|B_{i}\right\|<\kappa$,

(c) $M_{0}$ is $\mathbf{F}_{\kappa}^{\mathrm{nsp}}$-constructible over $A$,

(d) $\quad M_{i+1}$ is $\mathbf{F}_{\kappa}^{\text {nsp }}$-constructible over $M_{i}$ and $M_{i+1}$ is $\kappa$-saturated,

(e) $\bar{c}_{i} \in{ }^{\omega>}\left(M_{i+1}\right)$ and $B_{i} \subseteq M_{j_{i}}$ has cardinality $<\kappa$,

(f) $\operatorname{tp}\left(\bar{c}_{i}, M_{i}\right)$ does not split over $B_{i}$,

$(g) \quad$ if $\operatorname{cf}(i) \geq \kappa$ and subclause $(\alpha)$ below holds then subclause $(\beta)$ holds where:

Subclause $(\alpha)$ : There are $j<i, m<\omega, B \subseteq M_{j}$ of cardinality $<\kappa$ and $\overline{p(\bar{x}) \in \mathbf{S}^{m}\left(M_{i}\right)}$ which does not split over $B$ and $p(\bar{x})$ has $\geq 2$ extensions in $\mathbf{S}^{m}\left(M_{i} \cup A^{+}\right)$.

\footnotetext{
${ }^{2}$ we could have chosen this as the definition. This changes the places we really need " $\kappa$ regular".

${ }^{3}$ no real harm if we replace " $\theta<\lambda \Rightarrow \lambda=\lambda^{\theta} \geq\left|A^{+}\right|$" by $\theta<\kappa \Rightarrow \lambda=\lambda^{\theta}+2^{2^{\theta+|T|}} \geq\left|A^{+}\right|$ and then we can use only the first version of $1.2(4)$.
} 
Subclause $(\beta)$ : There are $m=m_{i}<\omega, j=j_{i}<i, k=k_{i}<\omega$ and

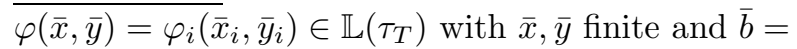
$\bar{b}_{i} \in{ }^{(\ell g(\bar{y}))}\left(M_{j} \cup A^{+}\right)$and $\varepsilon_{0}<\ldots<\varepsilon_{k-1}$ from the interval $\left[j_{i}, i\right)$ such that:

- $B_{i} \subseteq M_{j}$,

- $\operatorname{tp}\left(\bar{c}_{i}, M_{i}\right) \cup\left\{\varphi_{i}\left(\bar{x}, \bar{b}_{i}\right)\right\}$ and $\operatorname{tp}\left(\bar{c}_{i}, M_{i}\right) \cup\left\{\neg \varphi_{i}\left(\bar{x}, \bar{b}_{i}\right)\right\}$ are consistent,

- $\operatorname{tp}\left(\bar{c}_{\varepsilon_{\ell}}, M_{\varepsilon_{\ell}}\right)=\operatorname{tp}\left(\bar{c}_{i}, M_{\varepsilon_{\ell}}\right)$,

- $\mathfrak{C} \models \varphi\left[\bar{c}_{\varepsilon_{\ell}}, \bar{b}\right]^{\mathrm{if}(\ell \text { even })}$,

- $k$ is maximal for the given $\varphi(\bar{x}, \bar{y}), \bar{b}, j_{i}$ (see $\circledast$ below; $k$ is well defined as $T$ is dependent, see $\circledast$ below),

- $\mathfrak{C} \models \varphi\left[\bar{c}_{i}, \bar{b}\right]^{\mathrm{if}(k \text { is even })}$.

So in stage $i$ we first choose $M_{i}$ : if $i=0$ by clause (c), such model $M_{i}$ exists by 1.8(1), if $i$ is a limit ordinal we choose $M_{i}$ as $\cup\left\{M_{j}: j<i\right\}$ and if $i=j+1$ (so $\bar{c}_{j}$ has already been defined) then choose $M_{i}$ such that $M_{j} \cup \bar{c}_{j} \leq_{\kappa} M_{j+1}$ and $M_{i}=M_{j+1}$ is $\kappa$-saturated of cardinality $\lambda$ (and $\lambda$-full if you like), possible by Claim 1.8(1). Note that $M_{j} \leq_{\kappa} M_{j} \cup \bar{c}_{j}$ by clause (f) and 1.7(6) hence $M_{j} \leq_{\kappa} M_{j+1}$ recalling $1.7(2)$.

Note

$\circledast$ there is $n=n_{\varphi(\bar{x}, \bar{y})}$ depending on $\varphi(\bar{x}, \bar{y})$ and $T$ only such that in subclause $(\beta)$ we have $\varphi_{i}\left(\bar{x}_{i}, \bar{y}_{i}\right)=\varphi(\bar{x}, \bar{y}) \Rightarrow k_{i} \leq n$.

[Why? As by clause (f) in subclause $(\mathrm{g})(\beta)$ of $\boxplus(f)$, by $1.2(2)$ the sequence $\left\langle\bar{c}_{\varepsilon_{\ell}}\right.$ : $\ell<k\rangle$ is an indiscernible sequence, so by $T$ being dependent we are done by 0.4 .]

Second, why can we choose $\left(m_{i}, j_{i}, B_{i}, \varphi_{i}, \operatorname{tp}\left(\bar{c}_{i}, M_{i}\right)\right)$ as required in clause (g)? If $\operatorname{cf}(i)<\kappa$ or the antecedent of clause $(\mathrm{g})$, i.e. $(g)(\alpha)$ fails then trivially yes (choose e.g. $\bar{c}_{i}$ as the empty sequence). Otherwise let $j<i, B \subseteq M_{j}$ be of cardinality $<\kappa, m<\omega$ and $p(\bar{x}) \in \mathbf{S}^{m}\left(M_{i}\right)$ which does not split over $B$ and which has extensions $p_{0}(\bar{x}) \neq p_{1}(\bar{x})$ in $\mathbf{S}^{m}\left(M_{i} \cup A^{+}\right)$with $p_{0} \uparrow\left(M_{i} \cup A^{+}\right) \neq p_{1} \uparrow\left(M_{i} \cup A^{+}\right)$, so $p_{0}\left\lceil M_{i}=p=p_{1}\left\lceil M_{i}\right.\right.$ does not split over $B$.

Hence for some $\bar{b} \in{ }^{\omega>}\left(M_{i} \cup A^{+}\right)$and $\varphi=\varphi(\bar{x}, \bar{y}) \in \mathbb{L}\left(\tau_{T}\right)$ we have $\varphi(\bar{x}, \bar{b}) \in$ $p_{1}(\bar{x}), \neg \varphi(\bar{x}, \bar{b}) \in p_{0}(\bar{x})$; as $i$ is a limit ordinal without loss of generality $\bar{b} \in \omega>\left(M_{j} \cup\right.$ $\left.A^{+}\right)$. We now try to choose $\varepsilon_{\ell}$ by induction on $\ell \leq n_{\varphi(\bar{x}, \bar{y})}$ such that:

$\odot$ (a) $j \leq \varepsilon_{\ell}<i$ and $k<\ell \Rightarrow \varepsilon_{k}<\varepsilon_{\ell}$,

(b) $\bar{c}_{\varepsilon_{\ell}}$ realizes $p(\bar{x})\left\lceil M_{\varepsilon_{\ell}}\right.$,

(c) $\mathfrak{C} \models \varphi\left[\bar{c}_{\varepsilon_{\ell}}, \bar{b}\right]^{\mathrm{if}(\ell \text { is even })}$,

(d) $\varepsilon_{\ell}$ is minimal under $(\mathrm{a})+(\mathrm{b})+(\mathrm{c})$.

So by $\circledast$ for some $k \leq n_{\varphi(\bar{x}, \bar{y})}$ we have: $\varepsilon_{\ell}$ is well defined iff $\ell<k$. At last we choose:

$(*) \quad(a) \quad B_{i}=B$

(b) $\varphi_{i}=\varphi$

(c) $j_{i}=j$

(d) $\quad k_{i}=k$

(e) $\quad \bar{c}_{i}$ realizes $p_{1}(\bar{x})$ if $k$ is even and realizes $p_{0}(\bar{x})$ if $k$ is odd. 
So at last we are in a situation where the construction from $\otimes(a)-(g)$ having been carried out. So now comes the division to cases.

Let $S_{*}=\left\{i<\lambda^{+}: \operatorname{cf}(i) \geq \kappa\right.$ and subclause $(\alpha)$ of clause (g) holds for $\left.i\right\}$.

Recall that $S$ is a stationary subset of an ordinal $\delta$ of cofinality $>\aleph_{0}$ (e.g. a regular uncountable cardinal) when it is not disjoint to any closed unbounded subset $E$ of $\delta$.

Case 1: $S_{*}$ is a stationary subset of $\lambda^{+}$.

Hence for $i \in S_{*}$, there are $j_{i}, B_{i}, \varphi_{i}\left(\bar{x}_{i}, \bar{y}_{i}\right), \bar{b}_{i}$ and $k_{i}<\omega$ and $\varepsilon_{0}(i)<\ldots<$ $\varepsilon_{k_{i}-1}(i)<i$ as in subclause $(\beta)$ of $\otimes(g)$ and by Fodor's lemma (see e.g. [Jec03]) for some $m_{*}<\omega, j<\lambda^{+}, B, \varphi(\bar{x}, \bar{b}), k_{*},\left\langle\varepsilon_{i}: i<k^{*}\right\rangle$ and a stationary subset $S$ of $S_{*}=\left\{\delta<\lambda^{+}: \operatorname{cf}(\delta) \geq \kappa\right\}$ we have $\delta \in S \Rightarrow j_{\delta}=j \wedge B_{\delta}=B \wedge \ell g\left(\bar{c}_{\delta}\right)=$ $m_{*} \wedge \varphi_{\delta}\left(\bar{x}, \bar{b}_{\delta}\right)=\varphi(\bar{x}, \bar{b}) \wedge k_{\delta}=k_{*} \wedge \bigwedge_{\ell<k_{*}} \varepsilon_{\ell}(\delta)=\varepsilon_{\ell}$. Also without loss of generality by 1.2(4) we have $\delta \in S \Rightarrow \operatorname{tp}\left(\bar{c}_{\delta}, M_{\min (S)}\right)=\operatorname{tp}\left(\bar{c}_{\min (S)}, M_{\min (S)}\right)$ recalling that the number of such types is $\leq 2^{|B|+|T|}$. Choose $\delta(0)<\delta(1)$ from $S$ so both has cofinality $\geq \kappa$ and $B_{\delta(0)}=B_{\delta(1)}, \operatorname{tp}\left(\bar{c}_{\delta(0)}, M_{\delta(0)}\right) \subseteq \operatorname{tp}\left(\bar{c}_{\delta(1)}, M_{\delta(1)}\right)$ by 1.2(1) and $\bar{b}_{\delta(1)}=\bar{b}_{\delta(0)}$ and $\varepsilon_{i}(\delta(0))=\varepsilon_{i}(\delta(1))$ for $i<k_{*}$. But we could have chosen in stage $\delta(1), \varepsilon_{k}$ for $k<k_{*}$ and $k_{\delta(1)}^{\prime}=k_{*}+1$ and $\varepsilon_{k_{*}}^{\prime}(\delta(1))=\delta(0)$, contradiction to the maximality of $k$ in Subclause $(\beta)$ of $\otimes(g)$.

Case 2: Not Case 1 .

Then for a club of $i<\lambda^{+}$if $\operatorname{cf}(i) \geq \kappa$ then subclause $(\alpha)$ of Clause (g) fails for $i$ hence $M_{i}$ exemplifies that we have gotten the desired conclusion in (A) of 1.9 .

Proof of (B): By induction on $i<\lambda^{+}$we choose $M_{i}, M_{i}^{+}, B_{i}, j_{i}, \bar{c}_{i}$ such that

$\otimes^{\prime}$ Clauses (a),(c),(d) of $\otimes$ and

(h) $\left\langle M_{j}^{+}: j \leq i\right\rangle$ is $\prec$-increasing continuous and $A^{+} \subseteq M_{0}^{+}$,

(i) $\quad M_{i} \prec M_{i}^{+}$and $M_{i}^{+}$has cardinality $\lambda$ and if $i$ is non-limit then $M_{i}, M_{i}^{+}$are $\kappa$-saturated,

(j) if $\operatorname{cf}(i) \geq \kappa$ and there are $j_{i}<i, m<\omega, B \prec M_{j_{i}}$ of cardinality $<\kappa$ and $p \in \mathbf{S}^{m}\left(M_{i}\right)$ which does not split over $B$ and has $\geq 2$ extensions in $\mathbf{S}^{m}\left(M_{j}^{+}\right) \underline{\text { then }}$ subclause $(\beta)$ of clause $\otimes(g)$ above holds (with $\bar{b} \in{ }^{\ell g(\bar{y})}\left(M_{j}^{+}\right)$).

The rest of the proof is similar to that of (A). Alternatively, we choose $M_{i}, M_{i}^{+}$by induction on $i \leq \kappa$ such that Clauses (a),(c),(d) of $\nabla$ and (h),(i) of $\nabla^{\prime}$ and

(k) if $i=j+1$ then $\left(M_{j}^{+}, M_{i+1}\right)$ is $\kappa$-reduced.

Then $\left(M_{\kappa}^{+}, M_{\kappa}\right)$ are as required on $(N, M)$.

For the rest of the section we shall assume (as we use it all the time).

Hypothesis 1.10. $T$ is dependent. 
Definition 1.11. 1) $\mathbf{S}_{<\kappa}^{\mathrm{nsp}}(A)=\{p \in \mathbf{S}(A): p$ does not split over some $B \subseteq A$ of cardinality $<\kappa\}$.

2) $\mathbf{S}_{<\kappa}^{\mathrm{nsp}, \alpha}(A) \subseteq \mathbf{S}^{\alpha}(A)$ and $\mathbf{S}_{<\kappa}^{\mathrm{nsp},<\alpha}(A) \subseteq \bigcup_{\beta<\alpha} \mathbf{S}^{\beta}(A)$ are defined similarly.

3) $\mathbf{S}_{\geq \kappa}^{\mathrm{nsp}}(A)=\left\{p \in \mathbf{S}(A): p\right.$ is weakly orthogonal to $r$ for every $\left.r \in \mathbf{S}_{<\kappa}^{\mathrm{nsp}}(A)\right\}$.

4) $\mathbf{S}_{\geq \kappa}^{\mathrm{nsp}, \alpha}(A), \mathbf{S}_{\geq \kappa}^{\mathrm{nsp},<\alpha}(A)$ are defined similarly.

We may note

Observation 1.12. 1) If $\operatorname{tp}\left(\bar{c}_{1}, A\right)$ belongs to $\mathbf{S}_{\geq \kappa}^{\mathrm{nsp}, \alpha}(A)$ and $\bar{c}_{2} \in{ }^{\beta} \mathfrak{C}$ and $\operatorname{Rang}\left(\bar{c}_{2}\right) \subseteq$ $\operatorname{Rang}\left(\bar{c}_{1}\right)$ then $\operatorname{tp}\left(\bar{c}_{2}, A\right)$ belongs to $\mathbf{S}_{\geq \kappa}^{\mathrm{nsp}, \beta}$.

2) $\operatorname{tp}(\bar{c}, A) \in \mathbf{S}_{\geq \kappa}^{\text {nsp, } \alpha}(A) \underline{\text { iff }} \operatorname{tp}\left(\bar{c}\lceil u, A) \in \mathbf{S}_{\geq \kappa}^{\text {nsp }, m}(A)\right.$ for every finite $u \subseteq \ell g(\bar{c})$.

3) If $\operatorname{tp}(\bar{a}, A) \in \mathbf{S}^{m}(A)$ is weakly orthogonal to $\operatorname{tp}(\bar{c}, A)$ and does not split over $B \subseteq A$ and every $q \in \mathbf{S}^{<\omega}(B)$ is realized in $M$ then $\operatorname{tp}(\bar{a}, A+\bar{c})$ does not split over $B$.

Proof. Straight.

Observation 1.13. If $\kappa=\operatorname{cf}(\kappa)>|T|$, the model $M$ is $\kappa$-saturated and $p \in$ $\mathbf{S}^{m}(M)$, then we can find $N, q$ such that:

$$
\begin{aligned}
& \circledast_{N}^{1} \text { (a) }\|N\|=\|M\|<, \\
&(b) \quad q \in \mathbf{S}^{m}(N) \text { extends } p, \\
&(c) \quad N \text { is } \mathbf{F}_{<\kappa}^{\text {nsp }} \text {-constructible over } M, \\
& \circledast_{N, q, \kappa}^{2} \text { (a) } N \text { is } \kappa \text {-saturated and } q \in \mathbf{S}^{m}(N) . \\
& \\
& \text { (b) } \quad \text { if } r \in \mathbf{S}_{<\kappa}^{\mathrm{nsp},<\omega}(N) \text { then } r, q \text { are weakly orthogonal, i.e. } q \in \mathbf{S}_{\geq \kappa}^{\mathrm{nsp}, m}(N) .
\end{aligned}
$$

Proof. Let $\bar{c}$ realize $p(\bar{x})$ and let $C=\operatorname{Rang}(\bar{c})$, now we apply clause (A) of Theorem 1.9 with $M, M \cup C, N$ here standing for $A, A^{+}, M$ there.

Theorem 1.14. The Tree-like Type Theorem Assume $q(\bar{x}) \in \mathbf{S}_{\geq_{\kappa}}^{\mathrm{nsp}, \alpha}(N)$ and $N$ is $\kappa$-saturated and $\kappa>\theta=|T|+|\alpha|$ and let $\bar{z}=\left\langle z_{\alpha}: \alpha<\theta\right\rangle$. Then we can find a sequence $\bar{\psi}=\left\langle\psi_{\varphi(\bar{x}, \bar{y})}(\bar{x}, \bar{z}): \varphi(\bar{x}, \bar{y}) \in \mathbb{L}\left(\tau_{T}\right)\right\rangle$ of formulas such that for every $A \subseteq M$ of cardinality $<\kappa$ there is $\bar{c} \in{ }^{\theta} M$ such that:

(a) $\left\{\psi_{\varphi(\bar{x}, \bar{y})}(\bar{x}, \bar{c}): \varphi(\bar{x}, \bar{y}) \in \mathbb{L}\left(\tau_{T}\right)\right\} \subseteq q \nmid \operatorname{Rang}(\bar{c}) \subseteq q$,

(b) for each $\varphi(\bar{x}, \bar{y}) \in \mathbb{L}\left(\tau_{T}\right)$ we have $\psi_{\varphi(\bar{x}, \bar{y})}(\bar{x}, \bar{c}) \vdash\left\{\varphi(\bar{x}, \bar{b}): \bar{b} \in{ }^{\ell g(\bar{y})} A\right.$ and $\varphi(\bar{x}, \bar{b}) \in q\}$.

Proof. This follows from Claims 1.15, 1.16 below.

Claim 1.15. 1) Assume that $\circledast_{N, q, \kappa}^{2}$ from the Claim 1.13 holds, which means $N$ is $\kappa$-saturated and $q \in \mathbf{S}_{\geq \kappa}^{\mathrm{nsp}}(N)$. Then

$\circledast_{N, q, \kappa}^{3}$ if $M \prec N$ has cardinality $<\kappa$ and $\varphi(x, y)$ is a formula with parameters from $N$, then for some $\psi(x, \bar{d})=\psi_{\varphi(x, y), M}\left(x, \bar{d}_{\varphi(x, y), M}\right) \in q$ and $\eta \in{ }^{M} 2$ we have $\psi(x, \bar{d}) \vdash p_{\varphi(x, y)}^{M, \eta}$ where

$$
p_{\varphi(x, y)}^{M, \eta}=\left\{\varphi(x, b)^{\eta(b)}: b \in M\right\} ; \text { so it is included in } q .
$$

2) Part (1) works also for $q \in \mathbf{S}^{m}(N)$, i.e. $q \in \mathbf{S}_{\geq \kappa}^{\mathrm{nsp}, m}(N)$ and $\varphi=\varphi(\bar{x}, \bar{y})$ where $\ell g(\bar{x})=m, \ell g(\bar{y})<\omega$. 
Proof. Fix $M$ such that $M \prec N$ of cardinality $<\kappa$.

1) First note that

$(*)_{1}$ if $D$ is an ultrafilter on $M$ then $q(x)$ is weakly orthogonal to $r_{D}=\operatorname{Av}(D, N)$.

[Why? As $r_{D}$ does not split over $M$, by 1.4(1).]

Second, note that

$(*)_{2}$ the following type cannot be finitely satisfiable in $M$ :

$$
\begin{aligned}
r^{*}(y)= & \left\{\left(\exists x_{1}\right)\left(\psi\left(x_{1}, \bar{d}\right) \wedge \varphi\left(x_{1}, y\right)\right): \psi(x, \bar{d}) \in q\right\} \cup \\
& \left\{\left(\exists x_{2}\right)\left(\psi\left(x_{2}, \bar{d}\right) \wedge \neg \varphi\left(x_{2}, y\right)\right): \psi(x, \bar{d}) \in q\right\} .
\end{aligned}
$$

[Why? Otherwise for some ultrafilter $D$ on $M$ we have $\vartheta(y, \bar{d}) \in r^{*}(y) \Rightarrow \vartheta(M, \bar{d}) \in$

$D$. Let $b \in \mathfrak{C}$ realize $\operatorname{Av}(D, N)$ so as $q(\bar{x})$ is closed under conjunctions, $q(x) \cup$ $\{\varphi(x, b)\}$ and $q(x) \cup\{\neg \varphi(x, b)\}$ are finitely satisfiable in $\mathfrak{C}$, and we get a contradiction to $(*)_{1}$.]

$(*)_{3}$ there is $\psi(x, \bar{d}) \in q$ such that $\left\{\left(\exists x_{1}\right)\left(\psi\left(x_{1}, \bar{d}\right) \wedge \varphi\left(x_{1}, y\right)\right),\left(\exists x_{2}\right)\left(\psi\left(x_{2}, \bar{d}\right) \wedge\right.\right.$ $\left.\left.\neg \varphi\left(x_{2}, y\right)\right)\right\}$ is satisfied by no $b \in M$.

[Why? By the monotonicity in $\psi(x, \bar{d})$ and $q$ being closed under conjunctions this follows from $(*)_{2}$.]

$(*)_{4}$ let $\psi_{\varphi(x, y), M}\left(x, \bar{d}_{\varphi(x, y), M}\right)=\psi(x, \bar{d})$, from $(*)_{3}$,

$(*)_{5}$ for every $b \in M$ we have $N \models$ " $(\forall x)(\psi(x, \bar{d}) \rightarrow \varphi(x, b))$ " or $N \models "(\forall x)(\psi(x, \bar{d}) \rightarrow$ $\neg \varphi(x, \bar{b})) "$

[Why? By logic this follows by $(*)_{3}$.]

$(*)_{6}$ there is $\eta \in{ }^{M} 2$ such that for every $b \in M$ we have $M \models "(\exists x)(\psi(x, \bar{d}) \wedge$ $\varphi(x, b)) "$ iff $M \models " \neg(\exists x)(\psi(x, y)) \wedge \neg \varphi(x, b) "$ iff $\eta(b)=1$.

[Why? By $(*)_{5}+(*)_{3}$.]

So we are done.

2) Similarly.

Claim 1.16. 1) In the previous claim 1.15, fixing $p$, if $\operatorname{cf}(\kappa)>|T| \underline{\text { then }} \psi$ depends on $\varphi$ but does not depend on $M$ though $\bar{d}$ in general does, i.e. given $\varphi(\bar{x}, \bar{y})$ we may assume without loss of generality that $\psi_{\varphi}(\bar{x}, \bar{d})=\psi_{\varphi(\bar{x}, \bar{y})}\left(x, \bar{d}_{\varphi(\bar{x}, \bar{y}), M}\right)$.

2) Assume $\circledast_{N, q, \kappa}^{2}$ from claim 1.13, i.e. $N$ is $\kappa$-saturated and $q \in \mathbf{S}_{\geq \kappa}^{\mathrm{nsp}, m}(N)$. Then the following partial order is $\kappa$-directed

(a) elements: $q\lceil B$ for $B \subseteq N$ of cardinality $\leq|T|$

(b) order: $p_{1} \leq p_{2}$ if $p_{2} \vdash p_{1}$.

Proof. 1) As if $N_{1} \prec N_{2} \prec N$ and $\left\|N_{2}\right\|<\kappa$, then $\psi_{\varphi(\bar{x}, \bar{y}), N_{2}}\left(x, \bar{d}_{\varphi(\bar{x}, \bar{y}), N_{2}}\right)$ can serve as $\psi_{\varphi(\bar{x}, \bar{y}), N_{1}}\left(\bar{x}, \bar{d}_{\varphi(\bar{x}, q), N_{2}}\right)$.

2) Easy.

As a conclusion we can now show that a key fact in [Sh:877 for the theory $T=\operatorname{Th}(\mathbb{Q},<)$ has a parallel for every dependent $T$.

\footnotetext{
${ }^{4}$ from some form of strongly dependent we should be able to get "essentially finite"
} 
Conclusion 1.17. The Saturated Expansion Conclusion Assume

(a) $N$ is $\kappa$-saturated,

(b) $|A|<\kappa$,

(c) if $\bar{a} \in A$ then $q_{\bar{a}}=\operatorname{tp}(\bar{a}, N) \in \mathbf{S}_{\geq \kappa}^{\mathrm{nsp},<\omega}(N)$, see Definition 1.11(3),

(d) $N_{[A]}$ has elimination of quantifiers.

Then $N_{[A]}$ is א-saturated.

Remark 1.18. 1) Recall $N_{[A]}$ is $N$ expanded by $R_{\varphi(\bar{x}, \bar{b})}=\left\{\bar{a} \in{ }^{\ell g(\bar{x})} N: \mathfrak{C} \models \varphi[\bar{a}, \bar{b}]\right\}$ for $\varphi(\bar{x}, \bar{b})$ a formula with parameters from $A$, see [Sh:783, $\S 1]$.

2 ) We can omit assumption (d) in 1.17, but then get $\kappa$-saturated only for quantifier free types.

Proof. Without loss of generality $\kappa$ is regular, this as it is enough to prove $\lambda^{+}$saturation for every $\lambda \in[|A|, \kappa)$. Let $M \prec N$ be such that $\|M\|<\kappa$ and assume $p=p(\bar{y}) \in \mathbf{S}^{m}\left(|M|, N_{[A]}\right)$ and we shall prove that some $\bar{c} \in{ }^{m} N$ realizes $p(\bar{y})$. Actually without loss of generality $M_{[A]} \prec N_{[A]}$ and by assumption (d), equivalently $p(\bar{y}) \in \mathbf{S}^{m}(M \cup A)=\mathbf{S}^{m}(M \cup A, \mathfrak{C})$ is finitely satisfiable in $M$. Let $\overline{\mathbf{c}}=\left\langle c_{\alpha}: \alpha<\alpha^{*}\right\rangle$ list $A$ so $\alpha^{*}<\kappa$ and for $u \subseteq \alpha^{*}$ let $\bar{c}_{u}=\left\langle c_{\alpha}: \alpha \in u\right\rangle, \bar{x}_{u}=\left\langle x_{\alpha}: \alpha \in u\right\rangle$.

Next note that by Claims 1.16(1) and 1.15(2) (here clause (c) of the assumption is used) applied to $\operatorname{tp}\left(\bar{c}_{u}, N\right)$ noting $\bar{y}$ of length $m$ is fixed and letting $\bar{x}_{u}=\left\langle x_{\alpha}\right.$ : $\alpha \in u\rangle$, we have:

$(*)_{1}$ for every finite $u \subseteq \alpha^{*}$ and formula $\varphi=\varphi\left(\bar{x}_{u}, \bar{y}, \bar{z}\right) \in \mathbb{L}\left(\tau_{T}\right)$ there are $\psi_{\varphi\left(\bar{x}_{u}, \bar{y}, \bar{z}\right)}\left(\bar{x}_{u}, \bar{d}_{\varphi\left(\bar{x}_{u}, \bar{y}, \bar{z}\right), M}\right) \in \operatorname{tp}\left(\left\langle c_{\alpha}: \alpha \in u\right\rangle, N\right)$ so $\bar{d}_{\varphi\left(\bar{x}_{u}, \bar{y}, \bar{z}\right), M} \in \omega>N$ and $\eta$ a function from ${ }^{\ell g(\bar{y})+\ell g(\bar{z})} M$ to $\{0,1\}$ such that:

$$
\psi_{\varphi\left(\bar{x}_{u}, \bar{y}, \bar{z}\right)}\left(\bar{x}_{u}, \bar{d}_{\varphi\left(\bar{x}_{u}, \bar{y}, \bar{z}\right), M}\right) \vdash\left\{\varphi\left(\bar{x}_{u}, \bar{b}, \bar{c}\right)^{\eta\left(\bar{b}^{\wedge} \bar{c}\right)}: \bar{b} \in{ }^{\ell g(\bar{y})} M \text { and } \bar{c} \in{ }^{\ell g(\bar{z})} M\right\} .
$$

Clearly $|p(\bar{y})|<\kappa$ so there are $\zeta^{*}<\kappa$ and a sequence $\left\langle\left(\varphi_{\zeta}\left(\bar{x}_{u_{\zeta}}, \bar{y}, \bar{z}_{\zeta}\right), u_{\zeta}\right): \zeta<\zeta^{*}\right\rangle$ listing the pairs $\left(\varphi\left(\bar{x}_{u}, \bar{y}, \bar{z}\right), u\right)$ as above so we have

$$
p(\bar{y})=\left\{\varphi_{\zeta}\left(\overline{\mathbf{c}}\left\lceil u_{\zeta}, \bar{y}, \bar{e}\right): \zeta<\zeta^{*}<\kappa \text { and } \bar{e} \in{ }^{\ell g\left(\bar{z}_{\zeta}\right)} M\right\},\right.
$$

so $u_{\zeta} \subseteq \alpha^{*}$ is finite.

For each $\zeta<\zeta^{*}$ we choose $\psi_{\zeta}\left(\bar{x}_{u_{\zeta}}, \bar{d}_{\zeta}\right)$ as guaranteed by $(*)_{1}$ above $\left(\right.$ for $\left.\varphi_{\zeta}\left(\bar{x}_{u_{\zeta}}, \bar{y}, \bar{z}\right)\right)$.

Let

$$
p^{\prime}(\bar{y}):=\left\{\left(\forall \bar{x}_{u_{\zeta}}\right)\left[\psi_{\zeta}\left(\bar{x}_{u_{\zeta}}, \bar{d}_{\zeta}\right) \rightarrow \varphi_{\zeta}\left(\bar{x}_{u_{\zeta}}, \bar{y}, \bar{e}\right)\right]: \zeta<\zeta^{*} \text { and } \bar{e} \in{ }^{\ell g\left(\bar{z}_{\zeta}\right)} M\right\} .
$$

Now

$(*)_{2} p^{\prime}(\bar{y})$ is finitely satisfiable in $M$.

[Why? As $p(\bar{y})$ finitely satisfiable in $M$, using the translation and the choice of $\psi_{\zeta}\left(\bar{x}_{u_{\zeta}}, \bar{d}_{\zeta}\right)$. That is, let $p^{\prime \prime}(\bar{y})$ be a finite subset of $p^{\prime}(\bar{y})$ so for some $k<\omega, \zeta_{\ell}=$ $\zeta(\ell)<\zeta^{*}, \bar{c}_{\ell} \in{ }^{\ell g\left(\bar{z}_{\zeta}\right)} M$ for $\ell<k$ we have $p^{\prime \prime}(\bar{y})=\left\{\left(\forall \bar{x}_{u_{\zeta(\ell)}}\left[\psi_{\zeta(\ell)}\left(\bar{x}_{u_{\zeta(\ell)}}, \bar{d}_{\zeta(\ell)}\right) \rightarrow\right.\right.\right.$ $\left.\left.\varphi_{\zeta}\left(\bar{x}_{u_{\zeta(\ell)},}, \bar{y}, \bar{e}_{\ell}\right)\right]: \ell<k\right\}$. Now $\left\{\varphi_{\zeta_{\ell}}\left(\overline{\mathbf{c}}\left\lceil u_{\zeta_{\ell}}, \bar{y}, \bar{e}_{\ell}\right): \ell<k\right\}\right.$ is a finite subset of $p(\bar{y})$ hence is realized by some $\bar{b} \in{ }^{m} M$, hence by $(*)_{1}$ the sequence $\bar{b}$ realizes $p^{\prime \prime}(\bar{y})$.] 
$(*)_{3}$ the type $p^{\prime}(\bar{y})$ is over $\cup\left\{\bar{d}_{\zeta}: \zeta<\zeta^{*}\right\} \cup M \subseteq N$.

[Why? Check.]

$(*)_{4} p^{\prime}(\bar{y})$ has cardinality $\leq|A|+|T|+\|M\|<\kappa$.

[Why? Obvious.]

$(*)_{5}$ there are $M^{+}$and $\bar{b}$ such that:

(a) $M \prec M^{+} \prec N$,

(b) $\operatorname{tp}\left(M^{+}, M \cup \bigcup\left\{\bar{d}_{\zeta}: \zeta<\zeta^{*}\right\}\right)$ is finitely satisfiable in $M$,

(c) $\bar{b} \in M^{+}$realizing $p^{\prime}(\bar{y})$.

[Why? Easy, e.g. using ultrapower, " $N$ is $\kappa$-saturated" and $(*)_{2}+(*)_{3}$.]

$(*)_{6} \quad \bar{b}$ realizes $p(\bar{y}) \in \mathbf{S}^{m}(N \cup A)$ and $\bar{b} \in{ }^{\ell g(\bar{y})}\left(M^{+}\right) \subseteq{ }^{\ell g(\bar{y})} N$.

[Why? Follow the translations.]

So we are done.

Question 1.19. 1) Can we waive assumption (d) in 1.17?

$2)$ Is the family of $(N, A)$ as in 1.17 "dense under $\leq_{\kappa}$ "?

Discussion 1.20. 1) Assume $\lambda=\lambda^{<\lambda}>\kappa=\operatorname{cf}(\kappa)>|T|$ and we try to prove that there is a $(\lambda, \kappa)$-limit model.

So let $M \in \mathrm{EC}_{\lambda}(T)$ be saturated and we try to analyze the class of $N, M \prec$ $N \in \mathrm{EC}_{\lambda}$, which are "close enough", in the sense of $(\lambda, \kappa)$-limit model.

So if $p \in \mathbf{S}_{<\lambda}^{\text {nsp }}(M)$, say $p$ does not split over $B$, for some $B \subseteq M$ of cardinality $<\lambda$, then we can assume that in $N$ there are "enough elements" realizing "types not-splitting over $B$ " extensions of $p$. So hopefully we can analyze such $N$ by $\mathscr{P} \subseteq \mathbf{S}_{\geq \lambda}^{\text {nsp }}(M)$ pairwise perpendicular or $\mathscr{P} \subseteq \mathbf{S}_{\geq \lambda}^{\text {nsp, }|T|}(M)$ such that for each $p \in \mathscr{P}$ the model $M_{[p]}$ from $0.2(1 \mathrm{~A})$ has elimination of quantifiers and is saturated, it is reasonable that this holds if we can expand $M$ by definition of $<\lambda$ types $p \in \mathscr{P}$.

What we need, i.e., what is necessary for this line of attack (but not yet clear if sufficient to carry it), is:

(*) if $\mathscr{P}_{\ell}$ above has cardinality $\lambda$ and is quite dense (e.g. using $\mathbf{F}$ 's for $\ell=1,2$ ) $\underline{\text { then }}$ there is an automorphism of $M$ which maps $\mathscr{P}_{1}$ onto $\mathscr{P}_{2}$.

This leads to the generic pair conjecture. About $\mathbf{S}_{<\lambda}^{\text {nsp }}(M)$ recall Definition 1.11(1). 


\section{$\S 2$. The TYPE DECOMPosition THEOREM}

Context 2.1. 1) $T$ is a complete first order theory; dependent if not said otherwise. 2) $\mathfrak{C}=\mathfrak{C}_{T}$ is a monster for $T$, etc. as in 0.1 .

Here we try to analyze a type $p \in \mathbf{S} \leq \theta(M)$ for $\kappa$-saturated $M \prec \mathfrak{C}$ where $\kappa>$ $\theta \geq|T|$, the characteristic case being $\kappa>>\theta\left(\theta\right.$ may be $\aleph_{0}$, if $T$ is countable). In the case of $\theta<|T|$, or even better $\theta<\aleph_{0}$ we know less but mention it. We look at " $T$ being stable" as our dream, our paradise. The hard reality is $T$ being just dependent. In some sense $T$ dependent should be like stable but we allow order, e.g. $\operatorname{Th}(\mathbb{Q},<)$ or trees. What we actually do is investigate the $K_{\ell}$ (see Definition 2.6).

How helpful is this analysis? We present two consequences. The first to some extent accomplished the professed aim: the Type Decomposition Theorem 2.4.

What is its meaning? If $M$ is $\kappa$-saturated, $\bar{d} \in \theta^{+}>\mathfrak{C}$ and $\kappa>\theta \geq|T|$ then we try to analyze the type $\operatorname{tp}(\bar{d}, M)$ in two steps: for some $\bar{c}, B$ :

(a) $B \subseteq M$ has cardinality $<\kappa$, say $B=|N|$,

(b) the type in the first step is similar to the types of stable theories, i.e. $\operatorname{tp}(\bar{c}, M)$ does not split over $B \subseteq M$; (we can even demand $\operatorname{tp}(\bar{c}, M)$ is finitely satisfiable in $B$ ),

(c) the type in the second step, $\operatorname{tp}(\bar{d}, M+\bar{c})$ behaves as in trees; e.g. letting $\mathbf{x}=(M, B, \overline{\mathbf{c}}, \mathbf{d})$ we have: on ${ }^{\theta} M$ the partial orders $\leq_{\mathbf{x}}$ is $\kappa$-directed (see (0.6)) where we let $\bar{a}_{1} \leq_{\mathrm{x}} \bar{a}_{2}$ iff $\operatorname{tp}\left(\bar{d}, \bar{c}^{\wedge} \bar{a}_{2}\right) \vdash \operatorname{tp}\left(\bar{d}, \bar{c}^{\wedge} \bar{a}_{1}\right)$.

The reader may say that Clause (b) is not a true parallel to a stable case, as $|B|$ is not bounded by $\theta+|T|$ (but this is impossible even for the theory of dense linear order). Still a type not splitting over a set is a weak form of definability. Also we may wonder, what is the meaning, when $T$ is $\operatorname{Th}(\mathbb{Q},<)$ ? If $M$ is $\kappa$-saturated each $p \in \mathbf{S}(M)$ actually stands for a cut of $M$. Now the cuts are divided to those which have cofinality $\geq \kappa$ from both sides (falling under (c)), and those which do not (hence fall under (b)).

The second consequence deals with singular $\mu$ of cofinality $>|T|$. We ask: is there $M \prec \mathfrak{C}$ which is exactly $\mu$-saturated, i.e. is $\mu$-saturated but not $\mu^{+}$-saturated. Now if $T=\operatorname{Th}(\mathbb{Q},<)$ this is impossible, that is, there is no such $M$. If $T$ is stable there is no problem to find such $M$, the main case being $\operatorname{cf}(\mu)>|T|$ (or just $\operatorname{cf}(\mu) \geq \kappa(T)$, see [Sh:c, Ch.III]) and let $M$ be $\mu$-prime over an indiscernible set of cardinality $\mu$. The result says that for dependent $T$ there is something like that, this is 2.2 ,

Lemma 2.2. Singular Exact Saturation Lemma Assume $\kappa$ is singular of cofinality

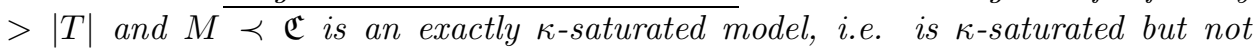
$\kappa^{+}$-saturated. Then there are $N$ and $A$ such that:

(a) $N \prec M$ of cardinality $<\kappa$ and $A \subseteq M$ of cardinality $\kappa$ and $M$ omits some $p \in \mathbf{S}(A)$ which does not split over $N$; in fact

(b) there is $q \in \mathbf{S}(M)$ which does not split over $N$ such that $p=q\lceil A$,

(c) there is an indiscernible sequence $\left\langle\bar{a}_{\alpha}: \alpha<\kappa\right\rangle$ over $N$ of $\theta$-tuples from $M$ such that $\operatorname{Av}\left(\left\langle\bar{a}_{\alpha}: \alpha<\kappa\right\rangle, N \cup\left\{\bar{a}_{\alpha}: \alpha<\kappa\right\}\right)$ is omitted by $M$ (equivalently, we cannot choose $\left.\bar{a}_{\kappa} \in M\right)$ and does not split over $N$. 
Remark 2.3. We can add in (c):

$(c)^{+}$moreover we can demand that there is an ultrafilter $\mathscr{D}$ on $N$ such that $M$ omits $p=\operatorname{Av}(\mathscr{D}, A)$ where $A:=\cup\left\{\bar{a}_{\alpha}: \alpha<\kappa\right\} \cup N$ and $p(\bar{x})$ is as in Clause (c).

Theorem 2.4. The Type Decomposition Theorem Assume $\operatorname{cf}(\kappa)>\theta \geq|T|, M$ is

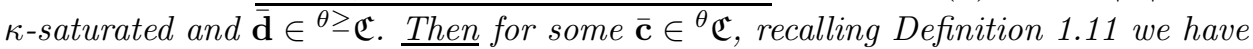
$\operatorname{tp}(\overline{\mathbf{c}}, M) \in \mathbf{S}_{<\kappa}^{\mathrm{nsp}, \theta}(M)$ and $\left(\mathbf{P}, \leq_{\mathbf{P}}\right)$ is a $\kappa$-directed partial order where:

(a) $\mathbf{P}=\{\operatorname{tp}(\overline{\mathbf{d}}, A \cup \overline{\mathbf{c}}): A \subseteq M$ has cardinality $\leq \theta\}$,

(b) $p_{1} \leq_{\mathbf{P}} p_{2}$ iff $p_{2} \vdash p_{1}$.

Remark 2.5. 1) In fact $\left(\mathbf{P}, \leq_{\mathbf{P}}\right)$ is $\left(\mathbf{P}_{\mathbf{x}, \theta}, \leq_{\mathbf{x}, \theta}\right)$ from the Definition 2.6 (8) below.

2) Note that being $\theta^{+}$-directed is obvious.

3) Would it be more transparent to use the notation $p_{2} \vdash p_{1}$ instead of $p_{1} \leq_{\mathbf{P}} p_{2}$ ? A matter of taste, the author feels that not.

Definition 2.6. 1) Let $K=K_{1}$ be the family of $\mathbf{x}$ satisfying

(a) $\mathbf{x}=(A, B, \overline{\mathbf{c}}, \overline{\mathbf{d}})$ but if $A=|M|$, as usual, we may write $M$ instead of $A$ and if $B=\emptyset$ we may omit it,

(b) $B \subseteq A$,

(c) $I$ a linear order,

(d) $\overline{\mathbf{c}}=\left\langle\bar{c}_{t, n}: n<n_{t}, t \in I\right\rangle$ where $n_{t} \leq \omega$, each $\bar{c}_{t, n}$ a finite 5 sequence and let 6 $\bar{c}_{t}=\bar{c}_{t, 0} \wedge \bar{c}_{t, 1} \wedge{ }^{\wedge} \bar{c}_{t, n_{t}-1}$

(e) $\left\langle\bar{c}_{t, n}: n<n_{t}\right\rangle$ is an indiscernible sequence over $A \cup\left\{\bar{c}_{s}: s \in I \backslash\{t\}\right\}$, so if $n_{t}=1$ this is an empty statement,

$(f)$ if $t \in I$ then $7 \operatorname{tp}\left(\bar{c}_{t},\left\{\bar{c}_{s}: s<_{I} t\right\} \cup A\right)$ does not split over $B$

$(g) \overline{\mathbf{d}}$ is a sequence of elements or finite sequences from $\mathfrak{C}$.

2) Let $K_{0}$ be defined similarly omitting clause $(f)$.

3) For $\lambda \geq \kappa, \operatorname{cf}(\lambda) \geq \theta$ (or just $\lambda \geq \theta$ ), $\operatorname{cf}(\kappa) \geq \theta$ and $\ell \in\{0,1\}$ let $K_{\lambda, \kappa,<\theta}^{\ell}=$ $\left\{(M, B, \overline{\mathbf{c}}, \overline{\mathbf{d}}) \in K_{\ell}: M\right.$ is $\lambda$-saturated, $|B|<\kappa$ and $\left.|\ell g(\bar{d})|+|\ell g(\overline{\mathbf{c}})| \mid<\theta\right\}$; omitting $\ell$ means 1. If $\theta=\sigma^{+}$instead of " $<\theta^{\prime}$ " we may write $\sigma$.

4)

(a) let $\mathbf{x}=\left(A_{\mathbf{x}}, B_{\mathbf{x}}, \overline{\mathbf{c}}_{\mathbf{x}}, \overline{\mathbf{d}}_{\mathbf{x}}\right)$ for $\mathbf{x} \in K_{0}$ (or $M_{\mathbf{x}}$ instead of $\left.A_{\mathbf{x}}\right), I=I_{\mathbf{x}}, \bar{c}_{\mathbf{x}, t}=$ $\bar{c}_{t}, n_{t}=n_{\mathbf{x}, t}$ and $C_{\mathbf{x}}=\cup\left\{\operatorname{Rang}\left(\bar{c}_{t, n}\right): t \in I\right.$ and $\left.n<n_{\mathbf{x}, t}\right\}$,

(b) we may 8 replace $\overline{\mathbf{d}}$ by $D_{\mathbf{x}}=\operatorname{Rang}(\overline{\mathbf{d}})$,

(c) we may omit $\kappa$ if $\kappa=\lambda$.

\footnotetext{
$5_{\text {we remark when it matters. }}$

${ }^{6}$ but abusing our notation, $\operatorname{Rang}(\overline{\mathbf{c}})$ is the set of elements of $\mathfrak{C}$ appearing in it; similarly in other cases

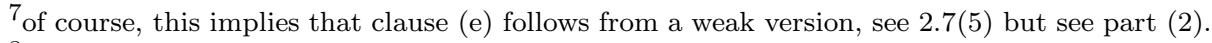

${ }^{8}$ we may write $C_{\mathbf{x}}=\operatorname{Rang}(\overline{\mathbf{c}}), \bar{c}_{\mathbf{x}, t, n}=\left\langle c_{t, n, m}^{\mathbf{x}}: m\left\langle\ell g\left(c_{\mathbf{x}, t, n}\right)\right\rangle\right.$ so in fact $\bar{c}_{\mathbf{x}}=\left\langle c_{t, n, m}^{\mathbf{x}}\right.$ : $(t, m, m) \in J\rangle$ for the natural $J=J_{\mathbf{x}}$.
} 
For $\lambda=\aleph_{0}$, let " $<\lambda$ " mean $A_{\mathbf{x}}$ is the universe of $M_{\mathbf{x}} \prec \mathfrak{C}$ (no saturation demand). 5) We define a two-place relation $\leq_{0}$ on $K_{0}: \mathbf{x} \leq_{0} \mathbf{y}$ iff $A_{\mathbf{x}} \subseteq A_{\mathbf{y}}, B_{\mathbf{x}} \subseteq B_{\mathbf{y}}, I_{\mathbf{x}} \subseteq$ $I_{\mathbf{y}}, \overline{\mathbf{c}}_{\mathbf{x}}=\overline{\mathbf{c}}_{\mathbf{y}}\left\lceil I_{\mathbf{x}}\right.$, i.e. $t \in I_{\mathbf{x}} \Rightarrow \bar{c}_{\mathbf{y}, t}=\bar{c}_{\mathbf{x}, t}$ moreover $t \in I_{\mathbf{x}} \Rightarrow n_{\mathbf{y}, t}=n_{\mathbf{x}, t}$ and $t \in I_{\mathbf{x}} \wedge n<n_{\mathbf{x}, t} \Rightarrow \bar{c}_{\mathbf{y}, t, n}=\bar{c}_{\mathbf{x}, t, n}, \overline{\mathbf{d}}_{\mathbf{x}} \unlhd \overline{\mathbf{d}}_{\mathbf{y}}$ and $\operatorname{tp}\left(\overline{\mathbf{c}}_{\mathbf{x}}, A_{\mathbf{y}}\right)$ does not split over $B_{\mathbf{x}}$ hence "tp $\left(\overline{\mathbf{c}}_{\mathbf{x}}, A_{\mathbf{x}}\right)$ does not split over $B_{\mathbf{x}}$ " follows.

$5 \mathrm{~A}) \mathbf{x} \leq_{1} \mathbf{y}$ mean $\mathbf{x} \leq_{0} \mathbf{y}$ and $A_{\mathbf{y}}=A_{\mathbf{x}}$.

6) We define a two-place relation $\leq_{2}$ on $K_{0}: \mathbf{x} \leq_{2} \mathbf{y}$ iff $\mathbf{x} \leq_{1} \mathbf{y} \wedge \overline{\mathbf{d}}_{\mathbf{x}}=\overline{\mathbf{d}}_{\mathbf{y}}$.

7) $\mathbf{x} \in K_{\lambda, \kappa, \theta}^{0}$ is called normal when $\operatorname{Rang}\left(\overline{\mathbf{c}}_{\mathbf{x}}\right) \subseteq \operatorname{Rang}\left(\overline{\mathbf{d}}_{\mathbf{x}}\right)$.

8) For $\mathbf{x} \in K$, let $\mathbf{P}_{\mathbf{x}, \theta}=\left(\mathbf{P}_{\mathbf{x}, \theta} \leq_{\mathbf{x}, \theta}\right)$ be defined by:

(a) $\mathbf{P}_{\mathbf{x}}=\left\{\operatorname{tp}\left(\overline{\mathbf{d}}_{\mathbf{x}}, A+\overline{\mathbf{c}}_{\mathbf{x}}\right): A \subseteq A_{\mathbf{x}}\right.$ has cardinality $\left.\leq \theta\right\}$,

(b) $\leq_{\mathbf{x}, \theta}$ is the following two-place relation on $\mathbf{P}_{\mathbf{x}, \theta}: p_{1}\left(\bar{x}_{\overline{\mathbf{d}}_{\mathbf{x}}}\right) \leq_{\mathbf{x}, \theta} p_{2}\left(\bar{x}_{\overline{\mathbf{d}}_{\mathbf{x}}}\right)$ iff $p_{2} \vdash p_{1}$

9) If $\theta=|T|+\left|\ell g\left(\overline{\mathbf{d}}_{\mathbf{x}}\right)\right|+\left|\ell g\left(\overline{\mathbf{c}}_{\mathbf{x}}\right)\right|$, i.e. we use " $<\theta^{+}$", we may omit it.

Claim 2.7. 1) $K_{1} \subseteq K_{0}$.

2) $\leq_{i}$ is a partial order on $K_{0}$ for $i=0,1,2$.

3) If $i \in\{0,1,2\},\left\langle\mathbf{x}_{\alpha}: \alpha<\delta\right\rangle$ is $\leq_{i}$-increasing in $K_{\lambda, \kappa, \theta}^{0}$ where $\delta$ is a limit ordinal, $\left[\operatorname{cf}(\delta) \geq \theta^{+} \Rightarrow \bigwedge_{j<\delta} \overline{\mathbf{c}}_{\mathbf{x}_{j}}=\overline{\mathbf{c}}_{\mathbf{x}_{0}}\right]$ and $\left[i \leq 1 \wedge \operatorname{cf}(\delta) \geq \theta^{+} \Rightarrow\left(\bigwedge_{\alpha<\delta} \overline{\mathbf{d}}_{\mathbf{x}_{\alpha}}=\overline{\mathbf{d}}_{\mathbf{x}_{0}}\right)\right],[i=$ $0 \Rightarrow \lambda \leq \operatorname{cf}(\delta)]$ and $\delta<\operatorname{cf}(\kappa) \vee\left(\bigwedge_{\alpha<\delta} B_{\mathbf{x}_{\alpha}}=B_{\mathbf{x}_{i}}\right)$, then it has $a \leq_{i}$-lub $\mathbf{x}_{\delta}:=$ $\cup\left\{\mathbf{x}_{\alpha}: \alpha<\delta\right\} \in K_{\lambda, \kappa, \theta}^{0}$ defined by $A_{\mathbf{x}}=\cup\left\{A_{\mathbf{x}_{\alpha}}: \alpha<\delta\right\}, B_{\mathbf{x}}=\cup\left\{B_{\mathbf{x}_{\alpha}}: \alpha<\right.$ $\delta\}, I_{\mathbf{x}}=\cup\left\{I_{\mathbf{x}_{\alpha}}: \alpha<\delta\right\}, \overline{\mathbf{c}}_{\mathbf{x}}=\cup\left\{\overline{\mathbf{c}}_{\mathbf{x}_{\alpha}}: \alpha<\delta\right\}$, i.e. $\bar{c}_{\mathbf{x}, t}=\bar{c}_{\mathbf{x}_{\alpha}, t}$ when $t \in I_{\mathbf{x}_{\alpha}}$ and $\overline{\mathbf{d}}_{\mathbf{x}}=\cup\left\{\overline{\mathbf{d}}_{\mathbf{x}_{\alpha}}: \alpha<\delta\right\}$.

3A) In part (3), if $\alpha<\delta \Rightarrow \mathbf{x}_{\alpha} \in K_{\lambda, \kappa, \theta}^{1}$ then $\mathbf{x}_{\delta} \in K_{\lambda, \kappa, \theta}^{1}$.

4) If $\overline{\mathbf{d}} \in \theta^{+}>\mathfrak{C}$ and $M$ is $\kappa$-saturated and $\left.\kappa>\theta \underline{\text { then }} \mathbf{x}=(M, \emptyset,<>, \overline{\mathbf{d}})\right) \in K_{\kappa, \theta}^{\ell}$ for $\ell=0,1$.

5) In the definition of $\mathbf{x} \in K_{1}$ : in clause (e) it suffices to demand that: if $n_{t}>1$ then $\left\langle\bar{c}_{t, n}: n<n_{t}\right\rangle$ is indiscernible over $A \cup\left\{\bar{c}_{s, m}: s<_{I} t, m<n_{s}\right\}$.

6) For every $\mathbf{x} \in K_{\lambda, \kappa, \theta}$ there is a normal $\mathbf{y} \in K_{\lambda, \kappa, \theta}$ satisfying $\mathbf{x} \leq_{1} \mathbf{y}, \overline{\mathbf{c}}_{\mathbf{x}}=\mathbf{c}_{\mathbf{y}}$ and $\operatorname{Rang}\left(\overline{\mathbf{d}}_{\mathbf{y}}\right)=\operatorname{Rang}\left(\overline{\mathbf{d}}_{\mathbf{x}}\right) \cup \operatorname{Rang}\left(\overline{\mathbf{c}}_{\mathbf{x}}\right)$. Hence $\mathbf{y} \in \operatorname{mxK}_{\lambda, \kappa, \theta} \Leftrightarrow \mathbf{x} \in \operatorname{mxK}_{\lambda, \kappa, \theta}$, see Definition 2.8 below.

7) If $i=0$ and $\left\langle\mathbf{x}_{\alpha}: \alpha<\delta\right\rangle$ is $\leq_{i}$-increasing in $K_{\lambda, \kappa, \theta}^{0}$ and $\operatorname{cf}(\delta)<\theta^{+}, \delta<\operatorname{cf}(\kappa)$, then the sequence has $a \leq_{i}$-upper bound $\mathbf{x}_{\delta} \in K_{\lambda, \kappa, \theta}^{0}$, note that we have not said "lub".

7A) In part (7), if $\alpha<\delta \Rightarrow \mathbf{x}_{\alpha} \in K_{\lambda, \kappa, \theta}^{1} \underline{\text { then }}$ we can add $\mathbf{x}_{\delta} \in K_{\lambda, \kappa, \theta}^{1}$.

Proof. Easy e.g.

7), 7A) The problem is when part (3) does not cover it, so $\lambda>\aleph_{0}$. It is clear how to choose $\overline{\mathbf{c}}_{\mathbf{x}_{\delta}}, \overline{\mathbf{d}}_{\mathbf{x}_{\delta}}$ and $B_{\mathbf{x}_{\delta}}$, but we should choose a $\lambda$-saturated $M_{\mathbf{x}_{\delta}}$.

Let $B=\cup\left\{B_{\mathbf{x}_{\alpha}}: \alpha<\delta\right\}, I=\cup\left\{I_{\mathbf{x}_{\delta}}: \alpha<\delta\right\}$ and $\overline{\mathbf{c}}=\left\langle\bar{c}_{t}: t \in I\right\rangle$ with $\bar{c}_{t}=\bar{c}_{\mathbf{x}_{\alpha}, t}$ when $\alpha \in I_{\mathbf{x}_{\alpha}} ;$ similarly $\overline{\mathbf{d}}$.

First, choose a $\lambda$-saturated $M$ extending $\cup\left\{M_{\mathbf{x}_{\alpha}}: \alpha<\delta\right\}$ but what about "tp $\left(\bar{c}_{\mathbf{x}_{\alpha}}, M\right)$ does not split over $B_{\mathbf{x}_{\alpha}}$ for each $\alpha<\delta " ?$

Now for each $\alpha<\gamma<\delta, \operatorname{tp}\left(\overline{\mathbf{c}}_{\mathbf{x}_{\alpha}}, M_{\gamma}\right)$ does not split over $B_{\mathbf{x}_{\alpha}}$ which means $p_{\alpha, \gamma}(\bar{x})=\operatorname{tp}\left(\overline{\mathbf{c}}_{\mathbf{x}_{\alpha}}, M_{\gamma}\right)$ does not split over $B_{\mathbf{x}_{\alpha}}$ hence $p_{\alpha}(\bar{x}):=\cup\left\{p_{\alpha, \beta}(\bar{x}): \beta \in\right.$

${ }^{9}$ This follows from " $\left\langle\mathbf{x}_{\alpha}: \alpha<\delta\right\rangle$ is $\leq_{i}$-increasing" when $i=2$. 
$(\alpha, \delta)\}=\operatorname{tp}\left(\overline{\mathbf{c}}_{\mathbf{x}_{\alpha}}, \bigcup_{\beta<\delta} M_{\beta}\right)$ does not split over $B_{\mathbf{x}_{\alpha}}$. Also for $\alpha<\delta$ by $1.2(1)$ there is $\overline{\mathbf{c}}_{\alpha}^{\prime}$ such that $\operatorname{tp}\left(\overline{\mathbf{c}}_{\alpha}^{\prime}, M\right)$ does not split over $B_{\mathbf{x}_{\alpha}}$ and extends $p_{\alpha}$. As $M_{\mathbf{x}_{\alpha}} \supseteq B_{\mathbf{x}_{\alpha}}$ is $\lambda$-saturated and $\lambda \geq \kappa$ by Definition 2.6. (3) clearly the model $M_{\mathbf{x}_{\beta}}$ is $\kappa$-saturated and $\left|B_{\mathbf{x}_{\alpha}}\right|<\kappa$ by the definition of $K_{\lambda, \kappa, \theta}^{i}$. Recalling $1.2(1)$, by the last two sentences $\overline{\mathbf{c}}_{\beta}^{\prime}\left\lceil I_{\mathbf{x}_{\alpha}}\right.$ realizes $\operatorname{tp}\left(\overline{\mathbf{c}}_{\alpha}^{\prime}, M\right)$ for $\alpha<\beta<\delta$ hence without loss of generality $\alpha<\beta<$ $\delta \Rightarrow \overline{\mathbf{c}}_{\beta}^{\prime}\left\lceil I_{\mathbf{x}_{\alpha}}=\overline{\mathbf{c}}_{\alpha}^{\prime}\right.$.

Hence there is an elementary mapping $f$ mapping with domain $\cup\left\{\overline{\mathbf{c}}_{\alpha}^{\prime}: \alpha<\delta\right\} \cup$ $M$, mapping $\overline{\mathbf{c}}_{\alpha}^{\prime}$ to $\overline{\mathbf{c}}_{\mathbf{x}_{\alpha}}$ for $\alpha<\delta$, and extending $\operatorname{id}_{\cup\left\{M_{\alpha}: \alpha<\delta\right\}}$. Now $M_{\mathbf{x}_{\delta}}:=f(M)$ will do, i.e. let $M_{\mathbf{y}}=M, B_{\mathbf{y}}=B, \overline{\mathbf{c}}_{\mathbf{y}}=\overline{\mathbf{c}}, \overline{\mathbf{d}}_{\mathbf{y}}=\overline{\mathbf{d}}$.

Definition 2.8. 1) For $\ell=0,1$ let $\operatorname{mxK}_{\lambda, \kappa,<\theta}^{\ell}$ be the family of $\mathbf{x} \in K_{\lambda, \kappa,<\theta}^{\ell}$ which are $\leq_{2}^{+}$-maximal in $K_{\lambda, \kappa,<\theta}^{\ell}$, i.e. for no $\mathbf{y}$ do we have $\mathbf{x}<_{2}^{+} \mathbf{y} \in K_{\lambda, \kappa,<\theta}^{\ell}$, see below; if $\ell=1$ we may omit it.

2) For $i=0,1,2$ let $\leq_{i}^{+}$be the following two-place relation on $K_{0}: \mathbf{x} \leq_{i}^{+} \mathbf{y} \underline{\text { iff }}$ $\mathbf{x} \leq_{i} \mathbf{y}$, see Definition [2.6 and if $\mathbf{x} \neq \mathbf{y}$ then for some $t \in I_{\mathbf{y}} \backslash I_{\mathbf{x}}$ satisfying $n_{\mathbf{x}, t} \geq 2$ we have: $\mathfrak{C} \models \varphi\left[\overline{\mathbf{d}}_{\mathbf{x}}, \bar{c}_{t, 1}, \bar{b}\right] \wedge \neg \varphi\left[\overline{\mathbf{d}}_{\mathbf{x}}, \bar{c}_{t, 0}, \bar{b}\right]$ for some $\varphi=\varphi_{t}(\bar{x}, \bar{y}, \bar{z}) \in \mathbb{L}\left(\tau_{T}\right)$ and $\bar{b} \subseteq A_{\mathbf{x}} \cup \bigcup\left\{\bar{c}_{\mathbf{y}, s}: s \in I_{\mathbf{y}} \backslash\{t\}\right\}$.

3) Again, if $\theta=\sigma^{+}$instead of " $<\theta$ ": we may write $\sigma$, and if $\kappa=\lambda$ we may omit $\lambda$.

4) Of course, $\mathbf{x}<_{i}^{+} \mathbf{y}$ means $\mathbf{x} \leq_{i}^{+} \mathbf{y} \wedge \mathbf{x} \neq \mathbf{y}$.

Observation 2.9. Let $i=0,1,2$.

1) For $\ell=0,1$ the two-place relation $\leq_{i}^{+}$is a partial order on $K^{\ell}$.

2) If $\mathbf{x}_{1} \leq_{i} \mathbf{x}_{2}<_{i}^{+} \mathbf{x}_{3} \leq_{i} \mathbf{x}_{4}$ then $\mathbf{x}_{1}<_{i}^{+} \mathbf{x}_{4}$.

3) If $\mathbf{x}<_{i}^{+} \mathbf{z}$ are from $K_{\lambda, \kappa,<\theta}^{\ell} \underline{\text { then }}$ there is $\mathbf{y} \in K_{\lambda, \kappa,<\theta}^{\ell}$ such that $\mathbf{x}<_{2}^{+} \mathbf{y} \leq_{i} \mathbf{z}$ and $\overline{\mathbf{d}}_{\mathbf{y}}=\overline{\mathbf{d}}_{\mathbf{x}}, I_{\mathbf{y}} \backslash I_{\mathbf{x}}$ is finite.

4) The parallel of $2.7(3),(3 \mathrm{~A})$ holds for $\mathrm{mxK}$.

Proof. E.g.

3) Let $t \in I_{\mathbf{z}} \backslash I_{\mathbf{x}}$ and $\varphi=\varphi(\bar{x}, \bar{y}, \bar{z}) \in \mathbb{L}\left(\tau_{T}\right)$ and $\bar{b} \subseteq A_{\mathbf{x}} \cup \bigcup\left\{\bar{c}_{\mathbf{y}, s}: s \in I_{\mathbf{y}} \backslash\{t\}\right\}$ be such that $\mathfrak{C} \models \varphi\left[\overline{\mathbf{d}}_{\mathbf{x}}, \bar{c}_{\mathbf{y}, t, 1}, \bar{b}\right] \wedge \neg \varphi\left[\overline{\mathbf{d}}_{\mathbf{x}}, \bar{c}_{\mathbf{y}, t, 0}, \bar{b}\right]$. We choose a finite $I \subseteq I_{\mathbf{y}} \backslash\{t\}$ such that $\bar{b} \subseteq \cup\left\{\bar{c}_{\mathbf{y}, s}: s \in I\right\} \cup A_{\mathbf{x}}$. Now define $\mathbf{y}$ by: $M_{\mathbf{y}}=M_{\mathbf{x}}, I_{\mathbf{y}}=I_{\mathbf{x}} \cup I \cup\{t\}, \overline{\mathbf{c}}_{\mathbf{y}}=$ $\bar{c}_{\mathbf{z}} \backslash I_{\mathbf{y}}, \bar{d}_{\mathbf{y}}=\overline{\mathbf{d}}_{\mathbf{x}}$ and $B_{\mathbf{y}}=B_{\mathbf{x}}$.

Now check.

The following claim may be good for digesting the meaning of $\mathrm{mxK}_{\lambda, \kappa, \theta}^{\ell}$.

Claim 2.10. The L.S.T. Claim for $\mathrm{mxK}$

If $\mathbf{x} \in \operatorname{mx} \overline{\mathrm{K}_{\kappa, \theta}}$ and $M=M_{\mathbf{x}} \underline{\text { then }}$ for some function $F$ with domain $[M]^{<\kappa}$ satisfying $F(A) \in[M]^{<2^{|A|+|T|}}$ for $A \in \operatorname{Dom}(F)$, we have: if $M_{1} \prec M$ is closed under $F$ and contains $B_{\mathbf{x}}$ then $\left(M_{1}, B_{\mathbf{x}}, \overline{\mathbf{c}}_{\mathbf{x}}, \overline{\mathbf{d}}_{\mathbf{x}}\right)$ belongs to $\mathrm{mxK}_{\kappa, \theta}^{\ell}$.

Remark 2.11. By 2.9(4), it suffices to consider $F$ with domain $[M]^{<\aleph_{0}}$.

Proof. We can choose $F(\emptyset) \in[M]^{1}$ and for notational transparency we fix a set $J$ of cardinality $\aleph_{0}$ disjoint to $I_{\mathbf{x}}$.

Note that for every $N \prec M$ satisfying $B_{\mathbf{x}} \subseteq N$ we have $\mathbf{x}_{N}:=\left(N, B_{\mathbf{x}}, \overline{\mathbf{c}}_{\mathbf{x}}, \overline{\mathbf{d}}_{\mathbf{x}}\right) \in$ $K_{\kappa, \theta}$; call such $N$ a candidate. So to choose $F$ let us analyze the cases $B_{\mathbf{x}} \subseteq N \prec M$ but $\mathbf{x}_{N} \notin \operatorname{mxK}_{\kappa, \theta}^{\ell}$. Considering Definition 2.8, it suffices by 2.9(3) to consider the case $\mathbf{x}_{N}<_{2}^{+} \mathbf{y}, I_{\mathbf{y}} \backslash I_{\mathbf{x}}$ is finite and is $\subseteq J, t_{*} \in I_{\mathbf{y}} \backslash I_{\mathbf{x}}, \varphi_{*}(\bar{x}, \bar{y}, \bar{z}), \bar{b}_{*}$ as there, we can ignore the possibility that also some other $t \in I_{\mathbf{y}} \backslash I_{\mathbf{x}} \backslash\left\{t_{*}\right\}$ works. 
We let $\bar{b}_{0}$ list $B_{\mathbf{y}}, I_{\mathbf{y}} \backslash I_{\mathbf{x}}=\left\{t_{0}, \ldots, t_{k-1}\right\}, t_{*}=t_{\ell(*)}, \ell(*)<k, m_{t}>\ell g\left(\bar{c}_{y, t, 0}\right), n_{t}=$ $n_{\mathbf{y}, t}$ for $t \in I_{\mathbf{y}}$. Also without loss of generality $\varphi=\varphi\left(\bar{x}, \bar{y} ; \bar{z}_{1}, \bar{z}_{2}\right), \ell g(\bar{y})=\ell g\left(\bar{c}_{\mathbf{y}, t_{*}, 0}\right), \ell g(\bar{x})=$ $\ell g\left(\overline{\mathbf{d}}_{\mathbf{x}}\right), \bar{b}=\bar{b}_{1}{ }^{\wedge} \bar{b}_{2}, \bar{b}_{1} \in{ }^{\omega>}\left(M_{\mathbf{y}}\right)={ }^{\omega>} N, \ell g\left(\bar{z}_{1}\right)=\ell g\left(\bar{b}_{1}\right)$ and abusing our notation, $\ell g\left(\bar{z}_{2}\right)=\ell g\left(\bar{b}_{2}\right)$ where $\bar{b}_{2}=\left\langle\bar{c}_{\mathbf{y}, t, k}: k<n_{\mathbf{y}, t}, t \in I_{\mathbf{y}}\right\rangle$ and $\bar{b}_{2,<t}=\left\langle c_{\mathbf{y}, s, k}:\right.$ $k<n_{t}, s<t$ so $\left.t \in I_{\mathbf{y}}\right\rangle$ and $\bar{b}_{2, \neq t}=\left\langle\bar{c}_{\mathbf{y}, s, k}: k<n_{s}, s \in I_{\mathbf{y}} \backslash\{t\}\right\rangle$, so $\mathfrak{C} \models$ $\varphi\left[\overline{\mathbf{d}}_{\mathbf{x}}, \bar{c}_{\mathbf{y}, t_{*}, 1}, \bar{b}_{1}, \bar{b}_{2, t_{*}}\right] \wedge \neg \varphi\left[\overline{\mathbf{d}}_{\mathbf{x}}, \bar{c}_{\mathbf{y}, t_{*}, 0}, \bar{b}_{1}, \bar{b}_{2, t_{*}}\right]$ and let $\ell g\left(\bar{z}_{t}^{*}\right)=\ell g\left(\bar{c}_{\mathbf{y}, t}\right), \ell g\left(\bar{z}_{t, \ell}\right)=$ $\ell g\left(\bar{c}_{\mathbf{y}, t, \ell}\right)$ so $\bar{z}_{t}^{*}=\bar{z}_{t, 0}{ }^{\wedge} \ldots{ }^{\wedge} \bar{z}_{t, n_{t}-1}$ and $\bar{z}_{2}=\left(\ldots, \bar{z}_{t}^{*}, \ldots\right)_{t \in I_{\mathbf{y}}}$.

All this information will be called a witness against the candidate $N$ and we denote it by $\mathbf{w}$.

Let $\mathbf{s}$ consist of the following pieces of information on the witness $\mathbf{w}$ and in this case we shall say that $\mathbf{w}$ materializes $\mathbf{s}$ and $\mathbf{s}$ is a case for $N$.

$\otimes(a) \quad I=I_{\mathbf{y}}$ and $\left\langle t_{\ell}: \ell<k\right\rangle$ (so we will write $\left.I_{\mathbf{s}}, t_{\mathbf{s}, \ell}\right)$ and $\ell(*$ ),

(b) $n_{t}, m_{t}$ for $t \in I$,

(c) $\varphi=\varphi\left(\bar{x}, \bar{y}, \bar{z}_{1}, \bar{z}_{2}\right)$ hence $\ell g\left(\bar{b}_{1}\right)=\ell g\left(\bar{z}_{1}\right)$,

(d) $\zeta(0)=\ell g\left(\bar{b}_{0}\right)$ but not $\bar{b}_{0}$ itself,

(e) $q_{0}=\operatorname{tp}\left(\bar{b}_{0}, \emptyset\right)$ and $q_{1}=\operatorname{tp}\left(\bar{b}_{0}{ }^{\wedge} \bar{b}_{1}{ }^{\wedge} \bar{b}_{2}, \emptyset\right)$, so from $q_{1}$ we know when $b_{2, \ell_{1}}=c_{t_{\ell}, n, \ell_{2}}$

(f) the scheme of non-splitting of $\operatorname{tp}\left(\bar{c}_{t}, M\right)$ for $t \in I_{\mathbf{y}}$ from clause $(f)$ of 2.6 (1), that is $\Xi_{t}=\left\{\left(\psi\left(\bar{z}_{\bar{c}_{\mathbf{y}, t}}, \bar{y}^{\prime}\right), q\left(\bar{y}^{\prime}, \bar{y}_{\bar{b}_{0}}\right)\right)\right.$ : for some $\bar{b} \in{ }^{\ell g\left(\bar{y}^{\prime}\right)} N$ we have $\mathfrak{C} \models \psi\left[\bar{c}_{\mathbf{y}, t}, \bar{b}\right]$ and $\left.q\left(\bar{y}^{\prime}, \bar{y}_{\bar{b}_{0}}\right)=\operatorname{tp}\left(\bar{b}^{\wedge} \bar{b}_{0}, \emptyset\right)\right\}$.

We shall write $I=I_{\mathbf{s}}, t_{*}=t_{*}(\mathbf{s}), \varphi=\varphi_{\mathbf{s}}\left(\bar{x}_{\mathbf{s}}, \overline{\mathbf{y}}, \bar{z}_{\mathbf{s}, 1}, \bar{z}_{\mathbf{s}, 2}\right), q_{\mathbf{s}, 0}=q_{0}$, etc. and let $r_{\mathbf{s}}=\operatorname{tp}\left(\overline{\mathbf{c}}_{\mathbf{y}}, N\right)$. We call $\mathbf{s}$ a case when it is a case for some candidate $N$. If $\mathbf{s}$ is a case and $\bar{b}_{0} \in \zeta(0)\left(M_{\mathbf{x}}\right)$ realizes $q_{\mathbf{s}, 0}$, then we can choose $\overline{\mathbf{c}}_{\mathbf{s}, \bar{b}_{0}}=\left\langle\bar{c}_{\mathbf{x}, \bar{b}_{0}, t}: t \in I_{\mathbf{s}}\right\rangle$ such that $\operatorname{tp}\left(\bar{c}_{\mathbf{s}, \bar{b}_{0}, t}, \cup\left\{\bar{c}_{\mathbf{s}, \bar{b}_{0}, s}: s<_{I_{\mathbf{s}}} t\right\} \cup M_{\mathbf{x}}\right)$ is defined by the scheme $\Xi_{t}$ with the parameter $\bar{b}_{0}$, this type is determined by $\mathbf{s}, \bar{b}_{0}$ and $\mathbf{x}$ (though not the $\bar{c}_{\mathbf{s}, \bar{b}_{0}, t}$ 's themselves). Without loss of generality $t \in I_{\mathbf{x}} \Rightarrow \bar{c}_{\mathbf{s}, \bar{b}_{0}, t}=\bar{c}_{\mathbf{x}, t}$.

Now clearly

$(*)_{1}$ for a candidate $N$ we have $\mathbf{x}_{N} \in K_{\kappa, \theta}$ iff for every case $\mathbf{s}$ and $\bar{b}_{0} \in \zeta(0) N$ realizing $q_{\mathbf{s}, 0}$ there is no $\bar{b}_{1} \in{ }^{\ell g(\bar{y})} N$ such that $\mathbf{s}_{1}, \bar{b}_{0}, \bar{b}_{1}, \bar{b}_{2}=\bar{c}_{\mathbf{s}, \bar{b}_{0}}$ are as above.

So

$(*)_{2}$ for every case $\mathbf{s}$ and $\bar{b}_{0} \in{ }^{(0)}\left(M_{\mathbf{x}}\right)$ realizing $q_{\mathbf{s}, 0}$ and $\bar{b}_{1} \in{ }^{\ell g\left(\bar{z}_{\mathbf{s}, 1}\right)} M$, we cannot choose $\overline{\mathbf{c}}^{\prime}, \overline{\mathbf{c}}^{\prime \prime}$ realizing $\operatorname{tp}\left(\overline{\mathbf{c}}_{\mathbf{s}, \bar{b}_{0}}, M_{\mathbf{x}}\right)$ such that $\overline{\mathbf{c}}^{\prime}\left\lceil I_{\mathbf{x}}=\overline{\mathbf{c}}_{\mathbf{x}}=\overline{\mathbf{c}}^{\prime \prime}\left\lceil I_{\mathbf{x}}\right.\right.$ and $\mathfrak{C} \models \varphi_{\mathbf{s}}\left[\mathbf{d}_{\mathbf{x}}, \bar{c}_{t_{*}(\mathbf{s})}^{\prime}, \bar{b}_{1}, \overline{\mathbf{c}}^{\prime} \uparrow\left(I_{\mathbf{s}} \backslash\left\{t_{*}(\mathbf{s})\right\}\right)\right] \wedge \neg \varphi_{\mathbf{s}}\left[\mathbf{d}_{\mathbf{x}}, \bar{c}_{t_{*}}^{\prime \prime}(\mathbf{s}), \bar{b}_{1}, \overline{\mathbf{c}}^{\prime} \uparrow\left(I_{\mathbf{x}} \backslash\left\{t_{*}(\mathbf{s})\right\}\right)\right]$.

[Why? As then $\mathbf{x} \notin \mathrm{mxK}_{\kappa, \theta}$.]

Hence

$(*)_{3}$ for every case $\mathbf{s}$ and $\bar{b}_{0} \in \zeta(0)\left(M_{\mathbf{x}}\right)$ realizing $q_{\mathbf{s}, 0}$ and $\bar{b}_{1} \in{ }^{\ell g\left(\bar{z}_{\mathbf{s}, 1}\right)}\left(M_{\mathbf{x}}\right)$ there is a finite set $C=C_{\mathbf{s}}\left(\bar{b}_{0}, \bar{b}_{1}\right) \subseteq M_{\mathbf{x}}$ such that: if $N$ is a candidate which includes $\bar{b}_{0}, \bar{b}_{1}, C$ then there is no witness $\mathbf{w}$ against $N$ with $\mathbf{s}=\mathbf{s}_{\mathbf{w}}, \bar{b}_{0}=$ $\bar{b}_{\mathbf{w}, 0}, \bar{b}_{1}=\bar{b}_{\mathbf{w}, 1}$.

Also $(1.2(4))$ 
$(*)_{4}$ for $B \subseteq M$ of cardinality $<\kappa$ let $C(B)$ be a subset of $M$ of cardinality $\leq 2^{|B|+|T|}$ in which every $p \in \mathbf{S}^{<\omega}(B)$ is realized.

Lastly, let $F$ be defined for $B \in[M]^{<\kappa}, F(B)=\cup\left\{C_{\mathbf{s}}\left(\bar{b}_{0}, \bar{b}_{1}\right):\right.$ s a case and $\bar{b}_{0}, \bar{b}_{1}$ suitable sequences from $B\} \cup C(B)$.

Now the number of cases is $\leq \aleph_{0}+\aleph_{0}+\aleph_{0}+|T|+\theta+2^{|T|+|A|}=2^{|T|+|A|}$, so $F(B) \in[M] \leq \theta$. So we are done.

Theorem 2.12. The Existence Theorem If $\ell=0,1$ and $\operatorname{cf}(\kappa)>\theta \geq|T|$ and $\mathbf{x} \in K_{\lambda, \kappa, \theta}^{\ell}$ then there is $\mathbf{y}$ such that $\mathbf{x} \leq_{2} \mathbf{y} \in \operatorname{mxK}_{\lambda, \kappa, \theta}^{\ell}$.

Remark 2.13. 1) If we use $K_{\lambda, \kappa,<\theta}^{\ell}$ instead of " $\theta \geq|T|$ " we should demand "cf $(\theta)>$ $|T| "$.

2) We may get more. E.g. demand $I_{1}=I_{\mathbf{x}}, I_{2}$ is well ordered and $I_{\mathbf{y}}=I_{1} \cup I_{2}, I_{1}<$ $I_{2}$, i.e. $s_{1} \in I_{1} \wedge s_{2} \in I_{2} \Rightarrow s_{1}<_{I} s_{2}$.

$2 \mathrm{~A})$ Also this claim holds (by the same proof) when we replace clause $(f)$ in Definition 2.6 (1) by

$(f)_{2} \operatorname{tp}\left(\overline{\mathbf{c}}_{\mathbf{x}}, A_{\mathbf{x}}\right)$ is finitely satisfiable in $B_{\mathbf{x}}$.

Then in part (2) of the remark we may add

(*) for $t \in I_{2}, \operatorname{tp}\left(\bar{c}_{t}, M_{\mathbf{x}} \cup\left\{\bar{c}_{\mathbf{y}, s}: s<_{I_{\mathbf{y}}} t\right\}\right)$ is finitely satisfiable in $B_{\mathbf{y}}$.

See more in 2.33, 2.34 and 2.35 .

2B) In this case we may say "Clause (f) 1 , of 2.6 (1)" instead of Clause (f).

3) We can be more relaxed in the demands on $\left\langle\mathbf{x}_{\alpha}: \alpha<\theta^{+}\right\rangle$in the proof e.g. it suffices to demand

$\circledast)^{\prime}(a) \quad \mathbf{x}_{\alpha} \in K_{\lambda, \kappa, \theta}^{0}$,

(b) $\mathbf{x}_{\alpha}$ is $\leq_{1}$-increasing continuous, natural to demand " $\leq_{2}$-increasing", that is, $\overline{\mathbf{d}}_{\mathbf{x}_{\alpha}}=\overline{\mathbf{d}}_{\mathbf{x}_{0}}$ but not necessary,

(c) for each $\alpha<\theta^{+}$(or just for stationarily many $\alpha<\theta^{+}$) we have $\mathbf{x}_{\alpha}<_{1}^{+} \mathbf{x}_{\alpha+1}$.

[Why? Let $\overline{\mathbf{d}}_{\mathbf{x}_{1}}\left\lceil u_{\alpha}\left(u_{\alpha}\right.\right.$ finite) $t_{\alpha}, \varphi_{\alpha}\left(\bar{x}_{\alpha}, \bar{y}_{\alpha}, \bar{b}_{\alpha}\right)$ witness $\mathbf{x}_{\alpha}<_{1}^{+} \mathbf{x}_{\alpha+1}$ when $\alpha \in S_{0}$ where $S_{0}:=\left\{\alpha<\theta^{+}: \mathbf{x}_{\alpha}<_{1}^{+} \mathbf{x}_{\alpha+1}\right\}$. For $\alpha \in S_{0}$ let $h(\alpha)=\operatorname{Min}\left\{\gamma: \overline{\mathbf{d}}_{\mathbf{x}_{\alpha}}\left\lceil u_{\alpha}=\right.\right.$ $\overline{\mathbf{d}}_{\mathbf{x}_{\gamma}}\left\lceil u_{\alpha}\right.$, equivalently $u_{\alpha} \subseteq \operatorname{Dom}\left(\overline{\mathbf{d}}_{\mathbf{x}_{\gamma}}\right)$ and $\left.\operatorname{Rang}\left(\bar{b}_{\alpha}\right) \cap\left(A_{\mathbf{x}_{\alpha}}+\overline{\mathbf{c}}_{\mathbf{x}_{\alpha}}\right) \subseteq A_{\mathbf{x}_{\gamma}}+\overline{\mathbf{c}}_{\mathbf{x}_{\gamma}}\right\}$, clearly $h(\alpha)<\alpha$ for $\alpha$ limit $\in S_{0}$.

So by Fodor's Lemma for some $\beta<\alpha$ and $u$ the set $S=\left\{\delta \in S_{0}: \delta\right.$ is a limit ordinal as in clause (c) above $u_{\delta}=u$ and $h(\delta)=\beta$ \} is stationary. As $\theta \geq|T|$, for some 10 formula $\varphi$ the set $S_{2}:=\left\{\delta \in S_{1}: \varphi_{\delta}=\varphi\right\}$ is a stationary subset of $\theta^{+}$and we continue as in the proof.

4) How does part (3) of the remark help? E.g. if we like to get $\mathbf{y} \in \operatorname{mxK}_{\lambda, \kappa, \theta}^{\ell}$ which is normal and $\operatorname{Rang}\left(\overline{\mathbf{d}}_{\mathbf{y}}\right)$ is the universe of some $N \prec \mathfrak{C}$.

Proof. Assume this fails. We try to choose $\mathbf{x}_{\alpha}$ by induction on $\alpha<\theta^{+}$such that

$\circledast(a) \quad \mathbf{x}_{\alpha} \in K_{\lambda, \kappa, \theta}^{\ell}$,

\footnotetext{
$10_{\text {if }} \bar{c}_{t_{\alpha}, n}$ is infinite we let $u_{\alpha} \subseteq \ell g\left(\bar{c}_{t_{\alpha, 0}}^{\mathbf{x}_{\alpha}}\right)$ be finite such that $\mathfrak{C} \models \varphi_{\alpha}\left[\overline{\mathbf{d}}_{\mathbf{x}}, \bar{c}_{t_{\alpha, 0}}^{\mathbf{x}_{\alpha+1}}\left\lceil u_{\alpha}, \bar{b}_{\alpha}\right] \wedge\right.$

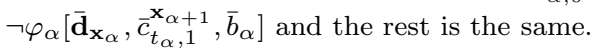


(b) $\mathbf{x}_{\beta} \leq_{2} \mathbf{x}_{\alpha}$ for $\beta<\alpha$,

(c) if $\alpha=\beta+1$ then $\mathbf{x}_{\beta}<_{2}^{+} \mathbf{x}_{\alpha}$, i.e. $\mathbf{x}_{\alpha}$ witness $\mathbf{x}_{\beta} \notin \operatorname{mxK} K_{\lambda, \kappa, \theta}^{\ell}$

(i.e. is like $\mathbf{y}$ in Definition 2.8);

(d) $\quad \mathbf{x}_{0}=\mathbf{x}$.

For $\alpha=0$ use clause (d), for $\alpha=\beta+1$ we use our assumption toward contradiction. For $\alpha$ limit use $2.7(3)$. Note that $\overline{\mathbf{d}}_{\mathbf{x}_{\alpha}}=\overline{\mathbf{d}}_{\mathbf{x}}$ for $\alpha<\theta^{+}$by clauses $(\mathrm{d})+(\mathrm{b})$.

Having carried the induction, for each $\alpha<\theta^{+}$there are $t_{\alpha}, \varphi_{\alpha}\left(\bar{x}, \bar{y}_{\alpha}, \bar{z}_{\alpha}\right), \bar{b}_{\alpha}$ satisfying:

$$
\begin{aligned}
&(*)_{\alpha}^{1} \text { (a) } t_{\alpha} \in I_{\mathbf{x}_{\alpha+1}} \backslash I_{\mathbf{x}_{\alpha}} \\
& \text { (b) } \varphi_{\alpha}\left(\bar{x}, \bar{y}_{\alpha}, \bar{z}_{\alpha}\right) \in \mathbb{L}\left(\tau_{T}\right) \\
&(c) \bar{b}_{\alpha} \subseteq M_{\mathbf{x}_{\alpha}} \cup \bigcup\left\{\bar{c}_{\mathbf{x}_{\alpha+1}, s}: s \in I_{\mathbf{x}_{\alpha+1}} \backslash\left\{t_{\alpha}\right\}\right\} \\
& \text { (d) } \lg \left(\bar{b}_{\alpha}\right)=\ell g\left(\bar{z}_{\alpha}\right), \ell g\left(\bar{y}_{\alpha}\right)=\ell g\left(\bar{c}_{\mathbf{x}_{\alpha+1}, t_{\alpha}, 0}\right) \\
& \text { (e) } \mathfrak{C} \models " \neg \varphi_{\alpha}\left[\overline{\mathbf{d}}_{\mathbf{x}}, \bar{c}_{\mathbf{x}_{\alpha+1}, t_{\alpha}, 0}, \bar{b}_{\alpha}\right] \wedge \varphi_{\alpha}\left[\overline{\mathbf{d}}_{\mathbf{x}}, \bar{c}_{\mathbf{x}_{\alpha+1}, t_{\alpha}, 1}, \bar{b}_{\alpha}\right] " \text { so } \\
& \lg (\bar{x})=\ell g\left(\overline{\mathbf{d}}_{\mathbf{x}}\right) \text { and } n_{t_{\alpha}} \geq 2 .
\end{aligned}
$$

Clearly the sequence $\left\langle t_{\alpha}: \alpha<\theta^{+}\right\rangle$is without repetitions. Now let $J_{\alpha} \subseteq I_{\mathbf{x}_{\alpha+1}} \backslash\left\{t_{\alpha}\right\}$ be finite such that $\bar{b}_{\alpha} \subseteq M_{\mathbf{x}_{\alpha}} \cup \bigcup\left\{\bar{c}_{x_{\alpha+1}, s}: s \in J_{\alpha}\right\}$. We can find a pair $\left(\varphi(\bar{x}, \bar{y}, \bar{z}), n_{*}, m_{*}\right)$ such that the set $S_{0}=\left\{\delta<\theta^{+}:\left|J_{\delta}\right| \leq n_{*}\right.$ and $\left.\varphi_{\delta}\left(\bar{x}, \bar{y}_{0}, \bar{z}_{0}\right)=\varphi(\bar{x}, \bar{y}, \bar{z})\right\}$ is infinite (even stationary). By Ramsey's theorem (or Fodor's Lemma) we can find an infinite (and even stationary) set $S_{1} \subseteq S_{0} \subseteq \theta^{+}$such that $\delta \in S_{1} \Rightarrow J_{\delta} \cap\left\{t_{\beta}: \beta \in S_{1}\right\}=\emptyset$. Note that there are $\leq \theta$ possibilities for $\varphi$, not necessarily $\leq|T|$ because though $\varphi_{\alpha}\left(\bar{x}, \bar{y}_{\alpha}, \bar{z}_{\alpha}\right)$ depend only on $\bar{x} \uparrow u$ for some finite $u \subseteq \ell g(\bar{x})$ there are $\leq \ell g\left(\overline{\mathbf{d}}_{\mathbf{x}}\right)+\aleph_{0}$ possibilities for $u$.

Next we shall prove that in this case $\varphi(\bar{x} ; \bar{y}, \bar{z})$ has the independence property (for $T$ ), a contradiction.

For every $w, v \subseteq S_{1}$ and $\eta \in{ }^{w} 2$ let, noting that $w=\operatorname{Dom}(\eta)$ :

$(*)_{2}(a) \quad A_{\eta, v}=A_{\mathbf{x}} \cup\left\{\bar{c}_{\mathbf{x}_{\beta+1}, t, n}: t \in J_{\beta}\right.$ and $n<n_{\mathbf{x}_{\beta+1}, t}$ for some $\left.\beta \in v\right\} \cup$ $\left\{\bar{c}_{\mathbf{x}_{\alpha+1}, t_{\alpha}, \eta(\alpha)}: \alpha \in w\right\}$

(b) $f_{\eta, v}$ is the function with:

( $\alpha$ ) domain $A_{\eta, v}$,

( $\beta) \quad$ is the identity on $A_{\mathbf{x}} \cup\left\{\bar{c}_{\mathbf{x}_{\beta+1}, t, n}: t \in J_{\beta}\right.$ and $n<n_{\mathbf{x}_{\beta+1}, t}$ for some $\left.\beta \in v\right\}$,

$(\gamma) f_{\eta, v}\left(\bar{c}_{\mathbf{x}_{\alpha+1}, t_{\alpha}, \eta(\alpha)}\right)=\bar{c}_{\mathbf{x}_{\alpha+1}, t_{\alpha}, 0}$ for $\alpha \in w=\operatorname{Dom}(\eta)$.

Now

$(*)_{3} f_{\eta, v}$ is an elementary mapping.

[Why? Without loss of generality $w \subseteq v$ are finite so $\subseteq \alpha(*)$ for some $\alpha(*)<\theta^{+}$ and prove this by induction on $|v|$. We just use: for $\alpha \in v$ the sequence $\left\langle\bar{c}_{\mathbf{x}_{d(*)}, t, n}\right.$ : $\left.n<n_{\mathbf{x}_{\alpha(*)}, t}\right\rangle$ is indiscernible over $A_{\mathbf{x}_{\alpha(*)}} \cup\left\{\bar{c}_{\mathbf{x}_{\alpha(*)}, s}: s \in I_{\mathbf{x}_{\alpha(*)}} \backslash\{t\}\right\}$, by Definition 2.6(1), Clause (e).]

Now let $g_{\eta, v} \in \operatorname{aut}(\mathfrak{C})$ extends $f_{\eta, v}$. So $\alpha \in v \Rightarrow g_{\eta, v}\left(\bar{b}_{\alpha}\right)=f_{\eta}\left(\bar{b}_{\alpha}\right)=\bar{b}_{\alpha}$ and $g_{\eta, \nu}\left(\bar{c}_{\mathbf{x}_{\alpha+1}, t_{\alpha}, \eta(\alpha)}\right)=\bar{c}_{\mathbf{x}_{\alpha+1}, t_{\alpha}, 0}$ for $\alpha \in w$; hence by the choice of $J_{\alpha}$ so for $\eta \in w_{2}$ we have

$$
(*)_{4} \mathfrak{C} \models \varphi\left[g_{\eta, v}\left(\overline{\mathbf{d}}_{\mathbf{x}}\right), \bar{c}_{\mathbf{x}_{\alpha+1}, t_{\alpha}, 0}, \bar{b}_{\alpha}\right] \text { iff } \eta(\alpha)=1 .
$$


So $\left\langle\varphi\left(\bar{x}, \bar{c}_{\mathbf{x}_{\alpha+1}, t_{\alpha}, 0}, \bar{b}_{\alpha}\right): t \in u\right\rangle$ is an independent sequence of formulas, see 0.4(a); as $w$ is any subset of $S_{1}$ we get a contradiction as promised.

Claim 2.14. The Weak Orthogonality Claim

Assume $\ell=0,1$ and $\mathbf{x} \in \operatorname{mxK}_{\lambda, \kappa, \theta}^{\ell}$.

1) If $m<\omega, B^{\prime} \subseteq M_{\mathbf{x}}$ and $\left|B^{\prime}\right|<\kappa$ and $q \in \mathbf{S}^{m}\left(M_{\mathbf{x}} \cup C_{\mathbf{x}}\right)$ does not split over $B^{\prime}$ then $\operatorname{tp}\left(\overline{\mathbf{d}}_{\mathbf{x}}, M_{\mathbf{x}} \cup C_{\mathbf{x}}\right)$ is weakly orthogonal to $q$.

1A) It suffice.12 that $q=\operatorname{tp}\left(\bar{c}_{1}{ }^{\wedge} \bar{c}_{2}, M_{\mathbf{x}} \cup C_{\mathbf{x}}\right)$ and $\operatorname{tp}\left(\bar{c}_{1}, M_{\mathbf{x}}\right)$ does not split over some $B^{\prime} \in[M]^{<\kappa}, \operatorname{tp}\left(C_{\mathbf{x}}, M_{\mathbf{x}}+\bar{c}_{1}\right)$ does not split over $B_{\mathbf{x}}$ and $\operatorname{tp}\left(\bar{c}_{2}, M_{\mathbf{x}}+\bar{c}_{1}+C_{\mathbf{x}}\right)$ does not split over some $B^{\prime \prime \prime} \in\left[M_{\mathbf{x}}\right]^{<\kappa}$.

2) If $\mathbf{x}_{\alpha} \in \operatorname{mxK}_{\lambda, \kappa, \theta}^{\ell}$ for $\alpha<\delta$ is $\leq_{1}$-increasing, $\delta<\theta^{+}$and $\mathbf{x}_{\delta}:=\cup\left\{\mathbf{x}_{\alpha}: \alpha<\delta\right\}$ belongs to $K_{\lambda, \kappa, \theta}^{\ell}($ recall $2.7(3))$ then it also belongs to $\operatorname{mxK}_{\lambda, \kappa, \theta}^{\ell}$.

Proof. 1) Assume toward contradiction that those types are not weakly orthogonal. Let $q=q(\bar{y}), \bar{y}=\left\langle y_{k}: k<m\right\rangle$ and let $\bar{x}=\left\langle x_{\alpha}: \alpha<\alpha_{\mathbf{x}}\right\rangle$ recalling $\alpha_{\mathbf{x}}=\ell g\left(\overline{\mathbf{d}}_{\mathbf{x}}\right)$ and $p(\bar{x})=\operatorname{tp}\left(\overline{\mathbf{d}}_{\mathbf{x}}, M_{\mathbf{x}} \cup C_{\mathbf{x}}\right)$. So for some formula $\varphi(\bar{x}, \bar{y}, \bar{z})$ and $\bar{e} \in{ }^{\ell(\bar{z})}\left(M_{\mathbf{x}} \cup C_{\mathbf{x}}\right)$ the type $p(\bar{x}) \cup q(\bar{y})$ does not decide $\varphi(\bar{x}, \bar{y}, \bar{e})$, i.e. $r_{\mathbf{t}}(\bar{x}, \bar{y})=p(\bar{x}) \cup q(\bar{y}) \cup\left\{\varphi(\bar{x}, \bar{y}, \bar{e})^{\mathbf{t}}\right\}$ is consistent (= finitely satisfiable in $\mathfrak{C}$ ) for $\mathbf{t}=0,1$ and let $\bar{c}_{0}^{\prime}, \bar{c}_{1}^{\prime}$ be such that $\overline{\mathbf{d}}^{\wedge} \bar{c}_{\mathbf{t}}^{\prime}$ realizes $r_{\mathbf{t}}(\bar{x}, \bar{y})$ for $\mathbf{t}=0,1$. Now it cannot be that $\operatorname{tp}\left(\bar{c}_{\mathbf{t}}^{\prime}, M_{\mathbf{x}} \cup C_{\mathbf{x}} \cup \overline{\mathbf{d}}_{\mathbf{x}}\right)$ does not split over $B^{\prime}$ for both $\mathbf{t}=0,1$ (by $1.2(1)$, as every $p \in \mathbf{S}^{<\omega}\left(B^{\prime}\right)$ is realized in $M$ recalling $M$ is $\kappa$-saturated and $\left.\kappa>\left|B^{\prime}\right|\right)$. So choose $\bar{c}_{0} \in\left\{\bar{c}_{0}^{\prime}, \bar{c}_{1}^{\prime}\right\}$ such that the type $\operatorname{tp}\left(\bar{c}_{0}, M_{\mathbf{x}} \cup C_{\mathbf{x}} \cup \overline{\mathbf{d}}\right)$ splits over $B^{\prime}$.

Now by $1.2(1)$ there is $\bar{c}_{1} \in{ }^{m} \mathfrak{C}$ such that $\operatorname{tp}\left(\bar{c}_{1}, M_{\mathbf{x}} \cup C_{\mathbf{x}} \cup \overline{\mathbf{d}}_{\mathbf{x}} \cup \bar{c}_{0}\right)$ extends $q(\bar{y})$ and does not split over $B^{\prime}$. Hence also $\operatorname{tp}\left(\bar{c}_{1}, M_{\mathbf{x}} \cup C_{\mathbf{x}} \cup \overline{\mathbf{d}}_{\mathbf{x}}\right)$ does not split over $B^{\prime}$, hence it is different from $\operatorname{tp}\left(\bar{c}_{0}, M_{\mathbf{x}} \cup C_{\mathbf{x}} \cup \overline{\mathbf{d}}_{\mathbf{x}}\right)$. We can continue and choose $\bar{c}_{n}(n=2,3 \ldots)$ realizing the complete type over $M_{\mathbf{x}} \cup C_{\mathbf{x}} \cup \overline{\mathbf{d}}_{x} \cup \bigcup\left\{\bar{c}_{k}: k<n\right\}$ which extends $q$ and does not split over $B^{\prime}$. Hence

$(*)_{0}$ for every $n<\omega, \operatorname{tp}\left(\bar{c}_{n}, M_{\mathbf{x}} \cup C_{\mathbf{x}} \cup \bigcup\left\{\bar{c}_{k}: k<n\right\}\right)$ extend $q(\bar{y})$ and does not split over $B^{\prime}$.

So by $1.2(2)$

$(*)_{1}\left\langle\bar{c}_{n}: n<\omega\right\rangle$ is an indiscernible sequence over $M_{\mathbf{x}} \cup C_{\mathbf{x}}$.

Also (by induction on $\gamma \leq \omega$ ) by $1.2(3)$ we have:

$(*)_{2}$ if $t \in I_{\mathbf{x}}$ then $\left\langle\bar{c}_{\mathbf{x}, t, n}: n<n_{t}\right\rangle$ is an indiscernible sequence over $M_{\mathbf{x}} \cup\left\{\bar{c}_{\mathbf{x}, s, m}\right.$ : $s \in I \backslash\{t\}\} \cup\left\{\bar{c}_{n}: n<\gamma\right\}$.

Now we define $\mathbf{y}=\left(M_{\mathbf{y}}, B_{\mathbf{y}}, \overline{\mathbf{c}}_{\mathbf{y}}, \overline{\mathbf{d}}_{\mathbf{y}}\right)$ by

$\circledast(a) \quad M_{\mathbf{y}}=M_{\mathbf{x}}$,

(b) $B_{\mathbf{y}}=B_{\mathbf{x}} \cup B^{\prime}$,

(c) $\overline{\mathbf{d}}_{\mathbf{y}}=\overline{\mathbf{d}}_{\mathbf{x}}$,

(d) $I_{\mathbf{y}}=I_{\mathbf{x}} \cup\{s(*)\}$ were $\left[t \in I_{\mathbf{x}} \Rightarrow t<_{I_{\mathbf{y}}} s(*)\right]$,

(e) $\overline{\mathbf{c}}_{\mathbf{y}} \uparrow I_{\mathbf{x}}=\overline{\mathbf{c}}_{\mathbf{x}}$,

(f) $\quad n_{\mathbf{y}, s(*)}=\omega($ or any number $\in[2, \omega])$ and $\bar{c}_{\mathbf{y}, s(*), n}=\bar{c}_{n}$.

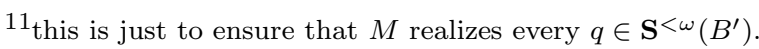

12 even more as we can increase the linear order $I_{\mathbf{x}}$.
} 
Now $\mathbf{y}$ contradicts the assumption $\mathbf{x} \in \operatorname{mxK}_{\lambda, \kappa, \theta}^{\ell}$.

1A) Similarly (recalling that we can use $\left\{s_{1}\right\}+I_{\mathbf{x}}$ ).

2) Easy.

The following claim is a crucial step toward proving the Type Decomposition Theorem 2.4.

Claim 2.15. If $\mathbf{x} \in \operatorname{mxK}_{\lambda, \kappa, \theta}^{\ell}$ (or just $\mathbf{x} \in K$ and $\circledast$ below) then for every $A \subseteq M_{\mathbf{x}}$ of cardinality $<\kappa$ and $\varphi=\varphi(\bar{x}, \bar{y}, \bar{z}) \in \mathbb{L}\left(\tau_{T}\right)$ satisfying $\ell g(\bar{x})=\ell g\left(\overline{\mathbf{d}}_{\mathbf{x}}\right), \ell g(\bar{z})=$ $\ell g\left(\bar{c}_{\mathbf{x}}\right), \ell g(\bar{y})=m$, there is $\psi\left(\bar{x}, \bar{e}, \overline{\mathbf{c}}_{\mathbf{x}}\right) \in \operatorname{tp}\left(\overline{\mathbf{d}}_{\mathbf{x}}, M_{\mathbf{x}} \cup C_{\mathbf{x}}\right)$ satisfying $\bar{e} \in{ }^{\omega>} M_{\mathbf{x}}$ such that $\psi\left(\bar{x}, \bar{e}, \bar{c}_{\mathbf{x}}\right) \vdash\left\{\varphi\left(\bar{x}, \bar{b}, \overline{\mathbf{c}}_{\mathbf{x}}\right)^{\mathbf{t}}: \bar{b} \in{ }^{\ell g(\bar{y})} A\right.$ and $\mathbf{t} \in\{0,1\}$ are such that $\mathfrak{C} \models$ $\left.\varphi\left[\overline{\mathbf{d}}_{\mathbf{x}}, \bar{b}, \overline{\mathbf{c}}_{\mathbf{x}}\right]^{\mathbf{t}}\right\}$ where

$\circledast$ if $q(\bar{y}) \in \mathbf{S}^{\ell g(\bar{y})}\left(M_{\mathbf{x}} \cup \overline{\mathbf{c}}_{\mathbf{x}}\right)$ is finitely satisfiable in some $A \subseteq M_{\mathbf{x}}$ of cardinality $<\kappa$ then $q(\bar{y})$ is weakly orthogonal to $\operatorname{tp}\left(\overline{\mathbf{d}}_{\mathbf{x}}, M_{\mathbf{x}} \cup C_{\mathbf{x}}\right)$.

Proof. By 2.14 if $\mathrm{x} \in \mathrm{mxK}_{\lambda, \kappa, \theta}$ then $\circledast$ holds; hence we can in any case assume $\circledast$.

Let $p(\bar{x})=\operatorname{tp}\left(\overline{\mathbf{d}}_{\mathbf{x}}, M \cup C_{\mathbf{x}}\right)$, so $\bar{x}=\left\langle x_{i}: i<\ell g\left(\overline{\mathbf{d}}_{\mathbf{x}}\right)\right\rangle$ and recalling $\bar{y}=\left\langle y_{\ell}: \ell<\right.$ $m\rangle$ we define a set $r=r(\bar{y})$ as follows:

$$
r(\bar{y}):=\left\{(\exists \bar{x})\left(\varphi\left(\bar{x}, \bar{y}, \bar{c}_{\mathbf{x}}\right)^{\mathbf{t}} \wedge \psi\left(\bar{x}, \bar{a}, \overline{\mathbf{c}}_{\mathbf{x}}\right)\right): \begin{array}{ll}
\mathbf{t} \in\{0,1\} \text { and } \\
& \left.\psi\left(\bar{x}, \bar{a}, \overline{\mathbf{c}}_{\mathbf{x}}\right) \in p(\bar{x}) \text { and } \bar{a} \in{ }^{\omega>}\left(M_{\mathbf{x}}\right)\right\} .
\end{array}\right.
$$

Now

$\odot_{1} r(\bar{y})$ is not finitely satisfiable in ${ }^{m} A$.

[Why? If $r(\bar{y})$ is finitely satisfiable in ${ }^{m} A$, then there is an ultrafilter $\mathscr{D}$ on ${ }^{m} A$ such that for every $\vartheta\left(\bar{y}, \bar{a}, \bar{c}_{\mathbf{x}}\right) \in r(\bar{y})$, the set $\left\{\bar{b}: \bar{b} \in{ }^{m} A\right.$ and $\left.\models \vartheta\left[\bar{b}, \bar{a}, \bar{c}_{\mathbf{x}}\right]\right\}$ belongs to $\mathscr{D}$. Let $q(\bar{y})=\operatorname{Av}\left(\mathscr{D}, M_{\mathbf{x}} \cup \bar{c}_{\mathbf{x}}\right)$, clearly $q(\bar{y}) \in \mathbf{S}^{m}\left(M_{\mathbf{x}} \cup \overline{\mathbf{c}}_{\mathbf{x}}\right)$ is finitely satisfiable in ${ }^{m} A, A \subseteq M$ and $|A|<\kappa$. Let $\bar{b}^{*} \in{ }^{m} \mathfrak{C}$ realize $q(\bar{y})$, so $\left(\psi\left(\bar{x}, \bar{a}, \overline{\mathbf{c}}_{\mathbf{x}}\right) \in\right.$ $p(\bar{x})) \wedge \mathbf{t} \in\{0,1\} \Rightarrow \mathfrak{C} \models(\exists \bar{x})\left(\varphi\left(\bar{x}, \bar{b}^{*}, \overline{\mathbf{c}}_{\mathbf{x}}\right)^{\mathbf{t}} \wedge \psi\left(\bar{x}, \bar{a}, \overline{\mathbf{c}}_{\mathbf{x}}\right)\right)$. Why? This holds by the choices of $r(\bar{y}), \mathscr{D}$ and $\bar{b}^{*}$. As $p(\bar{x})$ is closed under conjunctions it follows that $p(\bar{x}) \cup\left\{\varphi\left(\bar{x}, \bar{b}^{*}, \overline{\mathbf{c}}_{\mathbf{x}}\right)^{\mathbf{t}}\right\}$ is finitely satisfiable in $\mathfrak{C}$ for $\mathbf{t}=0,1$. But this contradicts the assumption $\circledast$.]

Hence for some $n<\omega$ and $\psi_{\ell}\left(\bar{x}, \bar{a}_{\ell}, \overline{\mathbf{c}}_{\mathbf{x}}\right) \in p(\bar{x})$ for $\ell<n$ we have

$\odot_{2}$ for no $\bar{b} \in{ }^{m} A$ do we have $(\exists \bar{x})\left(\varphi\left(\bar{x}, \bar{b}, \overline{\mathbf{c}}_{\mathbf{x}}\right)^{\mathbf{t}} \wedge \psi_{\ell}\left(\bar{x}, \bar{a}_{\ell}, \bar{c}_{\mathbf{x}}\right)\right)$ for $\ell<n, \mathbf{t} \in$ $\{0,1\}$.

Let $\psi\left(\bar{x}, \bar{a}, \overline{\mathbf{c}}_{\mathbf{x}}\right)=\bigwedge_{\ell<n} \psi_{\ell}\left(\bar{x}, \bar{a}_{\ell}, \overline{\mathbf{c}}_{\mathbf{x}}\right)$, so clearly

$\odot_{3}(a) \quad \bar{a} \in \omega>\left(M_{\mathbf{x}}\right)$,

(b) $\psi\left(\bar{x}, \bar{a}, \overline{\mathbf{c}}_{\mathbf{x}}\right) \in p(\bar{x})$,

(c) for no $\bar{b} \in{ }^{m} A$ do we have $\bigwedge_{\mathbf{t}=0}^{1}(\exists \bar{x})\left(\varphi\left(\bar{x}, \bar{b}, \overline{\mathbf{c}}_{\mathbf{x}}\right)^{\mathbf{t}} \wedge \psi\left(\bar{x}, \bar{a}, \overline{\mathbf{c}}_{\mathbf{x}}\right)\right)$.

So for every $\bar{b} \in{ }^{m} A$ for some $\mathbf{t}=\mathbf{t}(\bar{b}) \in\{0,1\}$ we have $\mathfrak{C} \models \neg(\exists \bar{x})\left(\varphi\left(\bar{x}, \bar{b}, \overline{\mathbf{c}}_{\mathbf{x}}\right)^{\mathbf{t}} \wedge\right.$ $\left.\psi\left(\bar{x}, \bar{a}, \overline{\mathbf{c}}_{\mathbf{x}}\right)\right)$ hence $\psi\left(\bar{x}, \bar{a}, \overline{\mathbf{c}}_{\mathbf{x}}\right) \vdash \neg \varphi\left(\bar{x}, \bar{b}, \overline{\mathbf{c}}_{\mathbf{x}}\right)^{\mathbf{t}(\bar{b})}$. As $\psi\left(\bar{x}, \bar{a}, \overline{\mathbf{c}}_{\mathbf{x}}\right) \in p(\bar{x})=\operatorname{tp}\left(\overline{\mathbf{d}}_{\mathbf{x}}, M_{\mathbf{x}} \cup\right.$ $\left.\mathbf{c}_{\mathbf{x}}\right)$ it follows that $\neg \varphi\left(\bar{x}, \bar{b}, \overline{\mathbf{c}}_{\mathbf{x}}\right)^{\mathbf{t}(\bar{b})} \in p(\bar{x})$.

So $\psi\left(\bar{x}, \bar{a}, \overline{\mathbf{c}}_{\mathbf{x}}\right)$ is as required. 
Claim 2.16. Assume $\mathbf{x} \in \operatorname{mxK}_{\lambda, \kappa, \theta}^{\ell}$. If $B^{\prime} \subseteq M_{\mathbf{x}},\left|B^{\prime}\right|<\kappa, \bar{d} \in{ }^{m}\left(D_{\mathbf{x}}\right), \bar{c} \in$ $\omega>\left(C_{\mathbf{x}}\right), \varphi=\varphi(\bar{x}, \bar{y}, \bar{z}), \ell g(\bar{x})=\ell g(\bar{d}), \ell g(\bar{z})=\ell g(\bar{c})$ then for some $\psi\left(\bar{x}, \bar{y}^{\prime}, \bar{z}^{\prime}\right)$ and $\bar{e} \in^{\ell g\left(\bar{y}^{\prime}\right)}\left(M_{\mathbf{x}}\right)$ we have $\models \psi[\bar{d}, \bar{e}, \bar{c}]$ and

$$
\psi(\bar{x}, \bar{e}, \bar{c}) \vdash\left\{\varphi(\bar{x}, \bar{b}, \bar{c})^{\mathbf{t}}: \bar{b} \in^{\ell g(\bar{y})}\left(B^{\prime}\right) \text { and } \mathfrak{C} \models \varphi[\bar{d}, \bar{b}, \bar{c}]^{\mathbf{t}} \text { and } \mathbf{t} \in\{0,1\}\right\} .
$$

Proof. This just reformulates 2.15, see more in 2.20(0).

Now at last

Proof. Proof of 2.4, The Type Decomposition Theorem

By 2.7(4) there is $\mathbf{x} \in \mathrm{K}_{\kappa, \leq \theta}^{1}$ such that $\mathbf{d}_{\mathbf{x}}=\overline{\mathbf{d}}$ and $M_{\mathbf{x}}=M$. By the Existence Theorem 2.12 without loss of generality $\mathbf{x} \in \operatorname{mxK}_{\kappa, \leq \theta}^{1}$. Clearly $\left(\mathbf{P}_{\mathbf{x}, \theta}, \leq_{\mathbf{x}, \theta}\right)$ is a partial order. Assume that $\alpha(*)<\kappa$ and $p_{\alpha} \in \mathbf{P}_{\mathbf{x}, \theta}$ for $\alpha<\alpha(*)$. Let $B=\bigcup\left\{\operatorname{Dom}\left(p_{\alpha}\right): \alpha<\alpha(*)\right\} \cup B_{\mathbf{x}}$, so $B \subseteq M_{\mathbf{x}}$ has cardinality $<\kappa$. Hence by 2.16 for every $v \subseteq \ell g\left(\overline{\mathbf{c}}_{\mathbf{x}}\right)$ and $\varphi=\varphi(\bar{x}, \bar{y}, \bar{z})$ satisfying $\ell g(\bar{z})=\ell g\left(\overline{\mathbf{c}}_{\mathbf{x}}\right), \ell g(\bar{x})=$ $\ell g\left(\overline{\mathbf{d}}_{\mathbf{x}}\right), \ell g(\bar{y})<\omega$ there is $\psi=\psi_{\varphi}\left(\bar{x}, \bar{e}_{\varphi}, \overline{\mathbf{c}}_{\mathbf{x}}\right) \in \operatorname{tp}\left(\overline{\mathbf{d}}_{\mathbf{x}}, M \cup \overline{\mathbf{c}}_{\mathbf{x}}\right)$ where $\bar{e}_{\varphi} \in{ }^{\ell g(\bar{y})}\left(M_{\mathbf{x}}\right)$ such that $\psi_{\varphi}\left(\bar{x}, \bar{e}_{\varphi}, \overline{\mathbf{c}}_{\mathbf{x}}\right) \vdash\left\{\varphi\left(\bar{x}, \bar{b}, \overline{\mathbf{c}}_{\mathbf{x}}\right)^{\mathbf{t}}: \bar{b} \in{ }^{\ell g(\bar{y})} B\right.$ and $\mathfrak{C} \models \varphi\left[\overline{\mathbf{d}}_{\mathbf{x}}, \bar{b}, \overline{\mathbf{c}}_{\mathbf{x}}\right]^{\mathbf{t}}$ and $\mathbf{t} \in\{0,1\}\}$.

Let $A=\cup\left\{\operatorname{Rang}\left(\bar{e}_{\varphi}\right): \varphi\right.$ as above $\}$, clearly $|A| \leq \theta$ and let $p=\operatorname{tp}\left(\overline{\mathbf{d}}_{\mathbf{x}}, A \cup \overline{\mathbf{c}}_{\mathbf{x}}\right)$; it is an upper bound, as required.

Discussion 2.17. The type decomposition theorems say that we can analyze a type $p \in \mathbf{S}(M)$ in two steps; first $\operatorname{tp}(\bar{c}, M)$ does not split over some "small" $B \subseteq M$. Second, $\operatorname{tp}(\bar{d}, \bar{c}+M)$ is like a type in the theory of trees and lastly without loss of generality by 2.7(6) some initial segment of $\bar{d}$ realizes $p$. As an example, see Sh:877:

Exercise 2.18. Let $T=T_{\text {ord }}$ be $\operatorname{Th}(\mathbb{Q},<)$ and if $M \prec \mathfrak{C}_{T}, M$ is $\kappa$-saturated, in the main case and $p \in \mathbf{S}(M) \underline{\text { then }}$

(a) $p$ induces a cut $\bar{C}_{p}$ of $M$ where $\bar{C}_{p}=\left\langle C_{p, 1}, C_{p, 2}\right\rangle, C_{p, 1}=\{a \in M:(a<$ $x) \in p\}$ and $C_{p, 2}=M \backslash C_{p, 1}$

(b) now $\bar{C}_{p}$ has a pair $\left(\kappa_{1}, \kappa_{2}\right)$ of cofinalities, that is $\kappa_{1}=\operatorname{cf}\left(C_{p, 1},<_{M}\right), \kappa_{2}=$ $\operatorname{cf}\left(C_{p, 2},>_{M}\right)$ and $\max \left\{\kappa_{1}, \kappa_{2}\right\} \geq \kappa$,

(c) now $p$ does not split over some subset $B$ of $M$ of cardinality $\leq \lambda<\kappa \underline{\text { iff }}$ $\min \left\{\kappa_{1}, \kappa_{2}\right\} \leq \lambda$

(d) for every $B \subseteq M$ of cardinality $<\min \left\{\kappa_{1}, \kappa_{2}\right\}$ for some $\varphi(x, \bar{a}) \in p$ we have $\varphi(x, \bar{a}) \vdash p\left\lceil B\right.$ (i.e. $p$ under $\vdash$ is $\min \left\{\kappa_{1}, \kappa_{2}\right\}$-directed); in fact we can add that for some $a_{1} \in C_{p, 1}$ and $a_{2} \in C_{p, 2}$ we have $\varphi(x, \bar{a})=a_{1}<x<a_{2}$,

(e) so for $\kappa=\min \left\{\kappa_{1}, \kappa_{2}\right\}$ we have a decomposition which is trivial in some sense: either we have $\bar{c}=<>$ or we have $\bar{d}=\bar{c}$,

$(f)$ if e.g. $\kappa_{1}<\kappa_{2}$ and $B_{1}$ is an unbounded subset of $C_{p, 1}$ of cardinality $\kappa_{1}$ and $c, d$ realize in $\mathfrak{C}$ (where $M \prec \mathfrak{C}$ ) the type $p$ and $\mathfrak{C} \models c<d$, then $\operatorname{tp}(c, M)$ is finitely satisfiable in $B_{1}$ and for every $A \subseteq M$ of cardinality $<\kappa_{2}$ for some formula $\varphi(x, \bar{a}) \in p$ we have $\varphi(x, \bar{a}) \wedge(c<x) \vdash \operatorname{tp}(d, A \cup\{c\})$.

Discussion 2.19. Note: if $T$ is stable and $\mathbf{x} \in \operatorname{mxK}_{\kappa, \theta}$ is normal then $\overline{\mathbf{d}}_{\mathbf{x}} \subseteq$ $\operatorname{dcl}\left(\operatorname{Rang}\left(\overline{\mathbf{c}}_{\mathbf{x}}\right)\right)$ recalling $\operatorname{dcl}(A)=\{b: b$ is definable over $A$, equivalently $\operatorname{tp}(c, A)=$ $\operatorname{tp}(b, A) \Rightarrow c=b\}$. 
Claim 2.20. 0) In 2.16 if $\operatorname{cf}(\kappa)>\theta+|T| \underline{\text { then }}$ we can choose $\psi=\psi\left(\bar{x}, \bar{y}^{\prime}, \bar{z}^{\prime}\right)$ such that it depends on $\mathbf{x}, \bar{d}, \varphi(\bar{x}, \bar{y}, \bar{z})$ but not on $B^{\prime}$.

OA) In [2.16 if $\operatorname{cf}(\kappa)>2^{\theta+|T|} \underline{\text { then }}$ we can fix also $q=\operatorname{tp}\left(\bar{e}, C_{\mathbf{x}} \cup \overline{\mathbf{d}}_{\mathbf{x}}\right)$. If $\operatorname{cf}(\kappa)>$ $2^{\theta+|T|+\left|B_{\mathbf{x}}\right|}$ then moreover we can fix $q=\operatorname{tp}\left(\bar{e}, B_{\mathbf{x}} \cup \mathbf{c}_{\mathbf{x}} \cup \overline{\mathbf{d}}_{\mathbf{x}}\right)$.

1) Assume that $\mathbf{x} \in K_{0}$ and $\varphi=\varphi(\bar{x}, \bar{y}, \bar{z}) \in \mathbb{L}\left(\tau_{T}\right)$ are such that $\bar{y}, \bar{z}$ are finite and $\ell g(\bar{x})=\ell g\left(\overline{\mathbf{d}}_{\mathbf{x}}\right)$.

$\underline{\text { Then }}$ the following set is finite: $J=J_{\mathbf{x}, \varphi}=\left\{t \in I_{\mathbf{x}}:\right.$ there are $\bar{\alpha}=\left\langle\alpha_{\ell}: \ell<\right.$ $\ell g(\bar{y})\rangle$ and a sequence $\bar{b} \in{ }^{\ell g(\bar{y})}\left(A_{\mathbf{x}} \cup\left\{\bar{c}_{\mathbf{x}, s}: s \in I_{\mathbf{x}} \backslash\{t\}\right\}\right)$ such that $\alpha_{\ell} \leq \ell g\left(\bar{c}_{\mathbf{x}, t, 0}\right)$ and $\left.\left.\mathfrak{C} \models \varphi\left[\overline{\mathbf{d}}_{\mathbf{x}},\left\langle\left(\bar{c}_{\mathbf{x}, t, 0}\right)_{\alpha_{\ell}}: \ell<\ell g(\bar{y})\right\rangle, \bar{b}\right] \wedge \neg \varphi\left[\bar{d}_{\mathbf{x}},\left\langle\left(\bar{c}_{\mathbf{x}, t, 1}\right)_{\alpha_{\ell}}: \ell<\ell g(\bar{y})\right\rangle, \bar{b}\right)\right]\right\}$.

2) Moreover, the bound depends just on $\varphi$ and $T$.

3) For any $\mathbf{x} \in K_{<\aleph_{0},<\aleph_{0}}$ and $\varphi$ there is $\mathbf{y}$ satisfying $\mathbf{x} \leq_{2} \mathbf{y} \in K_{<\aleph_{0},<\aleph_{0}}$ such that $I_{\mathbf{y}} \backslash I_{\mathbf{x}}$ is finite and the local version of maximality holds, i.e.

$$
\mathbf{y} \leq_{2} \mathbf{z} \in K_{<\aleph_{0},<\aleph_{0}} \Rightarrow J_{\mathbf{z}, \varphi}=J_{\mathbf{y}, \varphi} .
$$

4) For any $\mathbf{x} \in K_{<\aleph_{0},<\aleph_{0}}$ and sequence $\left\langle\varphi_{n}: n<\omega\right\rangle$ we can find $\left\langle\mathbf{x}_{n}: n<\omega\right\rangle$ such that $\mathbf{x}_{0}=\mathbf{x}, \mathbf{x}_{n} \leq_{2} \mathbf{x}_{n+1} \in K_{<\aleph_{0},<\aleph_{0}}, I_{\mathbf{x}_{n}} \backslash I_{\mathbf{x}}$ finite and $\mathbf{x}_{n+1}, \varphi_{n}$ satisfies the demands on $\mathbf{y}, \varphi$ above for every $n$.

Proof. 0) As there are $\leq \theta+|T|<\operatorname{cf}(\kappa)$ possible choices of $\psi$ and the set of possible $B$ 's is $(\operatorname{cf}(\kappa))$-directed.

$0 A)$ Similarly.

1),2) Let $n_{1}$ be minimal such that: for no $\bar{b}_{\ell} \in{ }^{\ell g(\bar{y})} \mathfrak{C}, \bar{c}_{\ell} \in{ }^{\ell g(\bar{z})} \mathfrak{C}$ for $\ell<n_{1}$ is $\left\langle\varphi\left(\bar{x}, \bar{b}_{\ell}, \bar{c}_{\ell}\right): \ell<n_{1}\right\rangle$ an independent sequence of formulas, exist as $T$ is a dependent theory. Let $n_{2}$ be minimal such that if $u_{i} \in\left[n_{2} \backslash\{i\}\right]^{\ell g(\bar{z})}$ for $i<n_{2}$ then for some $v \in\left[n_{2}\right]^{n_{1}}$ we have $i, j \in v \Rightarrow i \notin u_{j}$ (the $\Delta$-system lemma for finite sets, see 0.5).

Now $n_{2}$ is a bound as required by the proof of 2.12 .

3),4) Follows.

Conclusion 2.21. If $\mathbf{x} \in \operatorname{mxK}_{\lambda, \kappa, \theta}^{\ell}$ and $\operatorname{cf}(\kappa)>\theta+|T| \underline{\text { then }}$ we can find $\bar{e} \in$ ${ }^{\theta} \mathfrak{C}$ and $\left\langle\psi_{\varphi(\bar{x}, \bar{y}, \bar{z})}\left(\bar{x}, \bar{e}, \overline{\mathbf{c}}_{\mathbf{x}}\right): \varphi(\bar{x}, \bar{y}, \bar{z}) \in \mathbb{L}\left(\tau_{T}\right)\right\rangle$ satisfying $\ell g(\bar{x})=\ell g\left(\overline{\mathbf{d}}_{\mathbf{x}}\right), \ell g(\bar{z})=$ $\overline{\mathbf{c}}_{\mathbf{x}}, \ell g(\bar{y})=\theta$ such that: for each $\varphi(\bar{x}, \bar{y}, \bar{z})$ with $\ell g(\bar{x})=\ell g\left(\overline{\mathbf{d}}_{\mathbf{x}}\right), \ell g(\bar{y})=\theta, \ell g(\bar{z})=$ $\overline{\mathbf{c}}_{\mathbf{x}}$ (but $\varphi$ depends just on finitely many variables) we have:

- $\psi_{\varphi(\bar{x}, \bar{y}, \bar{z})}\left(\bar{x}, \bar{e}, \overline{\mathbf{c}}_{\mathbf{x}}\right) \in \operatorname{tp}\left(\overline{\mathbf{d}}_{\mathbf{x}}, \bar{e} \cup \overline{\mathbf{c}}_{\mathbf{x}}\right)$,

- $\psi_{\varphi(\bar{x}, \bar{y}, \bar{z})}\left(\bar{x}, \bar{e}, \overline{\mathbf{c}}_{\mathbf{x}}\right) \vdash\left\{\varphi\left(\bar{x}, \bar{b}, \overline{\mathbf{c}}_{\mathbf{x}}\right)^{\mathbf{t}}: \bar{b} \in{ }^{\ell g(\bar{y})} M\right.$ and $\mathbf{t} \in\{0,1\}$ such that $\left.\mathfrak{C} \models \varphi\left[\bar{d}_{\mathbf{x}}, \bar{b}, \overline{\mathbf{c}}_{\mathbf{x}}\right]^{\mathbf{t}}\right\}$

- $\operatorname{tp}\left(\bar{e}, M_{\mathbf{x}} \cup \overline{\mathbf{c}}_{\mathbf{x}} \cup \overline{\mathbf{d}}_{\mathbf{x}}\right)$ is finitely satisfiable in $M_{\mathbf{x}}$.

Proof. By 2.15 and 2.20 and compactness.

On strongly/strongly ${ }^{2}$ dependent theories see [Sh:863].

Remark 2.22. 1) If $T$ is strongly dependent then in the previous claim 2.20(1),

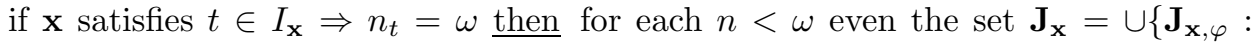
$\varphi(\bar{x}, \bar{y}, \bar{z}) \in \mathbb{L}\left(\tau_{T}\right)$ as there and $\left.\ell g(\bar{z})<n\right\}$ is finite.

2) If $Y$ is strongly dependent ${ }^{2}$ then above we can allow $n=\omega$.

3) The proofs are similar. 
We now turn to exact saturation. We first prove Theorem 2.2 second we prove in 2.23 that some independent $T$ 's satisfies it, even give a sufficient criterion. Third, we give a sufficient condition for $T$ to satisfy the theorem - in 2.26- the existence of a stable indiscernible set (2.24).

Proof. Proof of 2.2, The Singular Exact Saturation Lemma

Let $\theta=|T|$. As $M$ is not $\kappa^{+}$-saturated, there are $A \subseteq M$ of cardinality $\leq \kappa$ and $p \in \mathbf{S}^{1}(A)$ omitted by $M$. Let $d \in \mathfrak{C}$ realize $p$. By Theorem 2.4 there is $\overline{\mathbf{c}} \in{ }^{\theta \geq} \mathfrak{C}$ as there for (d), so in particular such that $\operatorname{tp}(\overline{\mathbf{c}}, M)$ does not split over some $N \prec M$ of cardinality $<\kappa$. Let $\left\langle B_{i}: i<\operatorname{cf}(\kappa)\right\rangle$ be a $\subseteq$-increasing sequence of sets with union $N \cup A$ such that $i<\operatorname{cf}(\kappa) \Rightarrow\left|B_{i}\right|<\kappa$ and $N \subseteq B_{0}$. Now we choose $A_{i} \subseteq M$ by induction on $i<\operatorname{cf}(\kappa)$ such that $A_{i}$ is of cardinality $\leq \theta$ and $\operatorname{tp}\left(d, A_{i} \cup \overline{\mathbf{c}}\right) \vdash \operatorname{tp}\left(d, B_{i}^{+}\right)$where $B_{i}^{+}:=B_{i} \cup \bigcup\left\{A_{j}: j<i\right\}$.

The choice is possible as $\left|B_{i}^{+}\right|<\kappa$ by 2.4, i.e. by the choice of $\overline{\mathbf{c}}$. Next we can find $A_{\kappa} \subseteq M$ of cardinality $\leq \theta$ such that $\operatorname{tp}\left(d, A_{\kappa} \cup \overline{\mathbf{c}}\right) \vdash \operatorname{tp}\left(d, \bigcup_{i<\kappa} A_{i}\right)$, possible as $\left|\bigcup_{i<\operatorname{cf}(\kappa)} A_{i}\right| \leq \theta+\operatorname{cf}(\kappa)<\kappa$. Let $B^{+}=\cup\left\{B_{i}^{+}: i<\operatorname{cf}(\kappa)\right\} \cup A_{\kappa}$ so $\left|B^{+}\right|=\kappa$ and we ask the question:

$\odot$ is there an elementary mapping $f$ (or automorphism of $\mathfrak{C}$ ) such that $f\left\lceil B^{+}\right.$ is the identity and $f(\overline{\mathbf{c}}) \in{ }^{\theta} M$ ?

If yes, then let $d^{\prime} \in M$ realize $f\left(\operatorname{tp}\left(d, A_{\kappa} \cup \overline{\mathbf{c}}\right) \in \mathbf{S}\left(A_{\kappa} \cup f(\overline{\mathbf{c}})\right)\right.$ hence there is an elementary mapping $g$ satisfying $g\left\lceil A_{\kappa}=\operatorname{id}_{A_{\kappa}}=f\left\lceil A_{\kappa}, g(\overline{\mathbf{c}})=f(\overline{\mathbf{c}})\right.\right.$ and $g(d)=d^{\prime}$. Hence easily for each $i<\operatorname{cf}(\kappa)$ the sequence $\left\langle d^{\prime}\right\rangle^{\wedge} f(\overline{\mathbf{c}})$ realizes $f\left(\operatorname{tp}\left(\langle d\rangle^{\wedge} \overline{\mathbf{c}}, A_{i}\right)\right)$ hence it realizes also $\left.f\left(\operatorname{tp}\left(\langle d\rangle^{\wedge} \overline{\mathbf{c}}, B_{i}^{+}\right)\right)\right)$; so $d^{\prime}$ realizes $f\left(\operatorname{tp}\left(d, B_{i}^{+}\right)\right)$. But as $B_{i}^{+}$ increases with $i$ it realizes $f\left(\operatorname{tp}\left(d, \cup\left\{B_{i}^{+}: i<\operatorname{cf}(\kappa)\right\}\right)\right.$, but $A \subseteq \cup\left\{B_{i}: i<\right.$ $\operatorname{cf}(\kappa)\} \subseteq \cup\left\{B_{i}^{+}: i<\kappa\right\}$ hence $d^{\prime}$ realizes $\operatorname{tp}(d, A)$, but $d^{\prime} \in M$ contradicting the choice of $p, A, d$.

Let $B_{i}^{*}=B_{i}^{+} \cup A_{\kappa}$ hence $\left\langle B_{i}^{*}: i<\operatorname{cf}(\kappa)\right\rangle$ is $\subseteq$-increasing with union $B^{+}$and $\left|B_{i}\right|<\kappa$ for $i<\operatorname{cf}(\kappa)$. So the answer to the question is no, which gives clauses (a),(b) of the desired conclusion. As for clause (c), we choose $\overline{\mathbf{c}}_{\varepsilon}$ by induction on $\varepsilon<\kappa$ such that:

(*) (a) $\quad \overline{\mathbf{c}}_{\varepsilon} \in{ }^{\theta} M$,

(b) $\overline{\mathbf{c}}_{\varepsilon}$ realizes $\operatorname{tp}\left(\overline{\mathbf{c}}, \cup\left\{\overline{\mathbf{c}}_{\zeta}: \zeta<\varepsilon\right\} \cup N\right)$,

(c) for even $\varepsilon$, if possible $\overline{\mathbf{c}}_{\varepsilon}$ does not realize $\operatorname{tp}\left(\overline{\mathbf{c}}, N \cup B^{+}\right)$hence for some $\alpha=\alpha_{\varepsilon}<\operatorname{cf}(\kappa), \overline{\mathbf{c}}_{\varepsilon}$ does not realize $\operatorname{tp}\left(\overline{\mathbf{c}}, B_{\alpha}^{*}\right)$,

(d) for even $\varepsilon$, if $\alpha_{\varepsilon}$ is well defined, it is minimal,

(e) for odd $\varepsilon, \alpha_{\varepsilon}=\alpha_{\varepsilon-1}$ and $\overline{\mathbf{c}}_{\varepsilon}$ realizes $\operatorname{tp}\left(\overline{\mathbf{c}}, B_{\alpha}^{*}\right)$ or even

$$
\operatorname{tp}\left(\overline{\mathbf{c}}, \cup\left\{\overline{\mathbf{c}}_{\zeta}: \zeta<\varepsilon\right\} \cup N \cup B_{\alpha_{\varepsilon}}^{*}\right) .
$$

There is no problem in carrying the induction, by $1.2(2)$ the sequence $\left\langle\overline{\mathbf{c}}_{\varepsilon}: \varepsilon<\kappa\right\rangle$ is indiscernible over $N$. Also obviously the sequence $\left\langle\alpha_{\varepsilon}: \varepsilon\langle\kappa\rangle\right.$ is non-decreasing; if $\alpha_{*}=\cup\left\{\alpha_{\varepsilon}: \varepsilon<\kappa\right\}$ is equal to $\operatorname{cf}(\kappa)$ we are done; that is letting $\overline{\mathbf{c}}_{\kappa}$ from ${ }^{\theta} M$ be such that $\left\langle\overline{\mathbf{c}}_{\varepsilon}: \varepsilon \leq \kappa\right\rangle$ is an indiscernible sequence over $N$, necessarily $\overline{\mathbf{c}}_{\kappa}$ realizes $\operatorname{tp}\left(\overline{\mathbf{c}}_{1}, N \cup B_{i}^{*}\right)$ for each $i<\kappa$ hence realizes $\operatorname{tp}\left(\overline{\mathbf{c}}, B^{+}\right)$contradiction to "๑ fails". But the non-existence of $\overline{\mathbf{c}}_{\kappa}$ and the properties of $\left\langle\overline{\mathbf{c}}_{i}: i\langle\kappa\rangle\right.$ are as promised in clause (c) of the Lemma, so we are indeed done. 
Otherwise, for each $\varepsilon<\kappa$ there is a formula $\varphi_{\varepsilon}\left(\bar{x}, \bar{e}_{\varepsilon}\right) \in \operatorname{tp}\left(\overline{\mathbf{c}}, B_{2 \varepsilon}^{*}\right) \subseteq \operatorname{tp}\left(\overline{\mathbf{c}}, B_{\beta}^{*}\right)$ with $\ell g(\bar{x})=\ell g(\overline{\mathbf{c}})$ such that $\mathfrak{C} \models \neg \varphi_{\varepsilon}\left[\overline{\mathbf{c}}, \bar{e}_{2 \varepsilon}\right]$, but by clause (e) of $(*)$ necessarily $\mathfrak{C} \models \varphi_{\varepsilon}\left[\overline{\mathbf{c}}_{2 \varepsilon+1}, \bar{e}_{2 \varepsilon}\right]$. As $\kappa>\left|B_{\beta}^{*}\right|+|T|$ for some formula $\varphi(\bar{x}, \bar{e})$ we have $\{\varepsilon<\kappa$ : $\left.\varphi_{\varepsilon}\left(\bar{x}, \bar{e}_{\varepsilon}\right)=\varphi\left(\bar{x}, \bar{e}_{\varepsilon}\right)\right\}$ is infinite. But this contradicts $T$ being dependent, so we are done proving Clause (c).

Clause $(c)^{+}$, that is "moreover, there is an ultrafilter $\mathscr{D}$ on $N$ " follows when we use the version of mxK from 2.13 $2 \mathrm{~A}$ ) or 2.33-2.35 below.

We may have hoped that 2.2 characterize being dependent, but this is not so. Clarification when this property (characterization of exactly $\kappa$-saturated $\kappa>$ $\operatorname{cf}(\kappa)>|T|$, as in 2.2) holds is given by:

Example 2.23. 1) There is an independent $T$ such that: if $T$ has an exactly $\kappa$-saturated model then $\kappa$ is regular. In fact, this is a sufficient condition.

2) The same holds for exactly $\kappa$-compact, $\kappa>\aleph_{0}$.

Proof. We use $T$ which satisfies "The Chang Trick" from his proof of his two cardinal theorem $\left(\aleph_{1}, \aleph_{0}\right) \rightarrow\left(\lambda^{+}, \lambda\right)$ when $\lambda=\lambda^{<\lambda}$; the use is not an incident, he uses such $T$ to overcome a related problem in his proof.

The condition is:

$\circledast$ for some predicate $R(x, y) \in \tau_{T}$ written $x R y$ (or just a formula $\varphi_{*}(x, y) \in$ $\left.\mathbb{L}\left(\tau_{T}\right)\right)$ we have13:

(a) the empty set can be coded, that is $\exists y \forall x(\neg x R y)$,

(b) we can add to a coded set one element, that is $(\forall x, y)\left(\exists y_{1}\right)\left(\forall x_{1}\right)\left[x_{1} R y_{1} \equiv\right.$ $\left.\left(x_{1} R y \vee x_{1}=x\right)\right]$.

Note: for any model $M$, if $R \notin \tau_{M}, M$ an infinite model, let $\left\langle u_{b}: b \in M\right\rangle$ list the finite subsets of $M$, and we expand $M$ to $M^{+}$by choosing $R^{\mu^{+}}=\left\{(a, b): a \in u_{b}\right.$ and $b \in M\}$, then $\operatorname{Th}\left(M^{+}\right)$is as required.

So assume $\kappa=\Sigma\left\{\kappa_{i}: i<\operatorname{cf}(\kappa)\right\}, \kappa_{i}<\kappa_{j}<\kappa$ for $i<j<\operatorname{cf}(\kappa), \operatorname{cf}(\kappa)<\kappa$ and $M$ is $\kappa$-saturated. Let $A \subseteq M,|A|=\kappa$ and $p \in \mathbf{S}(A, M)$. Let $A=\cup\left\{A_{i}: i<\right.$ $\operatorname{cf}(\kappa)\},\left|A_{i}\right| \leq \kappa_{i}, A_{i}$ increasing with $i$. Let $c_{i}$ realize $p\left\lceil A_{i}\right.$.

By induction on $i$, we choose $b_{i} \in M$ which realizes the type

$$
\begin{array}{ll}
p_{i}(y)=\left\{c_{j} R y: j<\operatorname{cf}(\kappa) \text { and } j \geq i\right\} \quad & \cup\left\{(\forall x)\left(x R y \rightarrow x R b_{j}\right): j<i\right\} \\
& \cup\left\{(\forall x)(x R y \rightarrow \varphi(x, \bar{a})): \varphi(\bar{x}, \bar{a}) \in p\left\lceil A_{i}\right\} .\right.
\end{array}
$$

Arriving to the $i$-th stage by $\circledast$ and the induction hypothesis on $i, p_{i}(y)$ is finitely satisfiable in $M$.

[Why? Let $p^{\prime}(y) \subseteq p_{i}(y)$ be finite so it has the form $\left\{c_{j} R y: j \in u\right\} \cup\{(\forall x)(x R y \rightarrow$ $\left.\left.x R b_{j}\right): j \in v\right\} \cup\left\{(\forall x)\left(x R y \rightarrow \varphi_{\ell}\left(x, \bar{a}_{\ell}\right)\right): \ell<n\right\}$ where $u \subseteq[i, \operatorname{cf}(\kappa))$ is finite, $v \subseteq i$ is finite and $\varphi_{\ell}\left(\bar{x}, \bar{a}_{\ell}\right) \in p\left\lceil A_{i}\right.$ for $\ell<n$. By $\circledast$ we can find $c \in M$ such that $M \models(\forall x)\left(x R c \equiv\left(\bigvee_{j \in v} x=b_{j}\right)\right.$, thus $c$ realizes $\left.\left.p^{\prime}(y)\right)\right]$.

But $\left|p_{i}\right|<\kappa$ so we can choose $b_{i}$.

Now $\left\{x R b_{i}: i<\operatorname{cf}(\kappa)\right\}$ is a set of formulas finitely satisfiable in $M$ of cardinality $<\kappa$ and any element realizing it realizes $p$.

\footnotetext{
$13_{\text {instead }(\mathrm{a})+(\mathrm{b}) \text { we can have }}$

$(a)^{\prime} \quad\left(\forall x_{0}, \ldots, x_{n-1}\right)(\forall z)\left(z R y=\bigvee_{\ell<n} z=x_{\ell}\right)$ for every $n$
} 
A weak complement to 2.4 is 2.26 but first recall:

Definition 2.24. [ $T$ not necessarily dependent].

1) $\mathbf{I} \subseteq{ }^{\alpha} \mathfrak{C}$ is a stable indiscernible set when: $\mathbf{I}$ is an infinite indiscernible set and $\operatorname{Av}(\mathbf{I}, \mathfrak{C})$ is well defined, i.e. for any $\varphi(\bar{x}, \bar{y}), \ell g(\bar{x})=\alpha$ and $\bar{b} \in{ }^{\ell g(\bar{y})} \mathfrak{C}$ either $\varphi(\mathbf{I}, \bar{b})$ or $\neg \varphi(\mathbf{I}, \bar{b})$ is finite.

2) $\mathbf{I}$ is a dependent indiscernible sequence when: $I$ is a linear order and $\mathbf{I}=\left\langle\bar{a}_{t}\right.$ : $t \in I\rangle$ is an indiscernible sequence and for every formula $\varphi(\bar{x}, \bar{b}), \ell g(\bar{x})=\ell g\left(\bar{a}_{t}\right)$, there is a convex equivalent relation $E$ on $I$ with finitely many equivalence classes such that $s E t \Rightarrow \varphi\left[\bar{a}_{t}, \bar{b}\right] \equiv \varphi\left[\bar{a}_{s}, \bar{b}\right]$.

Fact 2.25. If $T$ is dependent, $\mathbf{I} \subseteq{ }^{\alpha} \mathfrak{C}$ is a stable indiscernible set $\underline{\text { iff }} \mathbf{I}$ is an infinite indiscernible set.

Proof. By [Sh:715, 1.28].

Claim 2.26. 1) Assume ( $T$ is dependent and) there is an infinite indiscernible set $\mathbf{I} \subseteq \mathfrak{C}$. If $\kappa^{+}=2^{\kappa}$ and $\kappa>|T| \underline{\text { then }} T$ has an exactly $\kappa$-saturated model.

2) Assume ( $T$ not necessarily dependent), $\mathbf{I} \subseteq \mathfrak{C}$ is a stable indiscernible set. Then the conclusion of part (1) holds.

3) In parts (1),(2) the conclusion holds for $T$ if the assumption holds for $T^{\mathrm{eq}}$.

Remark 2.27. 1) Of course, trivially if for some non-zero ordinal $\alpha$ there is an infinite indiscernible set $\mathbf{I} \subseteq{ }^{\alpha} \mathfrak{C}$ then for some $i<\alpha,\left\{(\bar{a})_{i}: \bar{a} \in \mathbf{I}\right\}$ is an infinite indiscernible set.

2) But we could use below indiscernible set $\mathbf{I} \subseteq{ }^{\alpha} \mathfrak{C}$.

3) On indiscernible sets for $T$ dependent see [Sh:715, §1], we use it freely.

4) Of course: if $\left\{\bar{a}_{t}: t \in I\right\} \subseteq{ }^{\alpha} \mathfrak{C}$ is an infinite indiscernible set/a stable indiscernible set, $u \subseteq \alpha$ and $\left\langle\bar{a}_{t}\lceil u: t \in I\rangle\right.$ is not constant then $\left\{\bar{a}_{t}\lceil u: t \in I\} \subseteq{ }^{u} \mathfrak{C}\right.$ is an infinite indiscernible set/a stable indiscernible set. So using singletons in 2.26 is not a loss.

5) Recall that if $I$ is a linear order and $I_{1}, I_{2} \subseteq I$ are infinite, $\bar{a}_{t} \in{ }^{\beta} \mathfrak{C}$ for $t \in I$ and $\mathbf{I}=\left\langle\bar{a}_{\gamma}: t \in I\right\rangle$ is an indiscernible sequence then $\mathbf{I} \uparrow I_{1}$ is stable iff $\mathbf{I}\left\lceil I_{2}\right.$ is stable.

This is easy.

Proof. 1) By part (2) (and 2.25).

2) Let $\mathbf{I}=\left\{a_{\alpha}: \alpha<\kappa\right\} \subseteq \mathfrak{C}$ be an infinite stable indiscernible set. Now easily

$\odot_{1}$ for any $\bar{b} \in{ }^{\omega>} \mathfrak{C}$ for some $\mathbf{J} \in[\mathbf{I}]^{\leq|T|}$, the set $\mathbf{I} \backslash \mathbf{J}$ is an indiscernible set over $\bar{b} \cup \mathbf{J}$.

[Why? By the definition but we elaborate. First prove that for any $n$ and $\varphi=$ $\varphi\left(\bar{x}_{0}, \ldots, \bar{x}_{n-1}, \bar{b}^{\prime}\right)$ for some finite $\mathbf{J}_{\varphi}$ we have $\mathfrak{C} \models " \varphi\left[a_{0}, \ldots, a_{n-1}, \bar{b}^{\prime}\right] \equiv \varphi\left[a_{0}^{\prime}, \ldots, a_{n-1}^{\prime}, \bar{b}^{\prime}\right]$ " when $a_{0}, \ldots, a_{n-1}, a_{0}^{\prime}, \ldots, a_{n-1}^{\prime} \in \mathbf{I} \backslash \mathbf{J}_{\varphi}$ with no repetitions. Second, use transitivity of equivalence to show it suffices that $a_{0}, \ldots, a_{n-1} \in \mathbf{I} \backslash \mathbf{J}_{\varphi}$ with no repetition and $a_{0}^{\prime}, \ldots, a_{n-1}^{\prime} \in \mathbf{I} \backslash \mathbf{J}_{\varphi}$ with no repetitions. Lastly, we choose $\mathbf{J}_{k} \subseteq \mathbf{I}$ by induction on $k$ such that $\left\|\mathbf{J}_{k}\right\| \leq|T|, m<k \Rightarrow \mathbf{J}_{m} \subseteq \mathbf{J}_{k} \subseteq \mathbf{I}$ and if $k=m+1$ and $\varphi=\varphi\left(\bar{x}_{0}, \ldots, \bar{x}_{n-1}, \bar{b}^{\prime}\right), \bar{b}^{\prime} \subseteq\left(\cup \mathbf{J}_{m}\right) \cup \bar{b}$ then we can above choose $\mathbf{J}_{\varphi} \subseteq \mathbf{J}_{k}$. Now $\bigcup_{k} \mathbf{J}_{k}$ is as required.]

$\odot_{2}$ the following conditions on $\bar{b} \in{ }^{\omega>} \mathfrak{C}$ are equivalent:

(a) $\operatorname{tp}(\bar{b}, \mathbf{I}), \operatorname{Av}(\mathbf{I}, \mathbf{I})$ are weakly orthogonal, 
(b) for some $\mathbf{J} \in[\mathbf{I}] \leq|T|$ we have $\operatorname{tp}(\bar{b}, \mathbf{J}) \vdash \operatorname{tp}(\bar{b}, \mathbf{I})$.

[Why? Easy using $\odot_{1}$.]

$\odot_{3}$ let $\mathbf{D}=\mathbf{D}_{\mathbf{I}}=\left\{p \in \mathbf{S}^{<\omega}(\mathbf{I}): p\right.$ weakly orthogonal to $\left.\operatorname{Av}(\mathbf{I}, \mathbf{I})\right\}$.

We define

$\odot_{4}$ we say $A$ is a $\mathbf{D}$-set if $\bar{a} \in{ }^{\omega>} A \Rightarrow \operatorname{tp}(\bar{a}, \mathbf{I}) \in \mathbf{D}_{\mathbf{I}}$

$\odot_{5}$ if $\mathbf{I} \subseteq A$ we let $\mathbf{S}_{\mathbf{D}}^{m}(A)=\{\operatorname{tp}(\bar{b}, A): A \cup \bar{b}$ is a $\mathbf{D}$-set and $\ell g(\bar{b})=m\}$.

We note

$\odot_{6}(a) \quad$ if $A$ is a $\mathbf{D}$-set and $\mathbf{I} \subseteq A$ then $\operatorname{Av}(\mathbf{I}, \mathbf{I}) \vdash \operatorname{Av}(\mathbf{I}, A)$; hence if $A=|M|$, then $M$ is not $\kappa^{+}$-saturated,

(b) $A$ is a $\mathbf{D}$-set iff $A \cup \mathbf{I}$ is a $\mathbf{D}$-set.

$\odot_{7}$ If $A$ is a $\mathbf{D}$-set of cardinality $<\kappa$ and $p \in \mathbf{S}_{\mathbf{D}}^{m}(A \cup \mathbf{I}) \underline{\text { then }}$ for some $\mathbf{J} \subseteq \mathbf{I}$ of cardinality $\leq|A|+|T|$ we have $p\lceil(A \cup \mathbf{J}) \vdash p$.

[Why? By $\odot_{2}$ and $\odot_{6}$.

$\odot_{8}$ if $\left\langle A_{\alpha}: \alpha<\delta\right\rangle$ is an $\subseteq$-increasing sequence of $\mathbf{D}$-sets, then $A_{\delta}:=\cup\left\{A_{\alpha}\right.$ : $\alpha<\delta\}$ is a $\mathbf{D}$-set.

[Why? By the definition of a D-set.]

$\odot_{9}$ if $\left\langle A_{\alpha}: \alpha \leq \delta\right\rangle$ is $\subseteq$-increasing continuous sequence of $\mathbf{D}$-sets, $\mathbf{I} \subseteq A_{0}$ and $p \in \mathbf{S}^{m}\left(A_{\delta}\right)$ then $p \in \mathbf{S}_{\mathbf{D}}^{m}\left(A_{\delta}\right) \Leftrightarrow \bigwedge_{\alpha<\delta} p\left\lceil A_{\alpha} \in \mathbf{S}_{\mathbf{D}}^{m}\left(A_{\alpha}\right)\right.$.

[Why? By the definition of $\mathbf{S}_{\mathbf{D}}^{m}(-)$ and $\left.\odot_{8}.\right]$

Now comes a major point

$\odot_{10}$ if $A \subseteq \mathfrak{C}$ and $|A|<\kappa$ then we can find $\mathbf{I}_{1}$ such that: $\mathbf{I}_{1}$ is an indiscernible set, $\mathbf{I} \subseteq \mathbf{I}_{1},\left|\mathbf{I}_{1} \backslash \mathbf{I}\right| \leq|T|+|A|$ and $A$ is a $\mathbf{D}_{\mathbf{I}_{1}}$-set.

[Why? Let $\theta=|A|+|T|$ and we try by induction on $\alpha<\theta^{+}$to choose an element $a_{\alpha}$ of $\mathfrak{C}$ which realizes $\operatorname{Av}\left(\mathbf{I},\left\{a_{\beta}: \beta<\alpha\right\} \cup \mathbf{I}\right)$ but $\alpha$ is even iff $a_{\alpha}$ realizes $\operatorname{Av}\left(\mathbf{I}, \cup\left\{a_{\beta}\right.\right.$ : $\beta<\alpha\} \cup A \cup \mathbf{I})$. But if we succeed to carry the induction clearly $\mathbf{I}^{+}:=\mathbf{I} \cup\left\{a_{\alpha}\right.$ : $\left.\alpha<\theta^{+}\right\}$is an indiscernible set, and a stable one (recalling 2.27(5)) hence for some $\mathbf{J} \subseteq \mathbf{I}^{+}$of cardinality $\leq|A|+|T|$, also $\mathbf{I}^{+} \backslash \mathbf{J}$ is an indiscernible set over $A \cup \mathbf{J}$, but necessarily $\mathbf{J} \subseteq \mathbf{I} \cup\left\{a_{\beta}: \beta<\alpha\right\}$ for some $\alpha<\theta^{+}$, easy contradiction to the choice of the $a_{\alpha}$ 's.]

$\odot_{11}$ if $A_{1} \subseteq A_{2}$ are $\mathbf{D}_{\mathbf{I}}$-sets, $\left|A_{2}\right| \leq \kappa,\left|A_{1}\right|<\kappa$ and $p \in \mathbf{S}_{\mathbf{D}}^{m}\left(A_{1} \cup \mathbf{I}\right)$ then there is $q \in \mathbf{S}_{\mathbf{D}}^{m}\left(A_{2} \cup \mathbf{I}\right)$ extending $p$.

[Why $\odot_{11}$ ? By $\odot_{9}$ without loss of generality $A_{2}=A_{1} \cup\{b\}$, so $\left|A_{2}\right|<\kappa$. We can find $\bar{c}$ realizing $p(\bar{y})$ and let $A=A_{1} \cup\{b\} \cup \bar{c}=A_{2} \cup \bar{c}$. So by $\odot_{10}$ there is $\mathbf{I}^{+}$such that: $\mathbf{I}^{+}$is an indiscernible set, $\mathbf{I} \subseteq \mathbf{I}^{+},\left|\mathbf{I}^{+} \backslash \mathbf{I}\right| \leq \theta:=|A|+|T|=\left|A_{1}\right|+|T|<\kappa$ and $A$ is

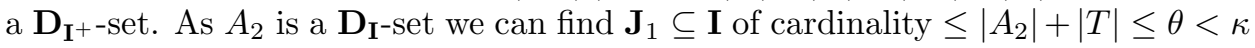

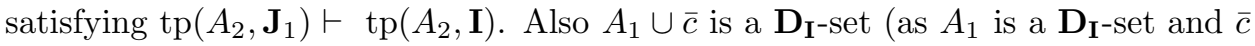
realizes $\left.p(\bar{y}) \in \mathbf{S}_{\mathbf{D}}^{m}\left(A_{1} \cup \mathbf{I}\right)\right)$ hence there is $\mathbf{J}_{2} \subseteq \mathbf{I}$ of cardinality $\leq\left|A_{1} \cup \bar{c}\right|+|T|=$ $\theta<\kappa$ such that $\operatorname{tp}\left(A_{1} \cup \bar{c}, \mathbf{J}_{1}\right) \vdash \operatorname{tp}\left(A_{1} \cup \bar{c}, \mathbf{I}\right)$. Lastly, as $A$ is a $\mathbf{D}_{\mathbf{I}^{+}-\text {set there is }}$ $\mathbf{J}_{3} \subseteq \mathbf{I}^{+}$of cardinality $\leq|A|+|T|=\theta$ such that $\operatorname{tp}\left(A, \mathbf{J}_{3}\right) \vdash \operatorname{tp}\left(A, \mathbf{I}^{+}\right)$. 
As tp $\left(A_{2}, \mathbf{J}_{1}\right) \vdash \operatorname{tp}\left(A_{2}, \mathbf{I}\right)$ necessarily $\operatorname{tp}\left(A_{2}, \mathbf{J}_{1}\right) \vdash \operatorname{tp}\left(A_{2}, \mathbf{I}^{+}\right)$. Similarly $\operatorname{tp}\left(A_{1} \cup\right.$ $\left.\bar{c}, \mathbf{J}_{2}\right) \vdash \operatorname{tp}\left(A_{2} \cup \bar{c}, \mathbf{I}^{+}\right)$. By cardinality considerations there is a permutation $h$ of $\mathbf{I}^{+}$which is the identity on $\mathbf{J}_{1}, \mathbf{J}_{2}$ and $\mathbf{J}_{3} \cap \mathbf{I}$ and maps $\mathbf{J}_{3} \backslash \mathbf{I}$ into $\mathbf{I}$. As $\mathbf{I}^{+}$is an indiscernible set, $h$ is an elementary mapping (of $\mathfrak{C}$ ). As $h \uparrow \mathbf{J}_{1}$ is the identity and $\operatorname{tp}\left(A_{2}, \mathbf{J}_{1}\right) \vdash \operatorname{tp}\left(A_{2}, \mathbf{I}^{+}\right)$, see above also $h \cup \operatorname{id}_{A_{2}}$ is an elementary mapping hence there is an automorphism $g$ of $\mathfrak{C}$ extending $h \cup \operatorname{id}_{A_{2}}$. As $\operatorname{tp}\left(A_{1} \cup \bar{c}, \mathbf{J}_{2}\right) \vdash$ $\operatorname{tp}\left(A_{1} \cup \bar{c}, \mathbf{I}^{+}\right)$and $h\left\lceil\mathbf{J}_{2}=\operatorname{id}_{\mathbf{J}_{2}}, h\left\lceil A_{1}=\operatorname{id}_{A_{1}}\left(\right.\right.\right.$ recalling $\left.A_{1} \subseteq A_{2}\right)$ and $h\left(\mathbf{I}^{+}\right)=\mathbf{I}^{+}$ necessarily $g(\bar{c})$ realizes $\operatorname{tp}\left(\bar{c}, A_{1} \cup \mathbf{I}^{+}\right)$hence it realizes $\operatorname{tp}\left(\bar{c}, A_{1} \cup \mathbf{I}\right)$ which is equal to $p(\bar{y})$. Also $\operatorname{tp}\left(A_{2} \cup \bar{c}, \mathbf{J}_{3}\right) \vdash \operatorname{tp}\left(A_{2} \cup \bar{c}, \mathbf{I}^{+}\right)$hence $\operatorname{tp}\left(A_{2} \cup g(\bar{c}), h\left(\mathbf{J}_{3}\right)\right) \vdash \operatorname{tp}\left(A_{2} \cup\right.$ $\left.h(\bar{c}), \mathbf{I}^{+}\right)$hence $\operatorname{tp}\left(A_{2} \cup g(\bar{c}), h\left(\mathbf{J}_{3}\right)\right) \vdash \operatorname{tp}\left(A_{2} \cup g(\bar{c}), \mathbf{I}\right)$, but $h\left(\mathbf{J}_{3}\right) \subseteq \mathbf{I}$. So $A_{2} \cup g(\bar{c})$ is a $\mathbf{D}_{\mathbf{I}}$-set hence $q(\bar{y})=: \operatorname{tp}\left(g(\bar{c}), A_{2} \cup \mathbf{I}\right)$ belongs to $\mathbf{S}_{\mathbf{D}_{\mathbf{I}}}^{m}\left(A_{2} \cup \mathbf{I}\right)$ so is as required.]

As $2^{\kappa}=\kappa^{+}$, by $\odot_{9}+\odot_{11}$ there is $M \supseteq \mathbf{I}$ of cardinality $\kappa^{+}$which is $\kappa$-saturated and is a $\mathbf{D}$-set hence by $\odot_{6}$ is not $\kappa^{+}$-saturated.

3) Should be clear.

Observation 2.28. (Any complete first order $T$ )

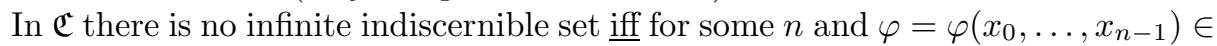
$\mathbb{L}\left(\tau_{T}\right), \varphi$ is connected and anti-symmetric i.e. if $a_{0}, \ldots, a_{n-1} \in \mathfrak{C}$ with no repetitions then for some permutations $\pi_{1}, \pi_{2}$ of $\{0, \ldots, n-1\}$ we have

$$
\mathfrak{C} \models \varphi\left[a_{\pi_{1}(0)}, \ldots, a_{\pi_{1}(n-1)}\right] \wedge \neg \varphi\left[a_{\pi_{2}(a)}, \ldots, a_{\pi_{2}\left(a_{n-1}\right)}\right] .
$$

Remark 2.29. 1) The second condition is related to the property (E) of complete first order theories of Ehrenfeucht [Ehr57] which says that the condition holds for some infinite set.

2) Note that $\mathfrak{C}$ may have no infinite indiscernible set but $\mathfrak{C}^{\mathrm{eq}}$ has.

Proof. The implication $\Leftarrow$ is obvious.

So assume the first statement. For $\alpha \leq \omega$ and $\Delta \subseteq \Delta_{*}:=\{\varphi(\bar{x}): \varphi \in$ $\left.\mathbb{L}\left(\tau_{T}\right), \bar{x}=\left\langle x_{\ell}: \ell<n\right\rangle\right\}$ let $14 \Gamma_{\Delta}^{\alpha}=\left\{y_{k} \neq y_{\ell}: k<\ell<\alpha\right\} \cup\left\{\varphi\left(y_{k_{0}}, \ldots, y_{k_{n-1}}\right) \equiv\right.$ $\varphi\left(y_{\ell_{0}}, \ldots, y_{\ell_{n-1}}\right): n<\alpha, \varphi\left(x_{0}, \ldots, x_{n-1}\right) \in \mathbb{L}\left(\tau_{T}\right)$ and $k_{0}, \ldots, k_{n-1}<\alpha$ without repetitions and $\ell_{0}, \ldots, \ell_{n-1}<\alpha$ without repetitions $\}$. Easily $\Gamma_{\Delta_{*}}^{\omega}$ is not realized in $\mathfrak{C}$ by the present assumption and $\left\langle\Gamma_{\Delta}^{k}: \Delta\right.$ is a finite subset of $\Delta_{*}$ and $\left.k<\omega\right\rangle$ is $\subseteq$-increasing with $k$ and $\Delta$ with union $\Gamma_{\Delta_{*}}^{\omega}$. Hence for some finite $\Delta \subseteq \Delta_{*}$ and $k<\omega$, the set $\Gamma_{\Delta}^{k}$ is not realized in $\mathfrak{C}$.

Let $\left\langle\varphi_{i}\left(x_{0}, \ldots, x_{n_{i}-1}\right): i<i(*)\right\rangle$ list $\Delta$ so $i(*)<\omega$, so without loss of generality $n_{i}<k$ for $i<i(*)$. Lastly, we define $\varphi\left(y_{0}, \ldots, y_{k-1}\right)$, it says: if $\left\langle y_{\ell}: \ell<k\right\rangle$ is without repetitions and $i$ is minimal such that $\left\langle y_{\ell}: \ell<k\right\rangle$ is not a $\left\{\varphi_{i}\left(x_{0}, \ldots, x_{n_{i}-1}\right)\right\}$ indiscernible set then $\varphi_{i}\left(y_{0}, \ldots, y_{n_{i}-1}\right)$. Now check.

Question 2.30. 1) Is there a dependent $T$ such that even in $\mathfrak{C}^{\text {eq }}$ there is no infinite indiscernible set but some singular $\kappa$ of cofinality $>|T|$ there is an exactly $\kappa$ saturated model of $T$ ?

2) For a dependent theory $T$ characterize $\{\kappa: \kappa$ singular and $T$ has exactly $\kappa$ saturated models\}.

3) In both parts we may at least initially restrict ourselves to $\kappa$ strong limit of large enough cofinality such that $2^{\kappa}=\kappa^{+}$.

\footnotetext{
${ }^{14}$ yes: we use singletons $y$ 's.
} 
4) Try to eliminate the assumption " $2^{\kappa}=\kappa^{+}$" in 2.26 at least when $\kappa$ is strong limit of cofinality $>|T|$. A natural way is via $\mathscr{P}^{-}(n)$-diagrams (as in [Sh:C, Ch.XII] and even closer in Sh:234.

The following in a sense gives a spectrum for $\overline{\mathbf{d}} / M$.

Claim 2.31. For $\theta \geq|T|$, a model $M$ and sequence $\overline{\mathbf{d}} \in{ }^{\theta \geq \mathfrak{C}}$, there is a set $\Theta$ such that:

(a) $\Theta \subseteq \Theta_{*}:=\{\kappa: \kappa>\theta$ and $M$ is $\kappa$-saturated $\}$,

(b) $|\Theta| \leq \theta$

(c) if $\kappa \in \Theta$ and $\operatorname{cf}(\kappa)>\theta$ then there is $\mathbf{x} \in \operatorname{mxK}_{\kappa, \leq \theta}$ such that $M_{\mathbf{x}}=M, \overline{\mathbf{d}}_{\mathbf{x}}=$ $\overline{\mathbf{d}}$ and $\left|B_{\mathbf{x}}\right| \leq \theta+\sup (\Theta \cap \kappa)$,

(d) if $\theta<\kappa \in \Theta$ and $\operatorname{cf}(\kappa) \leq \theta$ then $\sup (\Theta \cap \kappa)=\kappa$.

Proof. Straight.

For each $\kappa \in \Theta^{\prime}:=\left\{\kappa^{\prime} \in \Theta_{*}: \kappa^{\prime}\right.$ has cofinality $\left.>\theta\right\}$ we can find $\mathbf{x}_{\kappa} \in \operatorname{mxK}_{\kappa, \leq \theta}$ such that $M_{\mathbf{x}}=M, \overline{\mathbf{d}}_{\mathbf{x}}=\overline{\mathbf{d}}$ and for $\kappa \in \Theta_{*} \backslash \Theta^{\prime}$ let $\Theta_{\kappa}$ be a cofinal subset of $\kappa$ of cardinality $\operatorname{cf}(\kappa) \leq \theta$. Let $f: \Theta^{\prime} \rightarrow$ Card be defined by $f(\kappa)=\left|B_{\mathbf{x}}\right|+\theta$. Note that $\Theta^{\prime}$ has a maximal member or $\Theta^{\prime}$ has a cofinal subset of cardinality $\leq \theta$. Now we shall choose $\Theta_{n}$ by induction on $n$ such that $\Theta_{n} \subseteq \Theta_{*},\left|\Theta_{n}\right| \leq \theta$ and $n=m+1 \Rightarrow$ $\Theta_{m} \subseteq \Theta_{n}$. Let $\Theta_{0}$ be a cofinal subset of $\Theta_{*}$ of cardinality $\leq \theta$, see above why possible. If $n=m+1$, let $\Theta_{n}=\left\{f(\kappa): \kappa \in \Theta_{m} \cap \Theta^{\prime}\right\} \cup\left\{\Theta_{\kappa}: \kappa \in \Theta_{m} \backslash \Theta^{\prime}\right\} \cup \Theta_{m}$. Now $\cup\left\{\Theta_{n}: n<\omega\right\}$ is as required.

2.31

Discussion 2.32. Note that $\mathbf{P}_{\mathbf{x}}$ in 2.4 is $\kappa$-directed, but in general it is not definable in $M_{\mathbf{x}}$ and even not definable in $\left(M_{\mathbf{x}}\right)_{\left[\overline{\mathbf{c}}_{\mathbf{x}}\right]}\left(\right.$ or $\left.M_{\left[B_{\mathbf{x}}+\mathbf{c}_{\mathbf{x}}\right]}\right)$ even by disjunction of types as it depends on $\overline{\mathbf{d}}_{\mathbf{x}}$. So we may consider $\mathbf{P}_{\mathbf{x}}^{\prime}=\mathbf{P}_{M, \overline{\mathbf{c}}_{\mathbf{x}}, \alpha_{\mathbf{x}}}=\left\{p: p \in \mathbf{S}^{\alpha(\mathbf{x})}\left(A \cup \overline{\mathbf{c}}_{\mathbf{x}}\right)\right.$ and $A \subseteq M$ has cardinality $<\kappa\}$ ordered as before. Now $\mathbf{P}_{\mathbf{x}}^{\prime}$ is partially ordered but it is not clear that it is $\kappa$-directed. Moreover $\left(M_{\mathbf{x}}\right)_{\left[\overline{\mathbf{c}}_{\mathbf{x}}\right]}$ is not $\kappa$-saturated, but is $\left(\mathbf{D}_{\mathbf{x}}, \kappa\right)$ - sequence homogeneous for suitable $\mathbf{D}_{\mathbf{x}}$ and $\mathbf{D}_{\mathbf{x}}$ is a good diagram (see e.g. [Sh:3]; see more in [Sh:950]). So we can consider the families of such D's, fixing ( $T$ and) $\theta$.

But we can define the order in the $\kappa$-saturated $\left(M_{\mathbf{x}}\right)_{\left[B_{\mathbf{x}}\right]}$ which is $\mathbb{L}_{\infty, \kappa}\left(\tau_{T}\right)$ equivalent to $\mathfrak{C}_{\left[B_{\mathbf{x}}\right]}$. In this model we have $\psi(\bar{x}, \bar{y}) \in \mathbb{L}_{\infty, \kappa}$ which is a partial order on the $\theta$-tuples, $\ell g(\bar{x})=\alpha_{\mathbf{x}}=\ell g(\bar{y})$.

However, in our case we know more. Letting $\Gamma=\mathbb{L}\left(\tau_{T}\right)$, if $\operatorname{cf}(\kappa)>\theta \geq|T|$ we know that we can find $\bar{\psi}=\left\langle\psi_{\varphi}\left(\bar{x}_{\varphi}, \bar{y}_{\varphi}, \bar{z}_{\varphi}\right): \varphi \in \Gamma\right\rangle$ and the order on the set of $\overline{\mathbf{e}}=\left(\ldots \bar{e}_{\varphi} \wedge \ldots\right)_{\varphi}$ such that in 2.15 we can choose $\psi=\psi_{\varphi}$ (easy, see 2.20(0)). If $\operatorname{cf}(\kappa)>2^{\theta}$, we can fix there also the type of $\overline{\mathbf{e}}$ over $C_{\mathbf{x}} \cup \overline{\mathbf{d}}_{\mathbf{x}}$.

So

$(*)_{1}$ let $\mathbf{I}=\{\bar{e}: \bar{e}$ as above $\}$, so $\mathbf{I}$ is type-definable in $\left(M_{\mathbf{x}}\right)_{\left[B_{\mathbf{x}}\right]}$.

$(*)_{2} p_{\overline{\mathbf{e}}}=\left\{\psi_{\varphi}\left(\bar{x}_{\varphi}, \overline{\mathbf{e}}, \overline{\mathbf{c}}_{\mathbf{x}}\right): \varphi \in \Gamma\right\}$ for $\overline{\mathbf{e}} \in \mathbf{I}$.

$(*)_{3} \quad(a) \quad \leq_{1}$ defined by $\overline{\mathbf{e}}_{1} \leq \overline{\mathbf{e}}_{2}$ if $p_{\overline{\mathbf{e}}_{2}} \vdash p^{\prime}$ for some $p^{\prime}$ such that $p_{\overline{\mathbf{e}}_{1}} \subseteq p^{\prime} \in \mathbf{S}\left(B_{\mathbf{x}} \cup \overline{\mathbf{e}}_{1}\right)$, 
(b) $\mathbf{R}$ is defined by $\mathbf{e}_{1} \operatorname{Re}_{2}$ iff $\psi_{\varphi}\left(\bar{x}, \bar{e}_{\varphi}, \bar{c}\right) \vdash p^{\prime} \cap\{\varphi(\bar{x}, \bar{b}, \bar{c})$ :

$\left.\bar{b} \subseteq \operatorname{Rang}\left(\overline{\mathbf{e}}_{1} \cup B_{\mathbf{x}}\right)\right\}$ for each $\varphi \in \Gamma$ where $p^{\prime}$ is as above.

There are other variants, we intend to return to this.

We now consider some variants of the main Definition 2.6.

Definition 2.33. 1) In Definition 2.6 we add and define $K_{\ell}, K_{\lambda, \kappa, \theta}^{\ell}$, etc., also for $\ell=2,3$ by replacing Clause $(f)_{1}=(f)$ by $(f)_{\ell}$ where:

$(f)_{2}$ if $\ell=2$ then $\operatorname{tp}\left(\bar{c}_{t}, A \cup\left\{\bar{c}_{s, m}: s<_{I} t, m<n_{s}\right\}\right)$ is finitely satisfiable in $B$

$(f)_{3}$ if $\ell=3$ then for some endless indiscernible sequence $\overline{\mathbf{b}}_{t}=\left\langle\bar{b}_{t, r}: r \in J_{t}\right\rangle$ of sequences from $B$, the sequence $\bar{c}_{t, 0}$ realize: 15 the type $\operatorname{Av}\left(A \cup\left\{\bar{c}_{s, m}: s<_{I}\right.\right.$ $\left.\left.t, m<n_{t}\right\}, \overline{\mathbf{b}}_{t}\right)$.

2) We define $\operatorname{mxK}_{\lambda, \kappa, \theta}^{\ell}$ similarly.

Claim 2.34. 1) $K_{3} \subseteq K_{2} \subseteq K_{1}$.

2) If $\ell \in\{2,3\}$ and $\left\langle\mathbf{x}_{\alpha}: \alpha<\delta\right\rangle$ is $\leq_{1}$-increasing in $K_{\lambda, \kappa,<\theta}^{\ell}$ and $\delta<\operatorname{cf}(\theta), \delta<\operatorname{cf}(\kappa)$ $\underline{\text { then }} \mathbf{x}_{\delta}=\bigcup_{\alpha<\delta} \mathbf{x}_{\alpha}$ defined as in 2.7 belongs to $K_{\lambda, \kappa,<\theta}^{\ell}$ and is a $\leq_{1}$-lub of the sequence.

3) If $\ell=0,1,2,3$ and $\overline{\mathbf{d}} \in{ }^{\theta>C} \mathfrak{C}$ and $M$ is $\kappa$-saturated, $\operatorname{cf}(\kappa) \geq \theta$ then $\mathbf{x}=(M, \emptyset,<>$ $, \overline{\mathbf{d}}) \in K_{\kappa,<\theta}^{\ell}$.

4) Like 2.12 for $\ell=2,3$, i.e.: if $\operatorname{cf}(\theta)>|T|, \operatorname{cf}(\kappa)>\theta, \ell \leq 3$ and $\mathbf{x} \in K_{\lambda, \kappa,<\theta}^{\ell}$ then 16 for some $\mathbf{y}$ we have $\mathbf{x} \leq_{1} \mathbf{y} \in \operatorname{mxK}_{\lambda, \kappa,<\theta}^{\ell}$; so in $\operatorname{tp}\left(\mathbf{c}_{\mathbf{x}}, M_{\mathbf{y}}+\mathbf{c}_{\mathbf{x}}\right)$ we can get $\operatorname{tp}(\bar{c}, M)$ is finitely satisfiable in $\left.M_{\mathbf{y}}+\left(C_{\mathbf{y}} \backslash C_{\mathbf{x}}\right)\right)$.

5) If $\mathbf{x} \in K_{2}$ and $\bar{c} \in C_{\mathbf{x}} \underline{\text { then }} \operatorname{tp}\left(\bar{c}, A_{\mathbf{x}}\right)$ is finitely satisfiable in $B_{\mathbf{x}}$.

Proof. Similar to the proofs for $\ell=1$.

Claim 2.35. 1) In 2.14 we can deal with $K_{\lambda, \kappa,<\theta}^{\ell}, \ell=2,3$, i.e. if $\ell=2$ we should strengthen the assumption to " $q$ is finitely satisfiable in $B$ ".

2) If $\ell=3$ we should strengthen the assumption to $q=\operatorname{Av}\left(M_{\mathbf{x}} \cup C_{\mathbf{x}}, \mathbf{I}\right)$, I an endless indiscernible sequence of cardinality $<\kappa$.

3) In 2.15 we can deal with $\operatorname{mxK}_{\lambda, \kappa, \theta}^{2}$.

Proof. Similar to the proof for $\ell=1$.

\footnotetext{
$15_{\text {we may consider " }} \bar{c}_{t}$ realizes seeming this makes no difference.

${ }^{16}$ so if $\kappa=\operatorname{cf}(\kappa)>\theta \geq|T|, M$ is $\kappa$-saturated and $\overline{\mathbf{d}} \in \theta^{+}>\mathfrak{C}$ then for some $\overline{\mathbf{c}} \in \theta^{+}>\mathfrak{C}$ and $B \in[M]^{<\kappa}$ we have $(M, B, \overline{\mathbf{c}}, \overline{\mathbf{d}}) \in \mathrm{mxK}_{2}$.
} 


\section{§ 3. EXISTENCE OF STRICT TYPE DECOMPOSITIONS}

We here get a better decomposition, but at a price: using normal ultrafilters (so measurable or supercompact cardinals). Why is the decomposition from $\S 2$ not enough for our purposes? See 4.5 below.

Hypothesis 3.1. We assume $T$ is dependent, $\mathfrak{C}=\mathfrak{C}_{T}$ a monster; if not said otherwise, we assume (1) or just (2) where:

(1) (a) $\lambda=\kappa$ is a measurable cardinal,

(b) $\mathscr{D}$ is a normal ultrafilter on $I=\lambda$, so $I$ is a linear order,

(c) $M_{\alpha} \prec \mathfrak{C}$ is $\prec$-increasing, $\left\|M_{\alpha}\right\|<\lambda$ for $\alpha<\lambda$,

(d) $\quad M_{\lambda}=\bigcup_{\alpha<\lambda} M_{\alpha}$, by Clauses (a) + (c) + (e) necessarily $M$ is saturated,

(e) $\quad M_{\alpha}$ is $\left\|M_{\beta}\right\|^{+}$-saturated for $\beta<\alpha$,

(2) (a) $I$ is the following partial order, which is $(<\kappa)$-directed, and:

$(\alpha)$ set of elements $\left\{a \in[\lambda]^{<\kappa}: a \cap \kappa \in \kappa\right\}$ and

( $\beta) \quad s \leq_{I} t$ iff $s \subseteq t \wedge|s|<\min (\kappa \backslash t)$,

(b) $\mathscr{D}$ is a fine normal ultrafilter on $I$ and it follows that $\kappa$ is a measurable cardinal $\leq \lambda$,

(c) $M_{t} \prec \mathfrak{C},\left\|M_{t}\right\|<\kappa$ and $s<_{I} t \Rightarrow M_{s} \prec M_{t}$,

(d) $\quad M_{\lambda}=\cup\left\{M_{t}: t \in I\right\}$ (by (a)+(c)+(e), $M_{\lambda}$ is $\kappa$-saturated)

(e) if $s<_{I} t$ then $M_{t}$ is $\left\|M_{s}\right\|^{+}$-saturated.

Remark 3.2. 1) So in 3.1 we can define:

(A) like (2) without the normality and

(B) $(a)(b),(c),(d)$ of part (2).

2) Note that we have $(1) \Rightarrow(2) \Rightarrow(A)$ and $(2) \Rightarrow(B)$.

Notation 3.3. 1) In 3.1(1) let $\kappa_{I}(t):=t$ for $t \in I$, this notation is introduced only for having a uniform treatment of (1) and (2).

2) In $3.1(2)$ let $\kappa_{I}(t)=\operatorname{Min}(\kappa \backslash t)$ for $t \in I$.

Definition 3.4. [under 3.1(1) or (2) or alternatively (B) from 3.2 so these notions depend on $\left\langle M_{t}: t \in I\right\rangle$.]

1) For $\mathscr{U} \subseteq I$ (usually $\in \mathscr{D}$ ), so is a partial order, we say $\left\langle\bar{a}_{t}: t \in \mathscr{U}\right\rangle$ is indiscernible in $M_{\lambda}$ over $A$ when $(A \subseteq M$ and):

(a) $\ell g\left(\bar{a}_{t}\right)$ is constant, possibly infinite, and $\bar{a}_{t} \subseteq M$ for $t \in \mathscr{U}$,

(b) for each $n$ for some $p_{n}$ for every $t_{0}<_{I} \ldots<_{I} t_{n-1}$ from $\mathscr{U}$ we have $\operatorname{tp}\left(\bar{a}_{t_{0}}{ }^{\wedge} \ldots{ }^{\wedge} \bar{a}_{t_{n-1}}, A, M\right)=p_{n}$, the ninth paragraph of $\S 0$.

2) We say $\left\langle\bar{a}_{t}: t \in \mathscr{U}\right\rangle$ is fully indiscernible (in $M_{\lambda}$ ) over $A$ when Clauses (a),(b) above holds and

(c) if $s<_{I} t$ are from $\mathscr{U}$ then $\bar{a}_{s} \subseteq M_{t}$, recalling $\bar{M}$ is from 3.1

(d) if $s \in \mathscr{U}$ then recalling $\bar{M}$ is from $3 . \mathbb{1}^{7}$ the sequence $\left\langle a_{t}: t \in \mathscr{U} \cap I_{\geq s}\right\rangle$ is indiscernible over $M_{s} \cup A$ where, of course, $I_{\geq s}:=\left\{t \in I: s \leq_{I} t\right\}$.

\footnotetext{
${ }^{17}$ By normality (i.e. if (1) or (2) or (B) holds) then this follows.
} 
3) In parts $(1),(2)$ of the definition we say $k$-indiscernible when in Clause (b) we demand $n \leq k$.

We shall in Theorem 3.10 (and see 3.8) below prove the existence of:

Definition 3.5. 1) For an infinite linear order $J$ we say $\left\langle\left(\bar{c}_{t}, \bar{d}_{t}\right): t \in J\right\rangle$ is a strict $_{1}(\kappa,<\theta)$-decomposition over $(M, B)$ (and over $M$ means "for some $B \subseteq M$ of cardinality $\left.<\kappa^{\prime \prime}\right)$ when:

(a) $B \subseteq M$ is of cardinality $<\kappa$ and $M \prec \mathfrak{C}$ is $\kappa$-saturated, but if we write $<\aleph_{0}$ instead of $\kappa$ we mean $M \prec \mathfrak{C}$ and if we write 0 instead of $\kappa$ we replace $M$ by a set $\supseteq B$,

(b) $\alpha=\ell g\left(\bar{c}_{t}\right), \beta=\ell g\left(\bar{d}_{t}\right)$ are $<\theta$,

(c) if $t_{0}<_{J} \ldots<_{J} t_{n}$ then $\operatorname{tp}\left(\bar{c}_{t_{n}}, M+\bar{c}_{t_{0}}{ }^{\wedge} \bar{d}_{t_{0}}+\ldots+\bar{c}_{t_{n-1}}{ }^{\wedge} d_{t_{n-1}}\right)$ is increasing with $n \leq k$ and does not split over $B$,

(d) $\left\langle\left(\bar{c}_{t} \wedge \bar{d}_{t}: t \in J\right\rangle\right.$ is an indiscernible sequence over $M$,

(e) if $s<_{J} t$ then $\operatorname{tp}\left(\bar{d}_{t}, \bar{c}_{t}+\bar{d}_{s}\right) \vdash \operatorname{tp}\left(\bar{d}_{t}, \bigcup\left\{\bar{c}_{r}{ }^{\wedge} \bar{d}_{r}: r \leq_{J} s\right\} \cup \bigcup\left\{\bar{c}_{r}: r \in J\right\} \cup M\right)$,

(f) for every $A \subseteq M$ of cardinality $<\kappa$ for some $\bar{c}^{\wedge} \bar{d} \in{ }^{\alpha+\beta} M$, the sequence $\left\langle\bar{c}^{\wedge} \bar{d}\right\rangle^{\wedge}\left\langle\bar{c}_{t} \wedge \bar{d}_{t}: t \in J\right\rangle$ is an indiscernible sequence over $A$, so if $\kappa=0$ this is an empty demand.

2) We say strict $-1(\kappa, \theta)$-decomposition if (in part (1)) we omit Clauses (e) and (f). 3 ) We say $\operatorname{strict}_{0}(\kappa,<\theta)$-decomposition if we omit (f) and weaken (e) to (e) ${ }^{-}$, where

$(e)^{-}$if $s<_{I} t$ then $\operatorname{tp}\left(\bar{d}_{t}, \bar{c}_{t}+\bar{d}_{s}\right) \vdash \operatorname{tp}\left(\bar{d}_{t}, \cup\left\{\bar{c}_{r}{ }^{\wedge} \bar{d}_{r}: r \leq s\right\} \cup \bar{c}_{t} \cup M\right)$.

4) Notation:

- If $\theta=\sigma^{+}$instead of " $<\theta$ " we may write $\sigma$.

- If $\kappa=0$ then $M$ is replaced by a set $B$, if we write $<\aleph_{0}$ instead of $\kappa$ then $M$ is just a model.

- Strict $_{1}$ may be written strict.

A natural question about those notions of indiscernibility is about existence results. Now 3.6 is a well known set-theoretic existence and 3.8 is existence for dependent theories.

Fact 3.6. 1) If $A \subseteq \mathfrak{C},|A|<\kappa, \alpha<\kappa, \mathscr{U}_{1} \in \mathscr{D}$ and $\bar{a}_{t} \in{ }^{\alpha} \mathfrak{C}$ for $t \in \mathscr{U}_{1}$ then for some $\mathscr{U}_{2} \subseteq \mathscr{U}_{1}$ from $\mathscr{D}$ the sequence $\left\langle\bar{a}_{t}: t \in \mathscr{U}_{2}\right\rangle$ is indiscernible over $A$.

2) If in addition $\bar{a}_{t} \in{ }^{\alpha}\left(M_{\lambda}\right) \underline{\text { then }}$ we can add "fully indiscernible".

3) If $\iota \in\{-1,0,1\}$ and $\left\langle\left(\bar{c}_{t}, \bar{d}_{t}\right): t \in J\right\rangle$ is a $\operatorname{strict}_{\iota}(\kappa,<\theta)$-decomposition over $(M, B)$ and $M \subseteq B_{1} \subseteq B \underline{\text { then }}$ it is a $\operatorname{strict}_{\iota}(\kappa,<\theta)$-decomposition over $(M, B)$. Similarly we can replace $M$ by $M^{\prime}$ if $B_{1} \subseteq M^{\prime} \subseteq M$ and $M_{1}$ satisfies Clause (a) in Definition 3.5.

Proof. 1),2) By well known set theory (see Kanamori Magidor [KM78]).

Observation 3.7. 1) For some $\mathscr{U}_{*} \in \mathscr{D}$, for every $t \in I$ the model $M_{t}$ is $\kappa(t)$ saturated and $\kappa(t)>|T|$. 
2) If $M \prec \mathfrak{C}$ is $\kappa$-saturated, $J$ an infinite linear order $\theta \geq \aleph_{0}, B \in[M]^{<\kappa}$ and $\bar{c}_{t}=\langle\rangle=\bar{d}_{t}$ for $t \in J$ then $\left\langle\left(\bar{c}_{t}: \bar{d}_{t}\right): t \in J\right\rangle$ is a strict $_{\iota}(\kappa,<\theta)$-decomposition over $(M, B)$ for $\iota=1$ hence for $\iota \in\{-1,0\}$, too.

Proof. Obvious.

Theorem 3.8. Assume $\theta$ satisfies $\kappa>\theta \geq|T|$ and recall $\lambda \geq \kappa$. For every $\gamma(*)<\theta^{+}$and $\bar{d} \in \gamma^{(*)} \mathfrak{C}$ there are $B$ and $\left\langle\left(\bar{c}_{t}^{\omega}, \bar{d}_{t}^{\omega}\right): t \in \mathscr{U} \cup\{\lambda\}\right\rangle$ such that:

$\otimes(a) \quad \mathscr{U} \in \mathscr{D}$,

(b) $B \subseteq M_{\lambda},|B|<\kappa$,

(c) $\ell g\left(\bar{d}_{t}^{\omega}\right)=\gamma(*)+\theta \cdot \omega$,

(d) $\bar{d} \unlhd \bar{d}_{\lambda}^{\omega}$,

(e) $\mathbf{x}=\left(M_{\lambda}, B, \bar{c}_{\lambda}^{\omega}, \bar{d}_{\lambda}^{\omega}\right) \in \operatorname{mxK}_{\kappa, \theta}$,

(f) $\quad \bar{c}_{t}^{\omega \wedge} \bar{d}_{t}^{\omega} \subseteq M_{\lambda}$ realizes $\operatorname{tp}\left(\bar{c}_{\lambda}^{\omega \wedge} \bar{d}_{\lambda}^{\omega}, M_{t}\right)$ for $t \in \mathscr{U}$,

(g) $\left\langle\bar{c}_{t}^{\omega \wedge} \bar{d}_{t}^{\omega}: t \in \mathscr{U}\right\rangle$ is fully indiscernible (in $M_{\lambda}$ ) over $B$ and even over $B \cup \bar{c}_{\lambda}^{\omega} \cup \bar{d}_{\lambda}^{\omega}$, (see Definition 3.4(2)),

$(h)_{1} \quad$ if $t_{0}<_{I} \ldots<_{I} t_{m}<_{I} \ldots<_{I} t_{n}$ belongs to $\mathscr{U}$, so $m<n$ and possibly $m+1=n$; moreover possibly $0=m \underline{\text { then }}$ $\operatorname{tp}\left(\bar{d}_{t_{m+1}}^{\omega}, \bar{c}_{t_{m+1}}^{\omega}+\bar{c}_{t_{m+2}}^{\omega}+\ldots+\bar{c}_{t_{n}}^{\omega}+\overline{\bar{d}}_{t_{n}}^{\omega}\right) \vdash$ $\operatorname{tp}\left(\bar{d}_{t_{m+1}}^{\omega}, \bar{c}_{t_{0}}^{\omega}+\ldots+\bar{c}_{t_{m+1}}^{\omega}+\ldots+\bar{c}_{t_{n}}^{\omega}+\bar{d}_{t_{0}}^{\omega}+\ldots+\bar{d}_{t_{m}}^{\omega}+M_{t_{0}}\right)$,

$(h)_{2} \quad$ if $s<_{I}$ t are from $\mathscr{U}$ then $\bar{c}_{s}^{\omega \wedge} \bar{d}_{s}^{\omega}$ is from $M_{t}$, (actually follows from clause $(\mathrm{g})$ ),

$(h)_{3} \quad$ if $t_{0}<_{I} \ldots<_{I} t_{n}$ are from $\mathscr{U} \underline{\text { then }} \operatorname{tp}\left(\bar{d}_{t_{1}}^{\omega}, \bar{d}_{t_{0}}^{\omega}+\bar{c}_{t_{1}}^{\omega}\right) \vdash \operatorname{tp}\left(\bar{d}_{t_{1}}^{\omega}, M_{t_{0}}+\right.$ $\bar{d}_{t_{0}}^{\omega}+\bar{c}_{t_{0}}^{\omega}+\bar{c}_{t_{1}}^{\omega}+\ldots+\bar{c}_{t_{n}}^{\omega}$ ) (actually this is the case $m=0$ in $\left.(h)_{1}\right)$.

Remark 3.9. We easily can add:

(i) $\quad \mathbf{x}$ is normal, i.e. $\operatorname{Rang}\left(\bar{c}_{\omega}\right) \subseteq \operatorname{Rang}\left(\bar{d}_{\omega}\right)$.

Proof. First by induction on $n$ we choose $\bar{d}_{n}, \bar{c}_{n}, B_{n},\left\langle\left(\bar{c}_{t}^{n}, \bar{d}_{t}^{n}\right): t \in \mathscr{U}_{n}\right\rangle$ and if $n>0$ also $\bar{e}_{n}, \bar{e}_{t}^{n}$ (for $t \in \mathscr{U}_{n}$ ) such that:

$\circledast_{n}(a) \quad \bar{d}_{n} \in \gamma(*)+\theta \cdot n \mathfrak{C}$ and $\bar{d}_{0}=\bar{d}$ and $\bar{e}_{n} \in{ }^{\theta} \mathfrak{C}$

(b) $\mathbf{x}_{n}=\left(M_{\lambda}, B_{n}, \bar{c}_{n}, \bar{d}_{n}\right) \in \mathrm{mxK}_{\kappa, \theta}$ is normal,

(c) $\bar{d}_{m} \triangleleft \bar{d}_{n}$ if $m<n$,

(d) $\bar{c}_{m}=\bar{c}_{n}\left\lceil I_{\mathbf{x}_{m}}\right.$ if $m<n$,

(e) $\bar{c}_{t}^{n \wedge} \bar{d}_{t}^{n}$ is from $M_{\lambda}$ and realizes $\operatorname{tp}\left(\bar{c}_{n}{ }^{\wedge} \bar{d}_{n}, M_{t}\right)$ for $t \in \mathscr{U}_{n}$,

(f) $\quad \bar{d}_{n}=\bar{d}_{m}{ }^{\wedge} \bar{e}_{n}$ and $\ell g\left(\bar{e}_{n}\right)=\theta$ and $\mathbf{x}_{m} \leq_{1} \mathbf{x}_{n}$ if $n=m+1$,

(g) $\left\langle\bar{c}_{t}^{n \wedge} \bar{d}_{t}^{n}: t \in \mathscr{U}_{n}\right\rangle$ is fully indiscernible over $B_{n}+\bar{c}_{n}+\bar{d}_{n}$,

(h) $\mathscr{U}_{n} \in \mathscr{D}$ decrease with $n$ and is $\subseteq \mathscr{U}_{*}$ from 3.7 (1),

(i) if $s<t$ are from $\mathscr{U}_{n}$ then $M_{s}+\bar{c}_{s}^{n}+\bar{d}_{s}^{n} \subseteq M_{t}$ (follows by (g)),

(j) $\operatorname{tp}\left(\bar{d}_{m}, \bar{e}_{t}^{n}+\bar{c}_{m}\right) \vdash \operatorname{tp}\left(\bar{d}_{m}, M_{t}+\bar{c}_{m}+\bar{c}_{t}^{m}+\bar{d}_{t}^{m}\right)$ if $n=m+1, t \in \mathscr{U}_{n}$,

(k) $\left(M_{t}, B_{n}, \bar{c}_{n}, \bar{d}_{n}\right) \in \operatorname{mxK}_{\kappa_{I}(t), \theta}$ for $t \in \mathscr{U}_{n}$,

(l) if $n=m+1, k<\omega$ and $t_{0}<_{I} \ldots<_{I} t_{k}$ are from $\mathscr{U}_{n}$ then

$$
\operatorname{tp}\left(\bar{d}_{t_{1}}^{m}, \bar{c}_{t_{1}}^{m}+\bar{e}_{t_{0}}^{n}\right) \vdash \operatorname{tp}\left(\bar{d}_{t_{1}}^{m}, \sum_{\ell=0}^{k} \bar{c}_{t_{\ell}}^{m}+\bar{d}_{t_{0}}^{m}+\bar{e}_{t_{0}}^{n}+M_{t_{0}}\right) .
$$


Case 1: $n=0$.

First, let $\bar{d}_{0}=\bar{d}$. Recalling 2.7 (4) clearly $\mathbf{y}_{n}=:\left(M_{\lambda}, \emptyset,<>, \bar{d}_{0}\right) \in K_{\kappa, \theta}$.

Second, by Claim 2.12 we can find $B_{n}, \bar{c}_{n}$ such that $\mathbf{y}_{n} \leq_{1} \mathbf{x}_{n}:=\left(M_{\lambda}, B_{n}, \bar{c}_{n}, \bar{d}_{n}\right) \in$ $\operatorname{mxK} \mathrm{K}_{\kappa, \theta}$.

Third, for $t \in I$ we can choose $\bar{c}_{t}^{n \wedge} \bar{d}_{t}^{n}$ from $M_{\lambda}$ which realizes $\operatorname{tp}\left(\bar{c}_{n}{ }^{\wedge} \bar{d}_{n}, M_{t}\right)$.

Fourth, by 3.6 we choose $\mathscr{U}_{0} \in \mathscr{D}$ such that $\left\langle\bar{c}_{t}^{n \wedge} \bar{d}_{t}^{n}: t \in \mathscr{U}_{0}\right\rangle$ is a fully indiscernible sequence over $B_{0}+\bar{d}_{0}+\bar{c}_{0}$ and (by the normality of the filter $\mathscr{D}$ ) in particular $\bar{c}_{s}^{n \wedge} \bar{d}_{s}^{n} \subseteq M_{t}$ when $s<t \in \mathscr{U}_{0}$ are from $I$ and $\mathscr{U}_{0}$ is $\subseteq \mathscr{U}_{*}$ from 3.6. It is easy to check that all the demands hold, recalling $\bar{e}_{n}$ for $n=0$ is not required in $\circledast_{n}$.

Case 2: $n=m+1$.

First, by clause (k) for $m$ and Conclusion ?? for each $t \in \mathscr{U}_{m}$ recalling $M_{t}+\bar{c}_{t}^{m}+$ $\bar{d}_{t}^{m}$ is $\subseteq M_{\lambda}$ and of cardinality $<\kappa$ and $M_{\lambda}$ is $\kappa$-saturated, there is $\bar{e}_{t}^{n, *} \in{ }^{\theta}\left(M_{\lambda}\right)$ such that:

$$
(*)_{1} \operatorname{tp}\left(\bar{d}_{m}, \bar{c}_{m}+\bar{e}_{t}^{n, *}\right) \vdash \operatorname{tp}\left(\bar{d}_{m}, M_{t}+\bar{c}_{m}+\bar{c}_{t}^{m}+\bar{d}_{t}^{m}\right) .
$$

Second, by 3.6 choose $\mathscr{U}_{n}^{\prime} \subseteq \mathscr{U}_{m}$ which belongs to $\mathscr{D}$ such that $\left\langle\bar{c}_{t}^{m \wedge} \bar{d}_{t}^{m \wedge} \bar{e}_{t}^{n, *}: t \in\right.$ $\left.\mathscr{U}_{n}^{\prime}\right\rangle$ is fully indiscernible over $B_{m}+\bar{c}_{m}+\bar{d}_{m}$.

We shall now prove

$\odot_{1}$ if $t_{0}<\ldots<t_{k}$ are from $\mathscr{U}_{n}^{\prime} \underline{\text { then }} \operatorname{tp}\left(\bar{d}_{t_{1}}^{m}, \bar{c}_{t_{1}}^{m}+\bar{e}_{t_{0}}^{n, *}\right) \vdash \operatorname{tp}\left(\bar{d}_{t_{1}}^{m}, \bar{c}_{t_{1}}^{m}+\ldots+\right.$ $\left.\bar{c}_{t_{k}}^{m}+M_{t_{0}}\right)$.

Toward this, by clause (e) of $\circledast_{m}$ we have

$$
(*)_{2} \operatorname{tp}\left(\bar{c}_{t_{1}}^{m \wedge} \bar{d}_{t_{1}}^{m}, M_{t_{1}}\right)=\operatorname{tp}\left(\bar{c}_{m} \wedge \bar{d}_{m}, M_{t_{1}}\right) .
$$

By $(*)_{2}$ there is an elementary mapping $f$ mapping $\bar{c}_{m}{ }^{\wedge} \bar{d}_{m}$ to $\bar{c}_{t_{1}}^{m \wedge} \bar{d}_{t_{1}}^{m}$ which is the identity on $M_{t_{1}}$. But $M_{t_{0}}+\bar{c}_{t_{0}}^{m}+\bar{d}_{t_{0}}^{m} \subseteq M_{t_{1}}$ by $\circledast_{m}(i)$ and $\bar{e}_{t_{0}}^{n, *} \subseteq M_{t_{1}}$ by the full indiscernibility, i.e. by the choice of $\mathscr{U}_{n}^{\prime}$ above, hence by applying $f$ on $(*)_{1}$ for $t_{1}$ and monotonicity we get

$$
(*)_{3} \operatorname{tp}\left(\bar{d}_{t_{1}}^{m}, \bar{c}_{t_{1}}^{m}+\bar{e}_{t_{0}}^{n, *}\right) \vdash \operatorname{tp}\left(\bar{d}_{t_{1}}^{m}, M_{t_{0}}+\bar{c}_{t_{1}}^{m}+\bar{c}_{t_{0}}^{m}+\bar{d}_{t_{0}}^{m}\right) .
$$

Now by clause $(\mathrm{k})$ of $\circledast m$

$$
(*)_{4}\left(M_{t_{0}}, B_{m}, \bar{c}_{m}, \bar{d}_{m}\right) \in \operatorname{mxK}_{\kappa_{I}\left(t_{0}\right), \theta} .
$$

But by clause $(\mathrm{e})$ of $\circledast_{m}$

$$
(*)_{5} \operatorname{tp}\left(\bar{c}_{t_{1}}^{m} \wedge \bar{d}_{t_{1}}^{m}, M_{t_{0}}\right)=\operatorname{tp}\left(\bar{c}_{m} \wedge \bar{d}_{m}, M_{t_{0}}\right) .
$$

By $(*)_{4}+(*)_{5}$

$$
(*)_{6}\left(M_{t_{0}}, B_{m}, \bar{c}_{t_{1}}^{m}, \bar{d}_{t_{1}}^{m}\right) \in \operatorname{mxK}_{\kappa_{I}\left(t_{0}\right), \theta} .
$$

Also easily by clauses $(\mathrm{k})+(\mathrm{i})$ of $\circledast_{m}$ applied to $t=t_{2}, t_{3}, \ldots, t_{k}$ (recalling [1.4(3))

$$
(*)_{7} \operatorname{tp}\left(\bar{c}_{t_{2}}^{m}{ }^{\wedge} \ldots{ }^{\wedge} \bar{c}_{t_{k}}^{m}, M_{t_{0}}+\bar{c}_{t_{1}}^{m}+\bar{d}_{t_{1}}^{m}\right) \text { does not split over } B_{m} \text {. }
$$

By $(*)_{6}+(*)_{7}$ and the weak orthogonality claim 2.14(1) we have

$$
(*)_{8} \operatorname{tp}\left(\bar{d}_{t_{1}}^{m}, M_{t_{0}}+\bar{c}_{t_{1}}^{m}\right) \vdash \operatorname{tp}\left(\bar{d}_{t_{1}}^{m}, M_{t_{0}}+\bar{c}_{t_{1}}^{m}+\ldots+\bar{c}_{t_{k}}^{m}\right) .
$$


$\operatorname{By}(*)_{3}+(*)_{8}$

$$
(*)_{9} \operatorname{tp}\left(\bar{d}_{t_{1}}^{m}, \bar{c}_{t_{1}}^{m}+\bar{e}_{t_{0}}^{n, *}\right) \vdash \operatorname{tp}\left(d_{t_{1}}^{m}, M_{t_{0}}+\bar{c}_{t_{1}}^{m}+\ldots+\bar{c}_{t_{k}}^{m}\right)
$$

as promised in $\odot_{1}$.

Now we continue to deal with Case 2 , choose $F_{n}: \mathscr{U}_{n}^{\prime} \rightarrow \mathscr{U}_{n}^{\prime}$ such that $s \in$ $\mathscr{U}_{n}^{\prime} \Rightarrow s<_{I} F_{n}(s) \in \mathscr{U}_{n}^{\prime}$ and for $t \in \mathscr{U}_{n}^{\prime}$ we let $\bar{e}_{t}^{n}:=\bar{e}_{F_{n}(t)}^{n, *}$. Let $\bar{e}_{n} \in{ }^{\theta} \mathfrak{C}$ be such that $\bar{c}_{m}{ }^{\wedge} \bar{d}_{m}{ }^{\wedge} \bar{e}_{n}$ realizes $\operatorname{Av}\left(\left\langle\bar{c}_{t}^{m \wedge} \bar{d}_{t}^{m} \bar{e}_{t}^{n}: t \in \mathscr{U}_{n}^{\prime}\right\rangle / \mathscr{D}, M_{\lambda}\right)$. Let $\mathscr{U}_{n}^{\prime \prime} \in \mathscr{D}$ be $\subseteq \mathscr{U}_{n}^{\prime}$ and such that $s \in \mathscr{U}_{n}^{\prime \prime} \wedge t \in \mathscr{U}_{n}^{\prime \prime} \wedge s<_{I} t \Rightarrow F_{n}(s)<_{I} t$ and the sequence $\left\langle\bar{c}_{t}^{m \wedge} \bar{d}_{t}^{m \wedge} \bar{e}_{F(t)}^{n, *}: t \in \mathscr{U}_{n}^{\prime \prime}\right\rangle$ is fully indiscernible over $\bar{c}_{m}{ }^{\wedge} \bar{d}_{m}{ }^{\wedge} \bar{e}_{n}$.

Let $\bar{d}_{n}=\bar{d}_{m}{ }^{\wedge} \bar{e}_{n}$ and $\bar{d}_{t}^{n}=\bar{d}_{t}^{m}{ }^{\wedge} \bar{e}_{t}^{n}$ for $t \in \mathscr{U}_{n}^{\prime \prime}$.

Let $\mathbf{y}_{n}:=\left(M, B_{m}, \bar{c}_{m}, \bar{d}_{n}\right) \in K_{\kappa, \theta}$ so clearly $\mathbf{x}_{m} \leq_{1} \mathbf{y}_{n}$ hence by 2.12 there is $\mathbf{x}_{n}=\left(M_{\lambda}, B_{n}, \bar{c}_{n}, \bar{d}_{n}\right) \in \operatorname{mxK}_{\kappa, \theta}$ such that $\mathbf{y}_{n} \leq_{2} \mathbf{x}_{n}$ so $\bar{c}_{n}$ and $B_{n}$ are well defined 18 and $\bar{c}_{m}=\bar{c}_{n}\left\lceil I_{\mathbf{x}_{m}}\right.$. For $t \in \mathscr{U}_{n}^{\prime \prime}$, let $\bar{c}_{t}^{n}$ be a sequence from $M_{\lambda}$ such that $\bar{c}_{t}^{m}=\bar{c}_{t}^{n} \uparrow \operatorname{Dom}\left(\bar{c}_{m}\right)$ and $\operatorname{tp}\left(\bar{c}_{t}^{n \wedge} \bar{d}_{t}^{n}, M_{t}\right)=\operatorname{tp}\left(\bar{c}_{n} \wedge \bar{d}_{n}, M_{t}\right)$, this is possible as $M$ is $\kappa$-saturated, $\left|M_{t}+\bar{c}_{t}^{m}+\bar{d}_{t}^{n}\right|<\kappa,\left|\operatorname{Rang}\left(\bar{c}_{n}\right)\right|<\kappa$ and $\bar{c}_{t}^{m \wedge} \bar{d}_{t}^{n}$ realizes the type $\operatorname{tp}\left(\bar{c}_{m}{ }^{\wedge} \bar{d}_{n}, M_{t}\right)$.

Lastly, let $\mathscr{U}_{n}$ be a subset of $\mathscr{U}_{n}^{\prime \prime}$ which belongs to $\mathscr{D}$ such that:

$\odot_{2}\left\langle\bar{c}_{t}^{n \wedge} \bar{d}_{t}^{n}: t \in \mathscr{U}_{n}\right\rangle$ is fully indiscernible over $B_{n}+\bar{c}_{n}+\bar{d}_{n}$.

$\odot_{3}\left(M_{t}, B_{n}, \bar{c}_{n}, \bar{d}_{n}\right) \in \mathrm{mxK}_{\kappa, \theta}$ for every $t \in \mathscr{U}_{n}$.

[Why $\mathscr{U}_{n}$ exists? By 3.6 and 2.10 ,

It is easy to check that $\mathbf{x}_{n}, \bar{c}_{n}, \bar{d}_{n}, \bar{d}_{n},\left\langle\left(c_{t}^{n}, \bar{d}_{t}^{n}, \bar{e}_{t}^{n}\right): t \in \mathscr{U}_{n}\right\rangle$ are as required. E.g. clause (f) holds as $\ell g\left(\bar{d}_{0}\right)=\ell g(\bar{d})=\gamma(*)$ and $\ell g\left(\bar{d}_{m}\right)=\gamma(*)+\theta \cdot m$ by clause (a) of $\circledast_{m}$ and $\ell g\left(\bar{e}_{n}\right)=\theta$ by $\circledast_{n}(f)$ we can prove that $\ell g\left(\bar{d}_{n}\right)=\ell g\left(\bar{d}_{m}\right)+\theta=$ $\gamma(*)+\theta \cdot m+\theta=\gamma(*)+\theta n$, so we clearly are done. For clause(e) note $\odot_{1}$ and the choices of $F$ and $\mathscr{U}_{n}^{\prime \prime}, \mathscr{U}_{n}$.

So we have carried the induction. Second, let $\bar{c}_{\omega}=\bar{c}_{\lambda}^{\omega}=\bigcup_{n<\omega} \bar{c}_{n}, \bar{d}_{\omega}=\bar{d}_{t}^{\omega}=$ $\bigcup_{n<\omega} \bar{d}_{t}^{n}$ and $B=\cup\left\{B_{n}: n<\omega\right\}$ and $\mathscr{U}=\cap\left\{\mathscr{U}_{n}: n<\omega\right\}$.

Let us check that $\otimes$ from the theorem holds indeed.

Clause (a): $\mathscr{U} \in \mathscr{D}$ as each $\mathscr{U}_{n} \in \mathscr{D}$ by $\circledast_{n}(h)$ and $\mathscr{D}$ is $\kappa$-complete and $\kappa>\aleph_{0}$ recalling $\mathscr{U}=\cap\left\{\mathscr{U}_{n}: n<\omega\right\}$.

Clause (b): $B \in\left[M_{\lambda}\right]^{<\kappa}$ as $B_{n} \subseteq M_{\lambda},\left|B_{n}\right|<\kappa$ by $\circledast_{n}(b)$ for $n<\omega$ and $\kappa$ is regular uncountable recalling and $B:=\cup\left\{B_{n}: n<\omega\right\}$.

Clause $(\mathrm{c}):$ By $\circledast_{n}(a)+(c)$ for $n<\omega$.

Clause (d): $\bar{d}=\bar{d}_{0}=\bar{d}_{\omega}\lceil\gamma(*)$ is proved as in clause (c).

Clause (e): As $\mathbf{x}_{n}=\left(M, B_{n}, \bar{c}_{n}, \bar{d}_{n}\right) \in \operatorname{mxK}_{\kappa, \theta}$ by clause (b) of $\circledast_{n}$ and $\mathbf{x}_{n} \leq_{1} \mathbf{x}_{n+1}$ $\overline{\text { by } \circledast_{n+1}(f)}$ and $\left(M, B, \bar{c}_{\omega}, \bar{d}_{\omega}\right)=\cup\left\{\mathbf{x}_{n}: n<\omega\right\}$, clearly by claim 2.14 $(2)$ we are done.

Clause (f): By clause (e) of $\circledast_{n}$ (and the choice of $\bar{c}_{\omega}, \bar{d}_{\omega}, \bar{c}_{t}^{\omega}, \bar{d}_{t}^{\omega}$, etc).

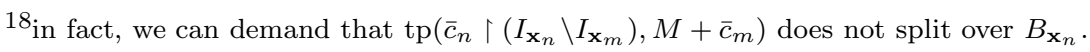


Clause $(\mathrm{g})$ : Similarly by clause $(\mathrm{g})$ of $\circledast_{n}$.

Clause $(\mathrm{h})_{1}$ : By clauses $(h)_{2}+(h)_{3}$ proved below.

Clause $(\mathrm{h})_{2}$ : By clause (i) of $\circledast_{n}$.

Clause $(\mathrm{h})_{3}$ : Holds by clause $(\ell)$ of $\circledast_{n}$.

Theorem 3.10. 1) If $M$ is $\kappa$-saturated of cardinality $\leq \lambda, \bar{d} \in \theta^{\theta^{+}}>\mathfrak{C} \underline{\text { then }}$ we can find a strict $(\kappa, \theta)$-decomposition $\left\langle\left(\bar{c}_{n}, \bar{d}_{n}\right): n<\omega\right\rangle$ over $M$ such that $\bar{d} \triangleleft \bar{d}_{0}$.

2) Instead Hypothesis [3.1 it is enough to demand: if $M$ is $\kappa$-saturated and $p=$ $p\left(\left\langle x_{i}: i<\theta\right\rangle\right)$ is a type with parameters from $M \cup C,|C| \leq \theta$ which is $(<\kappa)$ satisfiable in $M$, i.e. every subset of $p$ of cardinality $<\kappa$ is realized in $M$ then $p$ can be extended to $p^{+} \in \mathbf{S}^{\theta}(M \cup C)$ which is $(<\kappa)$-satisfiable in $M$.

Proof. 1) We can choose $\bar{M}^{\prime}=\left\langle M_{t}^{\prime}: t \in I\right\rangle$ such that $\left(M, \bar{M}^{\prime}\right)$ satisfies the demands on $\left(M_{\lambda},\left\langle M_{t}: t \in I\right\rangle\right)$ in Hypothesis 3.1(1) or 3.1(2) and apply Theorem 3.8 (as assuming $\ell g(\bar{d})=\theta$ or $\ell g(\theta)<\theta^{+}$does not matter).

2) The idea is to repeat the proof of 3.8 , but as of unclear value we leave it to the reader.

Corollary 3.11. Assume $\kappa=\lambda>|T|$ is weakly compact, $M_{\alpha} \in \mathrm{EC}_{<\lambda}(T)$ is $\prec-$ increasing continuous, $M=\cup\left\{M_{\alpha}: \alpha<\lambda\right\}$ is saturated. Then 3.8 and 3.12(2) hold.

Proof. Revise the proof of 3.8 , but in $\circledast_{n}$ weaken clauses $(\mathrm{g}),(\mathrm{h})$ to $(\mathrm{g})^{-},(\mathrm{h})^{-}$and use the proof of 4.13 in the end where

$(g)^{-} \mathscr{U}_{n} \in[\kappa]^{\kappa}$ decreasing with $n$ and $\left\langle\bar{c}_{t}^{n \wedge} \bar{d}_{t}^{n}: t \in \mathscr{U}_{n}\right\rangle$ is just fully $n$-indiscernible

$(h)^{-} \mathscr{U}_{n}$ does not belong to the weakly compact ideal.

We leave the details to the reader.

Claim 3.12. 1) (even not assuming 3.1)

Assume $M$ is $\kappa$-saturated, $|T| \leq \theta<\kappa, \operatorname{tp}\left(\bar{c}_{n}, M+\bar{c}_{0}+\ldots+\bar{c}_{n-1}\right)$ does not split over $B$ where $|B|<\kappa, B \subseteq M$ and $\ell g\left(\bar{c}_{n}\right)<\theta^{+}$and $\bar{d}_{n}=\bar{c}_{n}\lceil u$ for $n<\omega$ (so $\left.u \subseteq \operatorname{Dom}\left(\bar{c}_{n}\right)\right)$ then $\left\langle\left(\bar{c}_{n}, \bar{d}_{n}\right): n<\omega\right\rangle$ is a strict $(\kappa, \theta)$-decomposition over $(M, B)$. 2) (Assuming [3.1!') Assume $\left\langle\left(\bar{c}_{n}, \bar{d}_{n}\right): n<\omega\right\rangle$ is a strict $(\kappa, \theta)$-decomposition over $\left(M_{\lambda}, B\right)$. For any $\bar{d} \in \theta^{+}>\mathfrak{C}$ we can find a strict $(\kappa, \theta)$-decomposition $\left\langle\left(\bar{c}_{n}^{+}, \bar{d}_{n}^{+}\right)\right.$: $n<\omega\rangle$ over $\left(M_{\lambda}, B\right)$ such that $\bar{c}_{n} \unlhd \bar{c}_{n}^{+}, \bar{d}_{n} \unlhd \bar{d}_{n}^{+}$for $n<\omega$ and $\bar{d}_{0} \wedge \bar{d} \unlhd \bar{d}_{0}^{+}$.

Proof. 1) Easy.

2) Repeat the proof of 3.8 starting with $\mathbf{x}_{0}=\left(M_{\lambda}, B, \bar{c}_{0}, \bar{d}_{0}\right)$ and $\left\langle\bar{c}_{t} \bar{d}_{t}: t \in I\right\rangle$ such that $\bar{c}_{t} \wedge d_{t} \in \ell^{\ell g\left(\bar{c}_{t}\right)+\ell g\left(\bar{d}_{t}\right)} M$ realizing $\left.\operatorname{tp}\left(\bar{c}_{0} \wedge \bar{d}_{0}, M_{t}+\sum_{n<\omega} \bar{c}_{1+n} \hat{d}_{1+n}\right) . \quad \square\right]$

Claim 3.13. The sequence $\left\langle\left(\bar{c}_{t}^{2}, \bar{d}_{t}^{2}\right): t \in I_{2}\right\rangle$ is a strict $t_{0}(0, \theta)$-decomposition over $\left(B_{2}, B_{1}\right)$ when:

(a) $\left.\left\langle\left(\bar{c}_{s}^{1}, \bar{d}_{s}^{1}\right): s \in I_{1}\right)\right\rangle$ is a strict $t_{0}(0, \theta)$-decomposition $\left(\operatorname{and} \ell g\left(\bar{c}_{s}^{1}\right)=\ell g\left(\bar{c}_{t}^{2}\right), \ell g\left(\bar{d}_{s}^{1}\right)=\right.$ $\ell g\left(\bar{d}_{s}^{1}\right)=\ell g\left(\bar{d}_{t}^{2}\right)$, of course $)$, 
(b) for any $n$ if $I_{\ell} \models$ " $t_{\ell, 0}<\ldots<t_{\ell, n-1}$ " for $\ell=1,2$ then $\operatorname{tp}\left(\bar{c}_{t_{1,0}}^{1} \wedge \bar{d}_{t_{1,0}}^{1}\right.$. . . $\left.\bar{c}_{t_{1, n-1}}^{1} \wedge \bar{d}_{t_{1, n-1}}, B_{2}\right)=\operatorname{tp}\left(\bar{c}_{t_{2,0}}^{2} \wedge \bar{d}_{t_{2,0}}^{2} \wedge \cdots \bar{c}_{t_{2, n-1}}^{2} \wedge \bar{d}_{t_{2, n-1}}^{2}, B_{2}\right)$.

Proof. Should be clear. 


\section{$\S 4$. Consequences of Strict Decomposition}

Here we look again at the generic pair conjecture ([Sh:877, 0.2]). The nonstructure side (in a strong version) is proved there for $\lambda=\lambda^{<\lambda}$ non-strong limit and in [Sh:906] for $\lambda=\lambda^{<\lambda}$ strong limit (i.e. strongly inaccessible).

The conjecture is (the instances of G.C.H. are used to make the conjecture transparent):

Conjecture 4.1. The generic pair conjecture

Assume $\lambda=\overline{\lambda^{<\lambda}>|T|, 2^{\lambda}=\lambda^{+}, M_{\alpha} \in} \operatorname{EC}_{\lambda}(T)$ is $\prec$-increasing continuous for $\alpha<\lambda^{+}$with $\cup\left\{M_{\alpha}: \alpha<\lambda^{+}\right\} \in \mathrm{EC}_{\lambda^{+}}(T)$ being saturated.

1) The $\lambda$-generic pair conjecture says that: $T$ is dependent iff for some club $E$ of $\lambda^{+}$for all pairs $\alpha<\beta<\lambda^{+}$from $E$ of cofinality $\lambda^{+},\left(M_{\beta}, M_{\alpha}\right)$ has the same isomorphism type.

2) For $\varepsilon<\lambda$ the $\lambda$-generic $\zeta$-tuple conjecture says that: $T$ is dependent $\underline{\text { iff for some }}$ club $E$ of $\lambda^{+}$for all increasing sequences $\left\langle\alpha_{\varepsilon}: \varepsilon \leq \zeta\right\rangle$ of members of $E$ of cofinality $\lambda$, the structure $\left(M_{\alpha_{\zeta}}, M_{\alpha_{\varepsilon}}\right)_{\varepsilon<\zeta}$ has the same isomorphism type (equivalently, if $\left\langle\alpha_{\ell, \varepsilon}: \varepsilon \leq \zeta\right\rangle$ is as above for $\ell=1,2$ then there is an isomorphism from $M_{\alpha_{1, \zeta}}$ onto $M_{\alpha_{2, \zeta}}$ mapping $M_{\alpha_{1, \varepsilon}}$ onto $M_{2, \alpha_{\varepsilon}}$ for $\left.\varepsilon<\zeta\right)$.

We concentrate on the pair. Note that if $\kappa=\operatorname{cf}(\kappa)<\lambda$, then the $\lambda$-generic $\kappa$ tuple conjecture implies that for dependent $T$ there is a medium $(\lambda, \kappa)$-limit model, see [Sh:877], but we do not succeed to deal with it here.

Here we prove the "structure" side when $\lambda$ is measurable. It seemed natural to assume that the first order theories of such pair is complicated if $T$ is independent and "understandable" for dependent of $T$.

In fact, it may be better to ask

Problem 4.2. 1) Assume $|T|<\theta \leq \kappa \leq \lambda=\lambda^{<\kappa}<\kappa_{2} \leq \mu=\mu^{<\aleph_{2}}$ and $M_{1} \prec M_{2} \prec \mathfrak{C}, M_{1}$ is $\kappa_{1}$-saturated of cardinality $\lambda, M_{2}$ is $\kappa_{2}$-saturated of cardinality $\mu$. What can we say on $\operatorname{Th}\left(M_{2}, M_{1}\right)$ ? $\operatorname{On~}_{\mathrm{Th}_{\mathbb{L}_{\infty, \theta}(\tau(T))}}\left(M_{2}, M_{1}\right)$ ?

More generally

Problem 4.3. 1) Assume $n<\omega,|T|<\theta, \sigma<\theta<\kappa_{0}, \lambda_{\ell}=\lambda_{\ell}^{<\kappa_{\ell}}$ for $\ell \leq n, \lambda_{\ell}<$ $\kappa_{\ell+1}$ for $\ell<n$. Let $M_{\ell}$ be $\kappa_{\ell}$-saturated of cardinality $\lambda_{\ell}$ for $\ell \leq n$ and $M_{\ell} \prec M_{\ell+1}$ for $\ell<n$. What can we say on $M^{+}=\operatorname{Th}\left(M_{n}, \ldots, M_{1}, M_{0}\right)$,i.e. $M_{n}$ expanded by unary predicates for $M_{\ell}$ for $\ell<n$ ? When can we interpret (with first order formulas with parameters) second order logic on $\theta$ ? i.e. classify $T$ by this.

2) Similarly for $\mathbb{L}_{\sigma, \sigma}\left(\tau_{M^{+}}\right)$.

3) Similarly allowing $n$ to be $<\theta$.

The proof here, if e.g. $\kappa=\lambda$ is measurable say that even the $\mathbb{L}_{\infty, \kappa^{-}}$theory of the pair is constant, but does not say much even on the first order theory, (see KpSh:946). It is known that for many "complicated enough" theories $T$, for $M_{2}, M_{1}$ as in 4.2, in $\operatorname{Th}\left(M_{2}, M_{1}\right)$ we can interpret second order logic on $(\lambda,=)$. This holds, e.g. for $T=$ Peano arithmetic.

On $n$-independent theories see [Sh:886, §2]. Note that

\footnotetext{
$19_{\text {the }}$ " $2^{\lambda}=\lambda^{+}$" is just for making the formulation more transparent, and by absoluteness is equivalent to the formulation not assuming $2^{\lambda}=\lambda^{+}$.
} 
Claim 4.4. Assume $T$ is the model completion of $T_{0}$, defined below so seems "the simplest" 2-independent theory. If $M_{0} \in \mathrm{EC}_{\lambda}(T)$ and $M_{1}$ is a $\lambda^{+}$-saturated $\prec-$ extension of $M_{0} \underline{\text { then }}$ in $\left(M_{1}, M_{0}\right)$ we can interpret second order logic on $M_{0}$ (i.e. quantification on two-place relations, when:

(*) $\tau_{T_{0}}$ consists of $P_{0}, P_{1}, P_{2}$ (unary predicates) and $R$ (a ternary predicate) and a $\tau_{T_{0}}$-model $M$ is a model of $T_{0}$ iff $\left\langle P_{0}^{M}, P_{1}^{M}, P_{2}^{M}\right\rangle$ is a partition of $|M|$ and $R^{M} \subseteq P_{0}^{M} \times P_{1}^{M} \times P_{2}^{M}$.

Proof. Obvious.

Also for $T=$ theory of Boolean algebras (which is $n$-independent for every $n$ ) the theory is complicated. Of course, it would be better to eliminate the measurable assumption.

Explanation 4.5. Why $K_{\lambda, \kappa, \theta}$ and $m x K_{\lambda, \kappa, \theta}$ from $\S 2$ does not suffice for us so that in $\S 3$ we deal also with the more complicated $\left\langle\left(\bar{c}_{t}, \bar{d}_{t}\right): t \in J\right\rangle$ from 3.5? This is motivated by the proof of the generic pair conjecture.

To understand it maybe better consider the class

$(*) \mathbf{N}_{\kappa}^{2}=\left\{(N, M): M \prec N \prec \mathfrak{C}_{T}, M\right.$ is $\kappa$-saturated and $N$ is $\|M\|^{+}$saturated $\}$.

Proving the generic pair conjecture for $\kappa$ we consider $\bar{M}=\left\langle M_{\alpha}: \alpha<\kappa^{+}\right\rangle$, which is $\prec$-increasing continuous, $M=\bigcup_{\alpha} M_{\alpha} \prec \mathfrak{C}_{T}$ is saturated of cardinality $\kappa^{+}$.

Assuming $T$ is dependent we should choose a thin enough club $E$ of $\kappa^{+}$such that $\left\{\left(M_{\beta}, M_{\alpha}\right): \alpha<\beta\right.$ and $\left.\{\alpha, \beta\} \subseteq E \cap S_{\kappa}^{\kappa^{+}}\right\}$. Now the club $E$ will be chosen such that all relevant pairs $\left(M_{\beta}, M_{\alpha}\right)$ are similar enough to those of pairs from $\mathbf{N}_{\kappa}^{2}$.

So a sufficient condition for the conjecture is:

$\boxplus$ assume $\left(N_{1}, M_{1}\right),\left(N_{2}, M_{2}\right) \in \mathbf{N}_{\kappa}^{2}$, then we can find $\bar{f}=\left\langle f_{s}: s \in \mathscr{Y}\right\rangle$ such that:

(a) $\mathscr{Y}$ is a partial order,

(b) $\mathscr{Y}$ is $(<\kappa)$-complete, that is, any increasing chain (for $<\mathscr{Y}$ ) of length $<\kappa$ has an upper bound,

(c) $f_{s}$ is an $\left(N_{1}, N_{2}\right)$-elementary mapping,

(d) $\operatorname{Dom}\left(f_{s}\right)$ has cardinality $<\kappa$,

(e) $f_{s}$ maps $\operatorname{Dom}\left(f_{s}\right) \cap M_{1}$ onto $\operatorname{Rang}\left(f_{s}\right) \cap M_{2}$,

$(f)_{1}$ if $s \in \mathscr{Y}, A \in\left[N_{1}\right]^{<\kappa} \underline{\text { then }}$ for some $t \in \mathscr{Y}$ we have $s<_{Y} t \wedge A \subseteq$ $\operatorname{Dom}\left(f_{t}\right)$,

$(f)_{2}$ if $s \in \mathscr{Y}$ and $A \in\left[N_{2}\right]^{<\kappa} \underline{\text { then }}$ for some $t \in \mathscr{Y}$ we have $s<\mathscr{Y} t \wedge A \subseteq$ $\operatorname{Rang}\left(f_{t}\right)$.

Now the approximation consists of $B_{\ell} \in\left[M_{\ell}\right]^{<\kappa}$ and $\left\langle\left(\bar{c}_{n}^{\ell}, \bar{d}_{n}^{\ell}\right): n<\omega\right\rangle$, with $\bar{c}_{0}^{\ell}, \bar{d}_{0}^{\ell}$ from $N_{\ell}$, which form a strict $(\kappa,<\kappa)$-decomposition over $\left(M_{\ell}, B_{\ell}\right)$ for $\ell=1,2$ and $f$ an elementary mapping $h$ mapping $B_{1}$ onto $B_{2}$ and $\left(\bar{c}_{n}^{1}, \bar{d}_{n}^{1}\right)$ to $\left(\bar{c}_{n}^{2}, \bar{d}_{n}^{2}\right)$. So using $\bar{c}_{n}^{\ell}, \bar{d}_{n}^{\ell}$ for $n>0$ is to give us a condition with which we can continue in a good induction hypothesis.

Now it should be clear what we like to have from strict decomposition; however, the decomposition from $\S 2$ are not enough. 
We first connect decomposition (i.e. the results of 3.8) and sufficient conditions for being an indiscernible sequence.

Claim 4.6. The sequence $\left\langle\bar{a}_{\alpha}: \alpha<\alpha^{*}\right\rangle$ is an indiscernible sequence over $B \underline{\text { when }}$ for some $p, B$ we have:

(a) $\bar{a}_{\alpha}$ is a sequence of members of $\mathfrak{C}$ and $\bar{a}_{\alpha}=\bar{c}_{\alpha} \hat{d}_{\alpha}$ where $\lg \left(\bar{c}_{\alpha}\right)=\lg \left(\bar{c}_{0}\right), \ell g\left(\bar{d}_{\alpha}\right)=$ $\ell g\left(\bar{d}_{0}\right)$ (not necessarily finite),

(b) $\operatorname{tp}\left(\bar{a}_{\alpha}{ }^{\wedge} \bar{a}_{\beta}, B\right)=p$ for $\alpha<\beta<\alpha^{*}$,

(c) if $\alpha<\beta<\alpha^{*}$ then $\operatorname{tp}\left(\bar{d}_{\beta}, \bar{c}_{\beta}+\bar{d}_{\alpha}+B\right) \vdash \operatorname{tp}\left(\bar{d}_{\beta}, \bar{c}_{\beta} \cup\left\{\bar{a}_{\gamma}: \gamma \leq \alpha\right\} \cup B\right)$,

(d) $\operatorname{tp}\left(\bar{c}_{\alpha}, \cup\left\{\bar{a}_{\beta}: \beta<\alpha\right\} \cup B\right)$ is increasing with $\alpha$,

(e) $\operatorname{tp}\left(\bar{c}_{\alpha}, \cup\left\{\bar{a}_{\beta}: \beta<\alpha\right\} \cup B\right)$ does not split over $B$.

Proof. For $u, v \subseteq \alpha^{*}$ let $B_{u, v}=\bigcup\left\{\bar{a}_{\alpha}: \alpha \in u\right\} \cup \bigcup\left\{\bar{c}_{\alpha}: \alpha \in v\right\} \cup B$. For $u, v \subseteq \alpha^{*}$ and increasing functions $h_{1}$ from $u$ to $\alpha^{*}$ and $h_{2}$ to $v$ to $\alpha_{*}$ such that $h_{1} \uparrow(u \cap v)=h_{2} \uparrow(u \cap v)$ (so $h=h_{1} \cup h_{2}$ is an increasing function $h$ from $u \cup v$ to $\left.\alpha^{*}\right)$ let $f=f_{h_{1}, h_{2}}$ be defined as follows:

$\circledast(a) \quad \operatorname{Dom}(f)=B_{u, v}$,

(b) $f \nmid B=\operatorname{id}_{B}$,

(c) $f$ maps $\bar{a}_{\alpha}$ to $\bar{a}_{h(\alpha)}$ for $\alpha \in u$,

(d) $f$ maps $\bar{c}_{\alpha}$ to $\bar{c}_{h(\alpha)}$ for $\alpha \in v$.

Is $f_{h \uparrow u, h \uparrow v}$ a well defined function and even one to one? For this it suffices to check the following three demands, which follows by Clause (b) of the assumption

$(*)_{1}(\alpha) \quad$ if $\alpha, \beta<\alpha^{*}, b \in B$ and $i<\ell g\left(\bar{a}_{\alpha}\right) \underline{\text { then }}\left(\bar{a}_{\alpha}\right)_{i}=b \Leftrightarrow\left(\bar{a}_{\beta}\right)_{i}=b$,

$(\beta)$ if $\alpha, \beta<\alpha^{*}$ and $i, j<\lg \left(\bar{a}_{\alpha}\right)$ then $\left(\bar{a}_{\alpha}\right)_{i}=\left(\bar{a}_{\alpha}\right)_{j} \Leftrightarrow\left(\bar{a}_{\beta}\right)_{i}=\left(\bar{a}_{\beta}\right)_{j}$,

$(\gamma) \quad$ if $\alpha_{1}<\alpha_{2}<\alpha^{*}$ and $\beta_{1}<\beta_{2}<\alpha^{*}$ and $i, j<\ell g\left(\bar{a}_{\alpha}\right)$ then

$\left(\bar{a}_{\alpha_{1}}\right)_{i}=\left(\bar{a}_{\alpha_{2}}\right)_{j} \Leftrightarrow\left(\bar{a}_{\beta_{1}}\right)_{i}=\left(\bar{a}_{\beta_{2}}\right)_{j}$.

We prove by induction on $n$ that $\left\langle\bar{a}_{\alpha}: \alpha<\alpha^{*}\right\rangle$ is an $n$-indiscernible sequence over $B$ (when $n<\alpha^{*}$ ). For $n \leq 2$ this is trivial by Clause (b) of the assumption. So assume $n=m+1>2$ and we have proved it up to $m$. So let $\alpha_{0}<\ldots<\alpha_{m}<$ $\alpha^{*}, \beta_{0}<\ldots<\beta_{m}<\alpha^{*}$ and we shall prove that $\bar{a}_{\alpha_{0}}{ }^{\wedge} \ldots{ }^{\wedge} \bar{a}_{\alpha_{m}}, \bar{a}_{\beta_{0}}{ }^{\wedge} \ldots{ }^{\wedge} \bar{a}_{\beta_{m}}$ realize the same type over $B$, this suffices.

Now by symmetry without loss of generality $\alpha_{m} \leq \beta_{m}$ let $h_{0}=\left\{\left(\alpha_{\ell}, \beta_{\ell}\right): \ell<\right.$ $m\}, h_{1}=\left\{\left(\alpha_{\ell}, \alpha_{\ell}\right): \ell<m\right\}, h_{2}=h_{1} \cup\left\{\left(\alpha_{m}, \beta_{m}\right)\right\}$ and $h_{3}=h_{0} \cup\left\{\left(\beta_{m}, \beta_{m}\right)\right\}$ and $h_{4}=h_{0} \cup\left\{\left(\alpha_{m}, \beta_{m}\right)\right\}$.

Let $f_{0}$ be the mapping $f_{h_{0}, h_{0}}$. By the induction hypothesis

$(*)_{2} f_{0}$ is an elementary mapping.

Let $f_{1}$ be the mapping $f_{h_{1}, h_{2}}$, now by Clause (d) of the assumption

$(*)_{3} f_{1}$ is an elementary mapping.

By $(*)_{2}$ we know that $\bar{a}_{\alpha_{0}}{ }^{\wedge} \ldots{ }^{\wedge} \bar{a}_{\alpha_{m-1}}$ and $f_{0}\left(\bar{a}_{\alpha_{0}}{ }^{\wedge} \ldots{ }^{\wedge} \bar{a}_{\alpha_{m-1}}\right)$ realize the same type over $B$ and they are included in $B_{\alpha_{m}, \alpha_{m}}, B_{\beta_{m}, \beta_{m}}$ respectively. But $\alpha_{m} \leq \beta_{m}$ so both sequences are from $B_{\beta_{m}}$ hence by Clause (e) of the assumption, i.e. as $\operatorname{tp}\left(\bar{c}_{\beta_{m}}, B_{\beta_{m}, \beta_{m}}\right)$ does not split over $B$, recalling $(*)_{2}$ we have

$(*)_{4} \quad f_{2}:=f_{h_{0}, h_{3}}$ is an elementary mapping. 
By $(*)_{3}+(*)_{4}$, comparing we have

$(*)_{5} f_{3}:=f_{h_{0}, h_{4}}$ being $f_{2} \circ f_{1}$ is an elementary mapping.

Note that

$(*)_{6} f_{3}\left(\bar{c}_{\alpha_{m}}{ }^{\wedge} \bar{d}_{\alpha_{m-1}}\right)=\bar{c}_{\beta_{m}}{ }^{\wedge} \bar{d}_{\beta_{m-1}}$.

By Clause (b) of the assumption and $(*)_{5}+(*)_{6}$ clearly

$(*)_{7} f_{3}\left(\operatorname{tp}\left(\bar{d}_{\alpha_{m}}, \bar{c}_{\alpha_{m}}+\bar{d}_{\alpha_{m-1}}+B\right)\right)=\operatorname{tp}\left(\bar{d}_{\beta_{m}}, \bar{c}_{\beta_{m}}+\bar{d}_{\beta_{m-1}}+B\right)$.

By Clause (c) of the assumption

$(*)_{8} \operatorname{tp}\left(\bar{d}_{\alpha_{m}}, \bar{c}_{\alpha_{m}}+\bar{d}_{\alpha_{m-1}}+B\right) \vdash \operatorname{tp}\left(\bar{d}_{\alpha_{m}}, \bar{c}_{\alpha_{m}}+\bar{a}_{\alpha_{0}}+\ldots+\bar{a}_{\alpha_{m-1}}+B\right)$

$(*)_{9} \operatorname{tp}\left(\bar{d}_{\beta_{m}}, \bar{c}_{\beta_{m}}+\bar{d}_{\beta_{m-1}}+B\right) \vdash \operatorname{tp}\left(\bar{d}_{\beta_{m}}, \bar{c}_{\beta_{m}}+\bar{a}_{\beta_{0}}+\ldots+\bar{a}_{\beta_{m-1}}+B\right)$.

Together $f_{4}=f_{h_{4}, h_{4}}$ is an elementary mapping and it maps $\bar{a}_{\alpha_{\ell}}$ to $\bar{a}_{\beta_{\ell}}$ for $\ell \leq m$ (and extend $\operatorname{id}_{B}$ ) so we are done.

Observation 4.7. The sequence $\left\langle\left(\bar{c}_{t}, \bar{d}_{t}\right): t \in I\right\rangle$ is a strict $_{0}(0, \theta)$-decomposition over $\left(B^{0}, B\right)$ when for some $\left\langle I_{\ell}, B, B^{\ell}: \ell<n\right\rangle$ we have:

(a) $n<\omega$ and $n \geq 2$

(b) the linear order $I$ is $I_{0}+\ldots+I_{n}$ where $I_{\ell}$ is infinite for $\ell=1, \ldots, n-1$,

(c) $\left\langle\left(\bar{c}_{t}, \bar{d}_{t}\right): t \in I_{\ell}\right\rangle$ is a $\operatorname{strict}_{0}(0, \theta)$-decomposition over $\left(B^{\ell}, B\right)$ for $\ell \leq n$,

(d) $\left\langle\bar{c}_{t}{ }^{\wedge} \bar{d}_{t}: t \in I_{\ell}+I_{\ell+1}\right\rangle$ is indiscernible over $B^{\ell}$ and $\ell g\left(\bar{c}_{t}\right)$ for $t \in I_{\ell} \cup I_{\ell+1}$ is constant for $\ell<n$,

(e) $B^{\ell+1} \supseteq\left\{\bar{c}_{t} \wedge \bar{d}_{t}: t \in I_{\ell}\right\} \cup B^{\ell}$,

(f) $B \subseteq B^{0}$.

Proof. In Definition 3.5. Clause (a) holds trivially as $B^{0} \supseteq B$ by Clause (f) here (recalling that $\kappa$ there stands for 0 here). For Clause (b) of 3.5 the sequence $\left\langle\ell g\left(\bar{c}_{t}\right): t \in I\right\rangle$ is constant as for each $\ell<n$ as the sequence $\left\langle\ell g\left(\bar{c}_{t}\right): t \in I_{\ell} \cup I_{\ell+1}\right\rangle$ is constant (by Clause (c) here and (b) in 3.5) and use transitivity of equality and $I_{\ell}$ for $\ell=1, \ldots, n-1$ being non-empty by Clause (b) here. Similarly $\left\langle\ell g\left(\bar{d}_{t}\right): t \in I\right\rangle$ is constant, so 3.5 (b) indeed holds.

Similarly, Clause 3.5 (c) follows from 3.5(d) proved below and also Clause (c) here for $\ell=1$ (recalling $I_{1}$ is infinite). Clause $3.5(\mathrm{e})^{-}$follows similarly using $B^{\prime} \supseteq B^{0}$ by Clause (e) here and $(e)^{-}$there

So we are left with Clause $3.5(\mathrm{~d})$, that is $\left\langle\bar{c}_{t} \bar{d}_{t}: t \in I\right\rangle$ is an indiscernible sequence over $B^{0}$. For this we prove by induction on $k \leq n$ that $\left\langle\bar{c}_{t} \hat{d}_{t}: t \in\right.$ $\left.\cup\left\{I_{\ell}: \ell \in[n-k, n]\right\}\right\rangle$ is an indiscernible sequence over $B^{n-k}$. For $k=0,1$ this holds by clause (d), for $k \geq 2$, let $s_{0}<_{I} \ldots<_{I} s_{m-1}, t_{0}<_{I} \ldots<_{I} t_{m-1}$ be from $\cup\left\{I_{\ell}: \ell \in[n-k, n]\right\}$ be given. Choose $s_{i}^{\prime}, t_{i}^{\prime}$ from $I_{n-k} \cup I_{n-k+1}$ for $i<m$ such that $s_{0}^{\prime}<_{I} \ldots<_{I} s_{m-1}^{\prime}, t_{0}^{\prime}<_{I} \ldots<_{I} t_{m-1}^{\prime}$ and $s_{\ell} \in I_{n-k} \Rightarrow s_{\ell}^{\prime}=s_{\ell}, s_{\ell} \notin I_{n-k} \Rightarrow$ $s_{\ell}^{\prime} \in I_{n-k+1}$ and $t_{\ell} \in I_{n-k} \Rightarrow t_{\ell}^{\prime}=t_{\ell}, t_{\ell} \notin I_{n-k} \Rightarrow t_{\ell}^{\prime} \in I_{n-k+1}$. This is possible as $I_{n-k+1}$ is infinite because $k \in[2, n]$.

Let $j \leq m$ be such that $\ell<m \Rightarrow\left(s_{\ell} \in I_{n-k} \Leftrightarrow \ell<j\right)$; so by the induction hypothesis we have $\operatorname{tp}\left(\bar{a}_{s_{j}}{ }^{\wedge} \ldots{ }^{\wedge} \bar{a}_{s_{m-1}}, B^{n-k+1}\right)=\operatorname{tp}\left(\bar{a}_{s_{j}^{\prime}}{ }^{\wedge} \ldots{ }^{\wedge} \bar{a}_{s_{m-1}^{\prime}}, B^{n-k+1}\right)$. As $\ell<j \Rightarrow \bar{a}_{s_{\ell}}=\bar{a}_{s_{\ell}^{\prime}} \subseteq B^{n-k+1}$ and $B^{n-k} \subseteq B^{n-k+1}$ it follows that $\operatorname{tp}\left(\bar{a}_{s_{0}}{ }^{\wedge} \ldots{ }^{\wedge} \bar{a}_{s_{m-1}}, B^{n-k}\right)$ is equal to $\operatorname{tp}\left(\bar{a}_{s_{0}^{\prime}}{ }^{\wedge} \ldots{ }^{\wedge} \bar{a}_{s_{m-1}^{\prime}}, B^{n-k}\right)$. This type by clause (d) is equal to $\operatorname{tp}\left(\bar{a}_{t_{0}^{\prime}}{ }^{\wedge} \ldots{ }^{\wedge} \bar{a}_{t_{m-1}^{\prime}}, B^{n-k}\right)$. 
Similarly to the proof in the beginning of the paragraph, this type by the induction hypothesis is equal to $\operatorname{tp}\left(\bar{a}_{t_{0}}{ }^{\wedge}{ }^{\wedge} \bar{a}_{t_{m-1}}, B^{n-k}\right)$, so together we are done.

The following is a local version of 4.6 (see 4.9)

Claim 4.8. Assume $(n(*)<\omega$ and $\alpha(*)>n(*))$ for each $k<n(*))$

(a) $\bar{c}_{\alpha}^{k} \in{ }^{\gamma(k, 1)} \mathfrak{C}$ for $k \leq n(*), \alpha<\alpha(*)$; this means that the length of $\bar{c}_{\alpha}^{k}$ may depend on $m$ but not on $\alpha$ and may be infinite,

(b) $\bar{d}_{\alpha}=\bar{d}_{\alpha}^{0} \in{ }^{\gamma(k, 0)} \mathfrak{C}$ for $\alpha<\alpha(*)$,

(c) $\bar{c}_{\alpha}^{k} \triangleleft \bar{c}_{\alpha}^{k+1}$ for $\alpha<\alpha(*), k<n(*)$,

(d) $\bar{e}_{\alpha}^{k} \in \gamma(m, 2) \mathfrak{C}$ for non-zero $k \leq n(*), \alpha<\alpha(*)$,

(e) for all $\alpha<\beta<\alpha(*)$ the type $\operatorname{tp}\left(\bar{c}_{\beta}^{k+1}{ }^{\wedge} \bar{e}_{\alpha}^{k+1}{ }^{\wedge} \bar{d}_{\beta}{ }^{\wedge} \bar{e}_{\beta}^{k}, B\right)$ is the same,

(f) $\operatorname{tp}\left(\bar{d}_{\beta}{ }^{\wedge} \bar{e}_{\beta}^{k}, \bar{c}_{\beta}^{k}+\bar{e}_{\alpha}^{k+1}+B\right) \vdash \operatorname{tp}\left(\bar{d}_{\beta}^{k \wedge} \bar{e}_{\beta}^{k}, \bar{c}_{\beta}^{k}+\sum_{\gamma<\beta} \bar{c}_{\gamma}^{k \wedge} \bar{d}_{\gamma}+B\right)$,

(g) $\operatorname{tp}\left(\bar{c}_{\alpha}^{k}, \cup\left\{\bar{c}_{\beta}^{k+1 \wedge} \bar{d}_{\beta}{ }^{\wedge} \bar{e}_{\beta}^{k+1}: \beta<\alpha\right\} \cup B\right)$ is increasing with $\alpha$,

(h) $\operatorname{tp}\left(\bar{c}_{\alpha}^{k}, \cup\left\{\bar{c}_{\beta}^{k+1} \wedge \bar{d}_{\beta}{ }^{\wedge} \bar{e}_{\beta}^{k+1}: \beta<\alpha\right\} \cup B\right)$ does not split over $B$,

(i) $\operatorname{tp}\left(\bar{c}_{\alpha}^{n(*) \wedge} \bar{d}_{\alpha}{ }^{\wedge} \bar{e}_{\alpha}^{k}, B\right)$ is the same for all $\alpha<\alpha(*)$.

Then $\left\langle\bar{c}_{\alpha}^{0}{ }^{\wedge} \bar{d}_{\alpha}: \alpha<\alpha^{*}\right\rangle$ is an $n(*)$-indiscernible sequence over $B$.

Remark 4.9. 1) In what sense is 4.8 a local version of 4.6? In the second we get only $n(*)$-indiscernibility. Note that the role of $\bar{d}_{\alpha}$ there is played by $\bar{d}_{\alpha}, \bar{e}_{\alpha}^{m}(m<n(*))$ here.

2) The claim is not used in the rest of the section.

Proof. We prove by induction on $n<n(*)$ that

$\odot_{1}$ if $k \leq n(*)-n$ and $\alpha_{0}<\ldots<\alpha_{n}$ and $\beta_{0}<\beta_{1}<\ldots<\beta_{n}$ then

$\bar{c}_{\alpha_{0}}^{k} \wedge_{\alpha_{0}}{ }^{\wedge} \ldots{ }^{\wedge} \bar{c}_{\alpha_{n-1}}^{k}{ }^{\wedge} \bar{d}_{\alpha_{n-1}}{ }^{\wedge} \bar{c}_{\alpha_{n}}^{k}{ }^{\wedge} \bar{d}_{\alpha_{n}}{ }^{\wedge} \bar{e}_{\alpha_{n}}^{k}$ and

$\bar{c}_{\beta_{0}}^{k}{ }^{\wedge} \bar{d}_{\beta_{0}}{ }^{\wedge} \ldots{ }^{\wedge} \bar{c}_{\alpha_{n-1}}^{k}{ }^{\wedge} \bar{d}_{\alpha_{n-1}}{ }^{\wedge} \bar{c}_{\beta_{n}}^{k}{ }^{\wedge} \bar{d}_{\beta_{n}}{ }^{\wedge} \bar{e}_{\beta_{n}}^{k}$ realize the same type over $B$.

For $n=n(*)-1, k=0$ we get the desired conclusion.

For $n=0$ this holds by clause (i) of the assumption. So assume $n=m+1$ and $k \leq n(*)-n$ and we have proved this for $m$. Note that $k+1 \leq n(*)-m$. So let $\alpha_{0}<\ldots<\alpha_{m+1}<\alpha^{*}, \beta_{0}<\ldots<\beta_{m+1}<\alpha^{*}$ be given and without loss of generality $\alpha_{n} \leq \beta_{n}$ and we shall proof the equality of types from $\odot_{1}$ in this case, this suffice. Now

$(*)_{1} \bar{c}_{\alpha_{0}}^{k+1} \wedge \bar{d}_{\alpha_{0}} \wedge \ldots \bar{c}_{\alpha_{m-1}}^{k+1} \wedge \bar{d}_{\alpha_{m-1}}{ }^{\wedge} \bar{c}_{\alpha_{m}}^{k+1} \wedge \bar{d}_{\alpha_{m}}{ }^{\wedge} \bar{e}_{\alpha_{m}}^{k+1}$ and $\bar{c}_{\beta_{0}}^{k+1} \wedge \bar{d}_{\beta_{0}} \wedge^{\wedge}{ }^{\wedge} \bar{c}_{\beta_{m-1}}^{k+1} \wedge \bar{d}_{\alpha_{m-1}}^{k+1}{ }^{\wedge} \bar{c}_{\alpha_{m}}^{k+1} \wedge \bar{d}_{\alpha_{m}}{ }^{\wedge} e_{\alpha_{m}}^{k+1}$ realize

the same type over $B$.

[Why? By the induction hypothesis.]

$(*)_{2} \operatorname{tp}\left(\bar{c}_{\beta_{n}}^{k}, \cup\left\{\bar{c}_{\gamma}^{k+1}{ }^{\wedge} \bar{d}_{\gamma}{ }^{\wedge} \bar{e}_{\gamma}^{k+1}: \gamma<\beta_{n}\right\} \cup B\right)$ extends $\operatorname{tp}\left(\bar{c}_{\alpha_{n}}^{k}, \cup\left\{\bar{c}_{\gamma}^{k+1}{ }^{\wedge} \bar{d}_{\gamma}{ }^{\wedge} \bar{e}_{\gamma}^{k+1}:\right.\right.$ $\left.\left.\gamma<\alpha_{n}\right\} \cup B\right)$.

[Why? By clause (g) of the assumption.]

$(*)_{3} \operatorname{tp}\left(\bar{c}_{\beta_{n}}^{k}, \cup\left\{\bar{c}_{\gamma}^{k+1} \bar{d}_{\gamma}{ }^{\wedge} \bar{e}_{\gamma}^{k+1}: \gamma<\beta_{n}\right\} \cup B\right)$ does not split over $B$. 
[Why? By clause (h) of the assumption.]

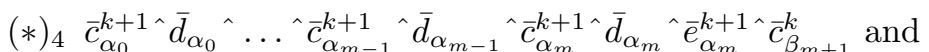

$\bar{d}_{\beta_{0}}^{k+1 \wedge} \bar{d}_{\beta_{0}}{ }^{\wedge} \ldots{ }^{\wedge} \bar{c}_{\beta_{m-1}}^{k+1} \wedge \bar{d}_{\beta_{m-1}}{ }^{\wedge} \bar{c}_{\beta_{m}}^{k+1 \wedge} \bar{d}_{\beta_{m}}{ }^{\wedge} \bar{e}_{\beta_{m}}^{k+1}{ }^{\wedge} \bar{c}_{\beta_{m+1}}^{k}$ realize the same type over $B$.

[Why? By $(*)_{1}+(*)_{3}$.]

$(*)_{5}$ in $(*)_{4}$ we can replace $\bar{c}_{\beta_{m+1}}^{k}$ in the first sequence by $\bar{c}_{\alpha_{m+1}}^{k}$.

[Why? By $(*)_{4}+(*)_{2}$.] But

$(*)_{6}\left(\bar{c}_{\alpha_{m+1}}^{k+1}{ }^{\wedge} \bar{e}_{\alpha_{m}}^{k+1}\right)^{\wedge}\left(\bar{d}_{\alpha_{m+1}}{ }^{\wedge} \bar{e}_{\alpha_{m+1}}^{k}\right)$ and $\left(\bar{c}_{\beta_{m+1}}^{k+1}{ }^{\wedge} \bar{e}_{\beta_{m}}^{k+1}\right)^{\wedge}\left(\bar{d}_{\beta_{m+1}}{ }^{\wedge} \bar{e}_{\beta_{m+1}}^{k}\right)$ realize the same type over $B$.

[Why? By clause (e) of the assumption.]

$(*)_{7}$ in $(*)_{4},(*)_{5}$ we can replace $\bar{c}_{\alpha_{\ell}}^{k+1}(\ell \leq m)$ by $\bar{c}_{\alpha_{\ell}}^{k}$.

[Why? As $\bar{c}_{\gamma}^{k} \triangleleft \bar{c}_{\gamma}^{k+1}$ by clause (c) of the assumption.]

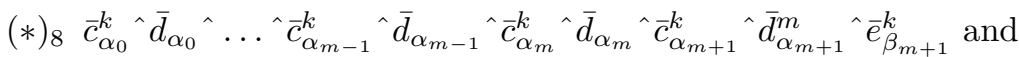
$\bar{c}_{\beta_{0}}^{k}{ }^{\wedge} \bar{d}_{\beta_{0}}{ }^{\wedge} \ldots{ }^{\wedge} \bar{c}_{\beta_{m-1}}^{k}{ }^{\wedge} \bar{d}_{\beta_{m-1}}{ }^{\wedge} \bar{c}_{\beta_{m}}^{k}{ }^{\wedge} \bar{d}_{\beta_{m}}{ }^{\wedge} \bar{c}_{\beta_{m+1}}^{k}{ }^{\wedge} \bar{d}_{\beta_{m+1}}{ }^{\wedge} \bar{e}_{\beta_{m+1}}^{k}$ realize the same type over $B$.

[Why? By $(*)_{7}+(*)_{6}$ and clause (f) of the assumption.]

We finish the induction step. Hence we get the desired statement.

We now return to the generic pair conjecture. Central here is the following definition; the best case is $\lambda=\kappa=\theta$ is a measurable cardinal.

Definition 4.10. We say that the triple $(\lambda, \kappa,<\theta)$ is good or $T$-good when:

(A) $|T|<\theta=\operatorname{cf}(\theta) \leq \kappa=\operatorname{cf}(\kappa) \leq \lambda=\lambda^{<\kappa}$,

(B) $T$ is dependent,

$(C)$ if $M$ is $\kappa$-saturated of cardinality $\leq \lambda$ and $\bar{d} \in{ }^{\theta>} \mathfrak{C}$ then we can find $B \subseteq M$ of cardinality $<\kappa$ and a strict $(\kappa,<\theta)$-decomposition $\left\langle\left(\bar{c}_{n}, \bar{d}_{n}\right): n<\omega\right\rangle$ over $(M, B)$ such that $\bar{d} \unlhd \bar{d}_{0}$,

$(D)$ if $M$ is $\kappa$-saturated of cardinality $\leq \lambda, B \subseteq M$ has cardinality $<\kappa,\left\langle\left(\bar{c}_{n}, \bar{d}_{n}\right)\right.$ : $n\langle\omega\rangle$ is a strict $(\kappa,<\theta)$-decomposition over $(M, B)$ and $\bar{d} \in{ }^{\theta>} \mathfrak{C}$ then there is a strict $(\kappa,<\theta)$-decomposition $\left\langle\left(\bar{c}_{n}^{+}, \bar{d}_{n}^{+}\right): n<\omega\right\rangle$ over $(M, B)$ such that $\bar{c}_{n} \unlhd \bar{c}_{n}^{+}, \bar{d}_{n} \triangleleft \bar{d}_{n}^{+}$for $n<\omega$ and $\bar{d}_{0} \wedge \bar{d} \unlhd \bar{d}_{0}^{+}$.

So to begin our analysis we need

Observation 4.11. 1) If $T$ is a dependent and $\lambda>|T|$ is a measurable cardinal then $(\lambda, \lambda,<\lambda)$ is $T$-good.

2) If $T$ is dependent, $\kappa$ a supercompact cardinality and $\lambda=\lambda^{<\lambda} \geq \kappa$, then $(\lambda, \kappa,<$ $\kappa)$ is $T$-good.

Proof. Immediate by 3.12(2) and 3.7(2), you may use 3.8, too. 
For the rest of this section we assume, till but not including the end that is, 4.21.

Hypothesis 4.12. 1) $T$ is dependent.

2) $|T|<\theta=\operatorname{cf}(\theta) \leq \kappa \leq \lambda$ and $\lambda=\lambda^{<\lambda}>|T|$ and $(\lambda, \kappa,<\theta)$ is $T$-good.

Claim 4.13. Assume

(a) $\delta$ is a limit ordinal $<\theta=\operatorname{cf}(\theta)$,

(b) $\left\langle\left(\bar{c}_{n}^{\alpha}, \bar{d}_{n}^{\alpha}\right): n<\omega\right\rangle$ is a strict $(\kappa,<\theta)$-decomposition over $\left(M, B_{\alpha}\right)$ for each $\alpha<\delta$,

(c) $\bar{c}_{n}^{\alpha} \unlhd \bar{c}_{n}^{\beta} \wedge \bar{d}_{n}^{\alpha} \unlhd \bar{d}_{n}^{\beta}$ for $\alpha<\beta<\delta, n<\omega$,

(d) $B_{\alpha} \subseteq B_{\beta}$ for $\alpha<\beta<\delta$,

(e) we define $\bar{c}_{n}^{\delta}=\cup\left\{\bar{c}_{n}^{\alpha}: \alpha<\delta\right\}, \bar{d}_{n}^{\delta}=\cup\left\{\bar{d}_{n}^{\alpha}: \alpha<\delta\right\}, B_{\delta}=\cup\left\{B_{\alpha}: \alpha<\delta\right\}$.

$\underline{\text { Then }}\left\langle\left(\bar{c}_{n}^{\delta}, \bar{d}_{n}^{\delta}\right): n<\omega\right\rangle$ is a strict $(\kappa,<\theta)$-decomposition over $\left(M, B_{\delta}\right)$.

Proof. We have to check Clauses (a)-(f) of Definition 3.5(1). Clause (a) is trivial by assumption (b) of 4.13 recaling $\delta<\theta=\operatorname{cf}(\theta)$. Clause (b) holds as $\delta<\theta=\operatorname{cf}(\theta)$ by assumption (a) of 4.13 and $\left\langle\ell g\left(\bar{c}_{n}^{\delta}\right): n<\omega\right\rangle$ is constant by assumptions (b),(c) and similarly $\left\langle\ell g\left(\bar{d}_{n}^{\delta}\right): n<\omega\right\rangle$ is constant. Next Clauses (c),(d),(e) hold by their local character and assumptions (b) + (c) of 4.13 .

Lastly, proving Clause (f) is the main point, it means to show:

$\odot_{1}$ if $B_{\delta} \subseteq A \subseteq M$ and $|A|<\kappa$ then for some pair $(\bar{c}, \bar{d})$ of sequences from $M$ we have $\left\langle\bar{c}^{\wedge} \bar{d}\right\rangle^{\wedge}\left\langle\bar{c}_{n}^{\delta} \bar{d}_{n}^{\delta}: n<\omega\right\rangle$ is an indiscernible sequence over $A$.

Toward this by induction on $\alpha<\delta$ we choose a pair $\left(\bar{c}_{\alpha}^{*}, \bar{d}_{\alpha}^{*}\right)$ such that

$\circledast_{1}\left\langle\bar{c}_{\alpha}^{*}{ }^{\wedge} \bar{d}_{\alpha}^{*}\right\rangle^{\wedge}\left\langle c_{n}^{\alpha \wedge} \bar{d}_{n}^{\alpha}: n<\omega\right\rangle$ is an indiscernible sequence over $A \cup \bigcup\left\{\bar{c}_{\beta}^{* \wedge} \bar{d}_{\beta}^{*}:\right.$ $\beta<\alpha\}$.

[Why possible? We can choose $\left(\bar{c}_{\alpha}^{*}, \bar{d}_{\alpha}^{*}\right)$ because $\left\langle\left(\bar{c}_{n}^{\alpha}, \bar{d}_{n}^{\alpha}\right): n<\omega\right\rangle$ being a strict $(\kappa,<\theta)$-decomposition over $B_{\alpha}$, we can apply clause (f) of Definition 3.5 recalling $B_{\alpha}$ being a subset of $A \cup\left\{\left(\bar{c}^{* \wedge} \bar{d}^{*}: \beta<\alpha\right\}\right.$ and the later being $\subseteq M$ and of cardinality $<\kappa$ as $\kappa$ is regular $\geq \theta>\delta$.]

We can find $\left\langle\left(\bar{c}_{\alpha}, \bar{d}_{\alpha}\right): \alpha<\delta\right\rangle$ though not necessarily in $M$ such that

$\circledast_{2}(a)\left\langle\bar{c}_{\alpha}{ }^{\wedge} \bar{d}_{\alpha}: \alpha<\delta\right\rangle^{\wedge}\left\langle\bar{c}_{n}^{\delta}{ }^{\wedge} \bar{d}_{n}^{\delta}: n<\omega\right\rangle$ is an indiscernible sequence over $A$

(b) $\bar{c}_{\alpha}^{*} \unlhd \bar{c}_{\alpha}$ and $\bar{d}_{\alpha}^{*} \unlhd \bar{d}_{\alpha}$ for $\alpha<\delta$ and, of course,

$$
\ell g\left(\bar{c}_{\alpha}^{*}\right)=\ell g\left(\bar{c}_{\alpha}^{\delta}\right), \ell g\left(\bar{d}_{\alpha}\right)=\ell g\left(\bar{d}_{\alpha}^{\delta}\right) .
$$

[Why? For this by using the saturation of $\mathfrak{C}$, it is enough to prove that: if $n<$ $m<\omega, \alpha_{0}<\ldots<\alpha_{n-1}<\alpha_{n}<\delta$ then the sequences $\bar{c}_{\alpha_{0}}^{*}{ }^{\wedge} \bar{d}_{\alpha_{0}}^{*}{ }^{\wedge} \ldots{ }^{\wedge} \bar{c}_{\alpha_{n-1}}^{*}{ }^{\wedge} \bar{d}_{\alpha_{n-1}}^{*}$ $\bar{c}_{0}^{\alpha_{0} \wedge} \bar{d}_{0}^{\alpha_{0} \wedge} \bar{c}_{1}^{\alpha_{1} \wedge} \bar{d}_{1}^{\alpha_{1} \wedge} \ldots{ }^{\wedge} \bar{c}_{n-1}^{\alpha_{n-1} \wedge} \bar{d}_{n-1}^{\alpha_{n-1}}$ realize the same type over $A \cup\left\{\bar{c}_{k}^{\alpha_{n} \wedge} \bar{d}_{k}^{\alpha_{n}}: k \in\right.$ $[n, m)\}$. This is proved by induction on $n<\omega$ and is straight.]

Hence we can find a pair $\left(\bar{c}^{\prime}, \bar{d}^{\prime}\right)$ such that:

$\circledast_{3}\left\langle\bar{c}^{\prime \wedge} \bar{d}^{\prime}\right\rangle^{\wedge}\left\langle\bar{c}_{\alpha}{ }^{\wedge} \bar{d}_{\alpha}: \alpha<\delta\right\rangle^{\wedge}\left\langle\bar{c}_{n}^{\delta}{ }^{\wedge} \bar{d}_{n}^{\delta}: n<\omega\right\rangle$ is an indiscernible sequence over $A$.

Lastly, we choose $\left(\bar{c}^{\prime \prime}, \bar{d}^{\prime \prime}\right)$ such that 
$\circledast_{4}\left(\bar{c}^{\prime \prime}, \bar{d}^{\prime \prime}\right)$ is a pair of sequences from $M$ such that $\bar{c}^{\prime \prime \wedge} \bar{d}^{\prime \prime}$ realizes $\operatorname{tp}\left(\bar{c}^{\prime \wedge} \bar{d}^{\prime}, A \cup\right.$ $\left.\bigcup\left\{\bar{c}_{\alpha}^{*} \wedge \bar{d}_{\alpha}^{*}: \alpha<\delta\right\}\right)$, of course with $\ell g\left(\bar{c}^{\prime \prime}\right)=\ell g\left(\bar{c}_{0}^{\delta}\right), \ell g\left(\bar{d}^{\prime \prime}\right)=\ell g\left(\bar{d}_{0}^{\delta}\right)$; equivalently there is an automorphism of $\mathfrak{C}$ which is the identity on $A \cup \bigcup\left\{\bar{c}_{\alpha}^{*} \bar{d}_{\alpha}^{*}\right.$ : $\alpha<\delta\}$ mapping $\bar{c}^{\prime \wedge} \bar{d}^{\prime}$ to $\bar{c}^{\prime \prime \wedge} \bar{d}^{\prime \prime}$.

We shall prove that $\left(\bar{c}^{\prime \prime}, \bar{d}^{\prime \prime}\right)$ is as required. Now to prove that $\left(\bar{c}^{\prime \prime}, \bar{d}^{\prime \prime}\right)$ is as required in $\odot_{1}$ it suffices to prove, for each $\alpha<\delta$ that

$$
\odot_{2}\left\langle\left(\bar{c}^{\prime \prime}\left\lceil\ell g\left(\bar{c}_{0}^{\alpha}\right)\right)^{\wedge}\left(\bar{d}^{\prime \prime}\left\lceil\ell g\left(\bar{d}_{0}^{\alpha}\right)\right)\right\rangle^{\wedge}\left\langle\bar{c}_{n}^{\alpha \wedge} \bar{d}_{n}^{\alpha}: n<\omega\right\rangle\right.\right.
$$

is an indiscernible sequence over $A$.

[Why? As $\odot_{1}$ is a "local" demand, i.e. it says that $\bar{c}^{\prime \prime \wedge} \bar{d}^{\prime \prime}$ is a sequence realizing an appropriate type $q$ (and is from $M$ ) and for this it suffices to check every finite subtype so $\odot_{2}$ suffices.]

Now $\odot_{2}$ follows by $\circledast_{6}$ below. Let $\left(\bar{c}_{\beta, \gamma}^{*}, \bar{d}_{\beta, \gamma}^{*}\right)=\left(\bar{c}_{\beta}^{*}\left\lceil\ell g\left(\bar{c}_{0}^{\gamma}\right), \bar{d}_{\beta}^{*}\left\lceil\ell g\left(d_{0}^{\gamma}\right)\right)\right.\right.$ and $\left(\bar{c}_{\gamma}^{\prime \prime}, \bar{d}_{\gamma}^{\prime \prime}\right)=\left(\bar{c}^{\prime \prime}\left|\ell g\left(\bar{c}_{0}^{\gamma}\right), \bar{d}^{\prime \prime}\right| \ell g\left(\bar{d}_{0}^{\gamma}\right)\right)$ for $\gamma<\delta$ and $\beta<\delta$; we use only $\beta \in[\gamma, \delta)$.

\section{Now}

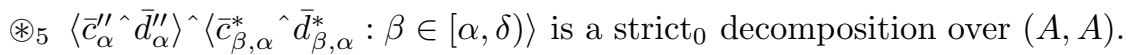

$\left[\right.$ Why? By $\circledast_{3}$, this holds for $\left\langle\left(\bar{c}^{\prime}\left\lceil\ell g\left(\bar{c}_{0}^{\alpha}\right)\right)^{\wedge}\left(\bar{d}^{\prime}\left\lceil\ell g\left(\bar{c}_{0}^{\alpha}\right)\right)\right\rangle^{\wedge}\left\langle\bar{c}_{\beta, \alpha}^{*}{ }^{\wedge} \bar{d}_{\beta, \alpha}^{*}: \beta \in[\alpha, \delta)\right\rangle\right.\right.$ and we use preservation by automorphism of $\mathfrak{C}$, i.e. use $\circledast_{4}$.]

$\circledast_{6}$ For $\alpha<\delta$ and $i \leq \omega$ the sequence $\left\langle\bar{c}_{\alpha}^{\prime \prime \wedge} \bar{d}_{\alpha}^{\prime \prime}\right\rangle^{\wedge}\left\langle\bar{c}_{\beta, \alpha}^{*}{ }^{\wedge} \bar{d}_{\beta, \alpha}^{*}: \beta \in[\alpha, \delta)\right\rangle^{\wedge}\left\langle c_{n}^{\alpha \wedge} \bar{d}_{n}^{\alpha}:\right.$

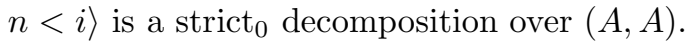

[Why? We prove this by induction on $i$, noticing that for $i=\omega$ we get the desired conclusion; also for $i=\omega$ the inductive step is trivial and for $i=0$ use $\circledast_{5}$. So assume $i=n+1$, let

$(*)_{1} \bullet A_{1}=A \cup \operatorname{Rang}\left(\bar{c}_{\alpha}^{\prime \prime}\right) \cup \operatorname{Rang}\left(\bar{d}_{\alpha}^{\prime \prime}\right)$,

- $A_{2}=A_{2, \delta}$ where

- for $\gamma \in[\alpha, \delta]$ we let $A_{2, \gamma}=\cup\left\{\operatorname{Rang}\left(\bar{c}_{\beta, \alpha}^{*}\right) \cup \operatorname{Rang}\left(\bar{d}_{\beta, \alpha}^{*}\right): \beta \in[\alpha, \gamma)\right\}$,

- $A_{3}=\cup\left\{\bar{c}_{\ell}^{\alpha \wedge} \bar{d}_{\ell}^{\alpha}: \ell<n\right\}$.

Clearly

$(*)_{2} \quad(a) \quad A_{1}, A_{2, \delta}$ are $\subseteq M$ and $A_{3} \subseteq \mathfrak{C}$,

(b) $A_{2, \gamma}$ is $\subseteq$-increasing for $\gamma \in[\alpha, \delta)$.

Let $\gamma \in(\alpha, \delta)$ be a successor ordinal and we shall prove

$(*)_{3}\left(c_{\gamma, \alpha}^{*}{ }^{\wedge} \bar{d}_{\gamma, \alpha}^{*}\right)^{\wedge} \ldots \wedge\left(\bar{c}_{\gamma+n, \alpha}^{*}{ }^{\wedge} \bar{d}_{\gamma+n, \alpha}^{*}\right)$ and $\left(\bar{c}_{0}^{\alpha \wedge} \bar{d}_{0}^{\alpha}\right)^{\wedge} \ldots{ }^{\wedge}\left(\bar{c}_{n}^{\alpha \wedge} \bar{d}_{n}^{\alpha}\right)$ realize the same type over $A_{1} \cup A_{2, \gamma}$.

As $\delta$ is a limit ordinal this suffices by 4.7 (with $n=2, I_{0}$ a singleton, $I_{1}$ isomorphic to $\delta, I_{2}$ isomorphic to $\left.\omega\right)$. Now $\left(\bar{c}_{\gamma, \alpha}^{*}{ }^{\wedge} \bar{d}_{\gamma, \alpha}^{*}\right)^{\wedge} \ldots{ }^{\wedge}\left(\bar{c}_{\gamma+n-1, \alpha}^{*}{ }^{\wedge} \bar{d}_{\gamma+n-1, \alpha}^{*}\right)$ and $\left(\bar{c}_{0}^{\alpha \wedge} \bar{d}_{0}^{\alpha}\right)^{\wedge} \ldots{ }^{\wedge}\left(\bar{c}_{n-1}^{\alpha}{ }^{\wedge} \bar{d}_{n-1}^{\alpha}\right)$ realize the same type over $A_{1} \cup A_{2, \gamma}$ by the induction hypothesis.

Next by Clause (b) of the assumption $\operatorname{tp}\left(\bar{c}_{n}^{\alpha}, M \cup \bigcup\left\{\bar{c}_{m}^{\alpha}{ }^{\wedge} \bar{d}_{m}^{\alpha}: m<n\right\}\right)$ does not split over $B_{\alpha}$ hence $\operatorname{tp}\left(\bar{c}_{n}^{\alpha}, A \cup A_{1} \cup A_{2} \cup A_{3}\right)$ does not split over $B_{\alpha} \subseteq A$ by $(*)_{1}$.

Hence by the induction hypothesis $\left\langle\bar{c}_{\alpha}^{\prime \prime} \wedge \bar{d}_{\alpha}^{\prime \prime \prime}\right\rangle^{\wedge}\left\langle\bar{c}_{\beta, \alpha}^{*}{ }^{\wedge} d_{\beta, \alpha}^{*}: \beta \in[\alpha, \delta)\right\rangle^{\wedge}\left\langle\left(\bar{c}_{k}^{\alpha \wedge} \bar{d}_{k}^{\alpha}\right)\right.$ : $k<n\rangle$ is an indiscernible sequence over $A \cup \bar{c}_{n}^{\alpha}$, hence 
- $\left(\bar{c}_{\gamma, \alpha}^{*}{ }^{\wedge} \bar{d}_{\gamma, \alpha}^{*}\right)^{\wedge} \ldots{ }^{\wedge}\left(\bar{c}_{\gamma+n-1, \alpha}^{*} \hat{d}_{\gamma+n-1, \alpha}^{*}\right)^{\wedge} \bar{c}_{n}^{\alpha}$ and $\left(\bar{c}_{0}^{\alpha \wedge} \bar{d}_{0}^{\alpha}\right)^{\wedge} \ldots{ }^{\wedge}\left(\bar{c}_{\gamma+n-1, \alpha}^{*} \bar{d}_{\gamma+n-1, \alpha}^{*}\right)^{\wedge} \bar{c}_{n}^{\alpha}$ realize the same type over $A_{1} \cup A_{2, \gamma}$.

But by $\circledast_{1}$ clearly $\bar{c}_{n}^{\alpha}, \bar{c}_{\gamma+n, \alpha}^{*}$ realize the same type over $A_{1} \cup A_{2, \gamma}$ hence

- $\left(c_{\gamma, \alpha}^{*} \wedge \bar{d}_{\gamma, \alpha}^{*}\right)^{\wedge} \ldots{ }^{\wedge}\left(\bar{c}_{\gamma+n-1, \alpha}^{*} \wedge \bar{d}_{\gamma+n-1, \alpha}^{*}\right)^{\wedge} \bar{c}_{\gamma+n, \alpha}$ and $\left(\bar{c}_{0}^{\alpha \wedge} \bar{d}_{0}^{\alpha}\right)^{\wedge} \ldots \wedge\left(\bar{c}_{n-1}^{\alpha} \wedge \bar{d}_{n-1}^{\alpha}\right)^{\wedge} \bar{c}_{n}^{\alpha}$ realize the same type over $A_{1} \cup A_{2, \gamma}$.

We can choose $\bar{d}^{\prime}$ in $\mathfrak{C}$ such that $\left(\bar{c}_{\gamma, \alpha}^{*} \wedge \bar{d}_{\gamma, \alpha}^{*}\right)^{\wedge} \ldots{ }^{\wedge}\left(\bar{c}_{\gamma+n-1, \alpha}^{*}{ }^{\wedge} \bar{d}_{\gamma+n-1, \alpha}^{*}\right)^{\wedge}\left(\bar{c}_{\gamma+n, \alpha}^{*}{ }^{\wedge} \bar{d}^{\prime}\right)$ and $\left(\bar{c}_{0}^{\alpha \wedge} \bar{d}_{0}^{\alpha}\right)^{\wedge} \ldots{ }^{\wedge}\left(\bar{c}_{n-1}^{\alpha} \hat{d}_{n-1}^{\alpha}\right)^{\wedge}\left(\bar{c}_{n}^{\alpha \wedge} \bar{d}_{n}^{\alpha}\right)$ realize the same type over $A_{1} \cup A_{2, \gamma}$ so (to prove $\left.\circledast_{6}\right)$ it suffices to prove that $\bar{d}_{\gamma+n, \alpha}^{*}, \bar{d}^{\prime}$ realize the same type over $A_{1} \cup A_{2, \gamma+n} \cup$ $\bar{c}_{\gamma+n, \alpha}^{*}$.

Recall that $\left\langle\bar{c}_{\beta, \alpha}^{*} \hat{d}_{\beta, \alpha}^{*}: \beta \in[\alpha, \delta)\right\rangle^{\wedge}\left\langle\bar{c}_{n}^{\alpha \wedge} \bar{d}_{n}^{\alpha}: n<\omega\right\rangle$ is an indiscernible sequence, hence the sequences $\bar{c}_{\gamma+n-1, \alpha}^{*}{ }^{\wedge} \bar{d}_{\gamma+n-1, \alpha}^{*}{ }^{\wedge} \bar{c}_{\gamma+n, \alpha}^{*}{ }^{\wedge} \bar{d}_{\gamma+n, \alpha}^{*}$ and $\bar{c}_{\gamma+n-1, \alpha}^{*}{ }^{\wedge} \bar{d}_{\gamma+n-1, \alpha}^{*}{ }^{\wedge} \bar{c}_{n}^{\alpha \wedge} \bar{d}_{n}^{\alpha}$ realize the same type.

By the two previous sentences and the transitivity of the equality of types $\bar{d}_{\gamma+n, \alpha}^{*}, \bar{d}^{\prime}$ realize the same type over $\left(\bar{c}_{\gamma+n-1, \alpha}^{*}{ }^{{ }^{*}} \bar{d}_{\gamma+n-1, \alpha}^{*}\right)^{\wedge} \bar{c}_{\gamma+n, \alpha}^{*}$, but by Clause $(\mathrm{e})^{-}$of Definition 3.5 which apply by Clause (b) of the assumption and $\circledast_{5}$ above we have $\operatorname{tp}\left(\bar{d}_{\gamma+n, \alpha}^{*}, \bar{c}_{\gamma+n, \alpha}^{*}+\bar{d}_{\gamma+n-1, \alpha}^{*}\right) \vdash \operatorname{tp}\left(\bar{d}_{\gamma+n, \alpha}^{*}, A_{1}+A_{2, \gamma+n}+\bar{c}_{\gamma+n, \alpha}^{*}\right)$ so we are done.]

Definition 4.14. 1) We say that the strict $(\kappa,<\theta)$-decompositions $\left\langle\left(\bar{c}_{\varepsilon}^{\prime}, \bar{d}_{\varepsilon}^{\prime}\right)\right.$ : $\varepsilon<\delta\rangle,\left\langle\left(\bar{c}_{\varepsilon}^{\prime \prime}, \bar{d}_{\varepsilon}^{\prime \prime}\right): \varepsilon<\delta\right\rangle$ over $M$ are equivalent over $B \in[M]^{<\lambda} \underline{\text { when for some }}$ automorphism $f$ of $M$ over $B$ for every $n$ and $\varepsilon_{0}<\ldots<\varepsilon_{n-1}<\delta, f$ maps the type $\operatorname{tp}\left(\left(\bar{c}_{\varepsilon_{\delta}}^{\prime} \wedge \bar{d}_{\varepsilon_{\delta}}^{\prime}\right)^{\wedge} \ldots \wedge\left(\bar{c}_{\varepsilon_{n-1}}^{\prime} \wedge \bar{d}_{\varepsilon_{n-1}}^{\prime}\right), M\right)$ to the type $\operatorname{tp}\left(\left(\bar{c}_{\varepsilon_{0}}^{\prime \prime} \wedge \bar{d}_{\varepsilon_{0}}^{\prime \prime}\right)^{\wedge} \ldots{ }^{\wedge}\left(\bar{c}_{\varepsilon_{n-1}}^{\prime \prime}{ }^{\wedge} \bar{d}_{\varepsilon_{n-1}}^{\prime \prime}\right), M\right)$ and $\ell g\left(\bar{c}_{\varepsilon_{0}}^{\prime}\right)=\ell g\left(\bar{c}_{\varepsilon_{0}}^{\prime \prime}\right), \ell g\left(\bar{d}_{\varepsilon_{0}}^{\prime}\right)=\ell g\left(\bar{d}_{\varepsilon_{0}}^{\prime \prime}\right)$.

2) In part (1) we say "weakly equivalent over $B$ " when for every $\zeta<\kappa$ and $\bar{b}^{\prime} \in{ }^{\zeta} M$ there is $\bar{b}^{\prime \prime} \in \zeta^{\zeta} M$ and vice versa and elementary mapping $f$ such that: $f \supseteq \operatorname{id}_{B}, f\left(\bar{b}^{\prime}\right)=\bar{b}^{\prime \prime}$ and $f\left(\bar{c}_{\varepsilon}^{\prime}\right)=f\left(\bar{c}_{\varepsilon}^{\prime \prime}\right), f\left(\bar{d}_{\varepsilon}^{\prime}\right)=f\left(\bar{d}_{\varepsilon}^{\prime \prime}\right)$ for $\varepsilon<\delta$.

3) If $B=\emptyset$ then we may omit it.

Claim 4.15. 1) If $M$ is $\kappa$-saturated of cardinality $\lambda$ and $B \in[M]^{<\lambda} \underline{\text { then }}$ the number of strict $(\lambda, \kappa,<\theta)$-decompositions $\left\langle\left(\bar{c}_{n}, \bar{d}_{n}\right): n<\omega\right\rangle$ over $(M, B)$ such that $\ell g\left(\bar{d}_{n}\right)>0$ up to weak equivalence or when $\ell g\left(\bar{d}_{n}\right)=0$ up to equivalence over $B$ is $\leq \lambda$, see 4.14 .

2) For $M, B$ as above, two strict $(\kappa,<\theta)$-decompositions are equivalent when they are weakly equivalent above $(M, B)$ and $\lambda=\kappa$.

3) if $\left\langle\bar{c}_{n}, \bar{d}_{n}\right): n\langle\omega\rangle$ is a $(\kappa,<\theta)$-decomposition over $(M, B)$ so $B \in[M]^{\kappa}$ and $C=\cup\left\{\operatorname{rang}\left(\bar{c}_{n} \wedge \bar{d}_{n}\right): n<\omega\right\} \cup B \underline{\text { then }} M_{[C]}$ is a sequence homogeneous model.

Proof. 1) First, if $\ell g\left(\bar{d}_{n}\right)=0$, by $1.2(4)$, that is [Sh:783, 5.26], however in the present case $\lambda$ is measurable hence strongly inaccessible so 4.15) (1) is easy. That is, fixing $A, B$, also the number of $\alpha<\lambda$ and $p\left(\bar{x}_{\alpha}\right) \in \mathbf{S}(M)$ not splitting over $B$ is $\leq \lambda$ by 1.2. The case for weakly equivalent holds as $\lambda$ is strongly inaccessible.

2) Let $\left\langle\left(\bar{c}_{n}^{\prime}, \bar{d}_{n}^{\prime}\right): n<\omega\right),\left\langle\left(\bar{c}_{n}^{\prime \prime}, \bar{d}_{n}^{\prime \prime}\right): n<n\right\rangle$ be two strict $(\kappa,<\theta)$-decompositions over $M$. As a $\lambda$-sequence-homogeneous model of cardinality $\lambda$ is determined up to isomorphisms by the set of complete types of finite tuples in it (by [KM67] or see Sh:88r, §2]) by part (3) it suffices to show:

$(*)_{1}$ for every $\bar{b}^{\prime} \in{ }^{\omega>} M$ there are $\bar{b}^{\prime \prime} \in{ }^{\omega>} M$ and an elementary mapping $f$ such that $f\left(\bar{b}^{\prime}\right)=\bar{b}^{\prime \prime}, f \supseteq \operatorname{id}_{B}$ and $\varepsilon<\delta \Rightarrow f\left(\bar{c}_{\varepsilon}^{\prime}\right)=\bar{c}_{\varepsilon}^{\prime \prime}$, 
$(*)_{2}$ for every $\bar{b}^{\prime \prime} \in \omega>M$ there are $\bar{b}^{\prime} \in \omega>M$ there $f$ as above.

By symmetry it suffices to prove $(*)_{1}$. Given $\bar{b}^{\prime}$ let $B^{*}=B \cup \bar{b}$. Let $\delta<\kappa$ be a finite ordinal. We choose $\left(\bar{c}_{\varepsilon}^{*}, \bar{d}_{\varepsilon}^{*}\right)$, a pair of sequences from $M$ such that $\left\langle\bar{c}_{\varepsilon}^{\prime \wedge} \bar{d}_{\varepsilon}^{\prime}\right\rangle^{\wedge}\left\langle\bar{c}_{n}^{\prime} \wedge \bar{d}_{n}^{\prime}: n<\omega\right\rangle$ is an indiscernible sequence over $B^{\prime} \cup \bigcup\left\{\bar{c}_{\zeta}^{* \wedge} \bar{d}_{\zeta}^{*}: \zeta<\varepsilon\right\}$ by $3.5(\mathrm{f})$ with $\cup\left\{\bar{c}_{\zeta}^{\prime}{ }^{\wedge} \bar{d}_{\zeta}^{\prime}: \zeta<\varepsilon\right\} \cup B^{\prime}, M,\left\langle\left(\bar{c}_{\varepsilon}^{\prime}, \bar{d}_{\varepsilon}^{\prime}\right): \varepsilon<\delta\right\rangle,\left(\bar{c}_{\varepsilon}^{\prime}, \bar{d}_{\varepsilon}^{\prime}\right)$ here standing for $A, M,\left\langle\left(\bar{c}_{t}, \bar{d}_{t}\right): t \in J\right\rangle,(\bar{c}, \bar{d})$ there and similarly $\left(\bar{c}_{\varepsilon}^{\prime \prime}, \bar{d}_{\varepsilon}^{\prime \prime}\right)$ for $\varepsilon<\delta$ with $B^{\prime \prime}:=B$.

Now as $M$ is $\kappa$-saturated we can find $\bar{b}^{\prime \prime} \in \ell g\left(\bar{b}^{\prime}\right) M$ such that the sequences $\bar{b}^{\prime \wedge} \bar{c}^{\prime \wedge} \bar{d}^{\prime \wedge}\left(\ldots{ }^{\wedge} \bar{c}_{\varepsilon}^{\prime \wedge} \ldots\right)_{\varepsilon<\delta}$ and $\bar{b}^{\prime \prime \wedge} \bar{c}^{\prime \prime \wedge} \bar{d}^{\prime \prime \wedge}\left(\ldots{ }^{\wedge} \bar{c}_{\varepsilon}^{\prime \prime \wedge} \ldots\right)_{\varepsilon<\delta}$ realize the same type over $A$. We can finish by $3.5(1)(\mathrm{e})$ as in proof of 4.13

3) Let $g$ be the identity mapping on $\cup\left\{\operatorname{Rang}\left(\bar{c}_{n}{ }^{\wedge} \bar{d}_{n}\right): n<\omega\right\} \cup B$.

Let

$(*)_{1} \quad \mathscr{F}$ be the set of $f$ such that:

- $f$ is an elementary mapping

- $\operatorname{Dom}(f) \subseteq M$ has cardinality $<\kappa$

- $\operatorname{Rang}(f) \subseteq M$

- $f \cup g$ is an elementary mapping.

It suffices to prove $(*)_{1}-(*)_{4}$ below:

$(*)_{1} \quad \mathscr{F} \neq \emptyset$.

[Why? As $f=\operatorname{id}_{B}$ belongs to $\mathscr{F}$.]

$(*)_{2} f \in \mathscr{F}$ iff $f^{-1} \in \mathscr{F}$

$(*)_{3} \mathscr{F}$ is closed under union of increasing chains of length $<\lambda$.

[Why? Just check.]

$(*)_{4}$ if $f \in \mathscr{F}, \ell \in\{1,2\}$ and $a_{\ell} \in M$ then for some $a_{3-\ell} \in M$ we have $f \cup$ $\left\{\left(a_{1}, a_{2}\right)\right\} \in \mathscr{F}$.

Why? By symmetry, without loss of generality $\ell=1$. Let $\delta$ be a limit ordinal $<\kappa$. We choose $\bar{c}_{\varepsilon}^{\prime}, \bar{d}_{\varepsilon}^{\prime}$ sequences from $M$ of length $\ell g\left(\bar{c}_{0}\right), \ell g\left(\bar{d}_{0}\right)$ respectively by induction on $\varepsilon<\delta$, by applying 3.5 (f) to $M,\left\langle\left(\bar{c}_{n}, \bar{d}_{n}\right): n<\omega\right\rangle$ and $B_{\varepsilon}=B \cup$ $\operatorname{Dom}(f) \cup\{a\} \cup \operatorname{Rang}(f) \cup \bigcup\left\{\bar{c}_{\zeta}^{\prime} \wedge \bar{d}_{\zeta}^{\prime}: \zeta<\varepsilon\right\}$ and get $\bar{c}_{\varepsilon}, \bar{d}_{\varepsilon}$ from $M$ as there.

$(*)_{4.1}\left\langle\bar{c}_{\varepsilon}^{\prime} \bar{d}_{\varepsilon}^{\prime}: \varepsilon<\delta\right\rangle^{\wedge}\left\langle\left(\bar{c}_{n}{ }^{\wedge} \bar{d}_{n}\right): n<\omega\right\rangle$ is an indiscernible sequence over $B_{0}$.

This implies that, letting $B_{*}=\cup\left\{\bar{c}_{\varepsilon}^{\prime} \bar{d}_{\varepsilon}^{\prime}: \varepsilon<\delta\right\}$ :

$(*)_{4.2} f \cup g \cup \operatorname{id}_{B_{*}}$ is an elementary mapping.

As $M$ is $\kappa$-saturated

$(*)_{4.3}$ there is $a_{2} \in M$ such that $f \cup \operatorname{id}_{\bar{c}^{\wedge} \bar{d}} \cup\left\{\left(a_{1}, a_{2}\right)\right\}$ is an elementary mapping.

So we can prove as in the proof of 4.13

$(*)_{4.4} f \cup\left\{\left(a_{1}, a_{2}\right)\right\} \in \mathscr{F}$.

So we are done. 
Clearly 4.13 is a step forward. Now we prove the generic pair conjecture; instead of assuming that the cardinality $\lambda$ is measurable we can restrict $T$.

Toward this

Definition 4.16. We say that the triple $\mathbf{m}=(M, N, \mathscr{A})$ is a $(\lambda, \kappa,<\theta)$-system $\underline{\text { when }}(\lambda \geq \kappa \geq \theta=\operatorname{cf}(\theta)>|T|$ and) it satisfies clauses (a)-(d) below, and say the triple is a full $(\lambda, \kappa,<\theta)$-system when it satisfies clauses (a)-(i) below where:

$\boxplus$ (a) $\quad M$ is $\kappa$-saturated of cardinality $\lambda$,

(b) $\quad M \prec N \prec \mathfrak{C}$ and $N$ has cardinality $\lambda$,

(c) $\mathscr{A}_{\mathbf{m}}$ is a set of cardinality $\lambda$ of objects $\mathbf{p}$ such that:

$(\alpha) \mathbf{p}$ is of the form $\left\langle\left(\bar{c}_{\varepsilon}, \bar{d}_{\varepsilon}\right): \varepsilon<\lambda^{+}\right\rangle=\left\langle\left(\bar{c}_{\varepsilon}[\mathbf{p}], \bar{d}_{\varepsilon}[\mathbf{p}]\right): \varepsilon<\lambda^{+}\right\rangle$,

(ß) $\bar{c}_{0} \wedge \bar{d}_{0} \subseteq N$,

$(\gamma)\left\langle\left(\bar{c}_{\varepsilon}, \bar{d}_{\varepsilon}\right): \varepsilon<\lambda^{+}\right\rangle$is a strict $(\kappa,<\theta)$-decomposition over $M$,

(d) $\mathscr{A}_{\mathbf{m}}$ is partially ordered by: $\mathbf{p} \leq_{\mathbf{m}} \mathbf{q}$ iff

- $\bar{c}_{0}[\mathbf{p}] \unlhd \bar{c}_{0}[\mathbf{q}]$ and $\bar{d}_{0}[\mathbf{p}] \unlhd \bar{d}_{0}[\mathbf{q}]$,

- for every large enough $\varepsilon<\lambda^{+}, \bar{c}_{\varepsilon}[\mathbf{p}] \unlhd \bar{c}_{\varepsilon}[\mathbf{q}], \bar{d}_{\varepsilon}[\mathbf{p}] \unlhd \bar{d}_{\varepsilon}[\mathbf{q}]$,

(e) $\mathscr{A}_{\mathbf{m}}$ is closed under union of $<\theta$ increasing chains of length $<\theta$,

(f) if $\mathbf{p} \in \mathscr{A}_{\mathbf{m}}$ and $\bar{d} \in{ }^{\theta>} N$ then for some $\mathbf{q} \in \mathscr{A}$ above $\mathbf{p}$ we have $\operatorname{Rang}(\bar{d}) \subseteq \operatorname{Rang}\left(\bar{d}_{0}[\mathbf{q}]\right)$

(g) $N$ is saturated,

(h) up to really equivalence every possible $\mathbf{p}$ in Clause (c) occurs (i.e. is represented in $\mathscr{A})$, where $\mathbf{p}, \mathbf{q}$ are really equivalent when they are as in (c) and $\left(\bar{c}_{\mathbf{p}, 0}, \bar{d}_{\mathbf{p}, 0}\right)=\left(\bar{c}_{\mathbf{q}, 0}, \bar{d}_{\mathbf{q}, 0}\right)$

(i) there is $\mathbf{q}_{2} \in \mathscr{A}_{\mathbf{m}}$ such that $\mathbf{p}_{2} \leq_{\mathbf{m}} \mathbf{q}_{2}$ and $\mathbf{q}_{2}, \mathbf{q}_{1}$ are equivalent as witnessed by $f$, when:

( $\alpha) \quad \mathbf{p}_{1} \leq_{\mathbf{m}} \mathbf{q}_{1}$ so both are from $\mathscr{A}_{\mathbf{m}}$,

( $\beta) \quad \mathbf{p}_{2} \in \mathscr{A}_{\mathbf{m}}$ is really equivalent to $\mathbf{p}_{1}$,

$(\gamma) \quad f \in \operatorname{aut}(M)$ maps $\mathbf{p}_{1}$ to $\mathbf{p}_{2}$.

Definition 4.17. 1) Let $\mathrm{BP}=\mathrm{BP}_{\lambda, \kappa,<\theta}$ be the set of $(\lambda, \kappa,<\theta)$-systems.

2) If $\left(M_{\ell}, N_{\ell}, \mathscr{A}_{\ell}\right)$ is a $(\lambda, \kappa,<\theta)$-system for $\ell=1,2$ we say $\left(M_{2}, N_{2}, \mathscr{A}_{2}\right)$ is above $\left(M_{1}, N_{1}, \mathscr{A}_{1}\right)$ or $\left(M_{1}, N_{1}, \mathscr{A}_{1}\right) \leq_{\mathrm{BP}_{\lambda, \kappa,<\theta}}\left(M_{2}, N_{2}, \mathscr{A}_{2}\right)$ when $M_{1}=M_{2}, N_{1} \prec N_{2}$ and $\mathscr{A}_{1} \subseteq \mathscr{A}_{2}$.

3) We may write just $\mathrm{BP}, \leq_{\mathrm{BP}} \underline{\text { when }}(\lambda, \kappa,<\theta)$ is clear from the context.

4) If $\mathbf{m} \in \mathrm{BP}$ and $\mathbf{p} \in \mathscr{A}_{\mathbf{m}}$, we say that $B$ is a base of $\mathbf{p}$ when $B \subseteq M_{\mathbf{m}}$ has cardinality $<\kappa$ and $\mathbf{p}$ is a strict $(\kappa,<\theta)$-decomposition over $(M, B)$.

Claim 4.18. Assume $\kappa=\lambda=\lambda^{<\lambda}$ (see Definition 4.10).

1) If $M \prec \mathfrak{C}$ is $\kappa$-saturated of cardinality $\lambda$ then there is a pair $(N, \mathscr{A})$ such that $(M, N, \mathscr{A})$ is a $(\lambda, \kappa,<\theta)$-system.

2) If $\left(M, N_{1}, \mathscr{A}_{1}\right)$ is a $(\lambda, \kappa,<\theta)$-system and $N_{2} \prec \mathfrak{C}$ and $\left\|N_{2}\right\|=\lambda$ then there is $a$ pair $\left(N_{3}, \mathscr{A}_{3}\right)$ such that $\left(M, N_{3}, \mathscr{A}_{3}\right)$ is a $(\lambda, \kappa,<\theta)$-system above $\left(M, N_{1}, \mathscr{A}_{1}\right)$ and $N_{2} \prec N_{3}$.

3) If $\left\langle\left(M, N_{\varepsilon}, \mathscr{A}_{\varepsilon}\right): \varepsilon<\delta\right\rangle$ is an increasing sequence of $(\lambda, \kappa,<\theta)$-systems and $\delta$ is a limit ordinal $<\lambda^{+}$then the union, $\left(M, \bigcup_{\alpha<\delta} N_{\alpha}, \bigcup_{\alpha<\delta} \mathscr{A}_{\alpha}\right)$ is a $(\lambda, \kappa,<\theta)$-system which is a least upper bound of $\left\{\left(M, N_{\varepsilon}, \mathscr{A}_{\varepsilon}\right): \varepsilon<\delta\right\}$. 
4) If in part (3) we have $\operatorname{cf}(\delta)=\lambda$ and each is full, then so is the union.

Proof. 1) Let $\mathbf{m}=(M, M, \emptyset)$ and check.

2) Let $N_{3} \prec \mathfrak{C}$ be such that $N_{1} \cup N_{2} \prec N_{3}, N_{3}$ is saturated of cardinality $\lambda$ and use $\left(M, N_{3}, \mathscr{A}_{1}\right)$.

3) Easy.

4) Easy.

Claim 4.19. 1) We have:

(a) $\leq_{\mathrm{BP}}$ is a partial order on $\mathrm{BP}$,

(b) any $\leq_{\mathrm{BP}}$-increasing sequence of length $<\lambda^{+}$has an upper bound,

$(b)^{+}$moreover if $\left\langle\left(M, N_{\alpha}, \mathscr{A}_{\alpha}\right): \alpha<\delta\right\rangle$ is $\leq_{\mathrm{BP}}$-increasing then $\left(M, \cup\left\{N_{\alpha}: \alpha<\right.\right.$ $\left.\delta\}, \cup\left\{\mathscr{A}_{\alpha}: \alpha<\delta\right\}\right)$ is a least $\leq_{\mathrm{BP}}$-upper bound,

(c) $\mathrm{BP}$ is not empty, moreover for every $M \in \mathrm{EC}_{\lambda, \lambda}(T)$ there is $\mathbf{m} \in \mathrm{BP}$ such that $M_{\mathbf{m}}=M=N_{\mathbf{m}}, \mathscr{A}_{\mathbf{m}}=\emptyset$.

2) If $\mathbf{m}$ is $a(\lambda, \kappa,<\theta)$-system then:

(a) $\leq_{\mathbf{m}}$ is a partial order of $\mathscr{A}_{\mathbf{m}}$,

(b) if also $\mathbf{n}$ is a $(\lambda, \kappa,<\theta)$-system and $\mathbf{m} \leq_{\mathrm{BP}} \mathbf{n}$ then $\leq_{\mathbf{m}}=\leq_{\mathbf{n}} \mid \mathscr{A}_{\mathbf{m}}$,

(c) if $\delta$ as a limit ordinal $<\theta$ and $\mathbf{p}_{\alpha} \in \mathscr{A}_{\mathbf{m}}$ for $\alpha<\delta$ is $\leq_{\mathbf{m}}$-increasing with $\alpha$ then there is $\mathbf{n} \in \mathrm{BP}$ such that $\mathbf{m} \leq_{\mathrm{BP}} \mathbf{n}$ and $\leq_{\mathbf{n}}$-upper bound of $\left\{\mathbf{p}_{\alpha}: \alpha<\delta\right\}$,

(d) if $\mathbf{p} \in \mathscr{A}_{\mathbf{m}}$ and $\bar{d} \in{ }^{\theta>} N$ then there are $a(\lambda, \kappa,<\theta)$-system $\mathbf{n}$ satisfying $\mathbf{m} \leq_{\mathrm{BP}} \mathbf{n}$ and $\mathbf{q} \in \mathscr{A}_{\mathbf{n}}$ such that $\mathbf{p} \leq_{\mathbf{n}} \mathbf{q}$ and $\bar{d} \subseteq \operatorname{Rang}\left(\bar{d}_{0}^{\mathbf{q}}\right)$,

(e) if $\mathbf{p}_{1}, \mathbf{p}_{2} \in \mathscr{A}_{\mathbf{m}}$ are equivalent and $\mathbf{p}_{1} \leq_{\mathbf{m}} \mathbf{q}_{2}$ then for some $\mathbf{n} \in \mathrm{BP}$ we have $\mathbf{m} \leq_{\mathrm{BP}} \mathbf{n}$ and for some $\mathbf{q}_{2} \in \mathscr{A}_{\mathbf{n}}$ equivalent to $\mathbf{q}_{1}$ we have $\mathbf{p}_{1} \leq_{n} \mathbf{q}_{2}$.

3) If $(M, N, \mathscr{A})$ is a $(\lambda, \kappa,<\theta)$-system then there is a full $(\lambda, \kappa,<\theta)$-system above it.

Proof. 1) Clause (a) holds easily by checking $4.17(2)$. Clauses (b), (b) ${ }^{+}$holds easily by $4.18(3)$, and for Clause (c) use $(M, M, \emptyset)$.

2) Why? Clauses (a),(b): Easy.

Clause (c): For $\alpha<\beta<\delta$ let $\zeta=\zeta(\alpha, \beta)<\lambda^{+}$be such that:

- if $\varepsilon \in\left[\zeta, \lambda^{+}\right)$then $\bar{c}_{\varepsilon}^{\mathbf{p}_{\alpha}} \unlhd \bar{c}_{\varepsilon}^{\mathbf{p}_{\beta}}, \bar{d}_{\varepsilon}^{\mathbf{p}_{\alpha}} \unlhd \bar{d}_{\varepsilon}^{\mathbf{p}_{\beta}}$.

Let $\zeta(*)=\sup \{\zeta(\alpha, \beta)+2: \alpha<\beta<\delta\}$, so necessarily $\zeta(*)<\lambda^{+}$. Clearly if $\zeta \in\left[\zeta(*), \lambda^{+}\right) \cup\{0\}$ then $\left(\bar{c}_{\zeta}^{\mathbf{p}_{\alpha}}: \alpha<\delta\right\rangle$ is $\unlhd$-increasing and also the sequence $\left\langle d_{\zeta}^{\mathbf{p}_{\alpha}}: \alpha<\delta\right\rangle$ is $\unlhd$-increasing.

We choose $\bar{c}_{0}=\cup\left\{\bar{c}_{0}^{\mathbf{p}_{\alpha}}: \alpha<\delta\right\}, \bar{d}_{0}=\cup\left\{\bar{d}_{0}^{\mathbf{p}_{\alpha}}: \alpha<\delta\right\}$ and for $\varepsilon<\lambda^{+}$let $\bar{c}_{1+\varepsilon}=\cup\left\{\bar{c}_{\zeta(*)+\varepsilon}^{\mathbf{p}_{\alpha}}: \alpha<\delta\right\}$ and $\bar{d}_{1+\varepsilon}=\cup\left\{\bar{d}_{\zeta(*)+\varepsilon}^{\mathbf{p}_{\alpha}}: \alpha<\delta\right\}$.

Now easily

- $\mathbf{p}=\left\langle\left(\bar{c}_{\varepsilon}, \bar{d}_{\varepsilon}\right): \varepsilon\left\langle\lambda^{+}\right\rangle\right.$is well defined and satisfies the demand in $4.16(c)(\alpha)+$ $(\beta)+(\gamma)$.

[Why? By Claim 4.13,]

So we define $\mathbf{n}$ as $\left(M, N_{\mathbf{m}},\{\mathbf{p}\} \cup \mathscr{A}_{\mathbf{m}}\right)$, easily 
- $\mathbf{n} \in \mathrm{BP}$ and $\mathbf{m} \leq \mathbf{n}$.

Lastly, $\alpha<\delta \Rightarrow \mathbf{p}_{\alpha} \leq_{\mathbf{n}} \mathbf{p}$ as witnessed by $\zeta(*) \times \omega$. So we are done proving clause (c).

Clause (d):

Easy. By Clause (D) of Definition 4.10 of " $(\lambda, \kappa,<\theta)$ is $T$-good" which holds by Hypothesis 4.12

Clause (e):

By the definition of "equivalence" in Definition 4.14 (and 4.16(h)).

3) We fix a $(\lambda, \kappa,<\theta)$-system $\left(M, N_{*}, \mathscr{A}_{*}\right)$. We now shall choose $\mathbf{m}_{i} \in \mathrm{BP}, \leq_{\mathrm{BP}^{-}}$ increasing by induction on $i \leq \lambda$ and

$\odot_{1}$ for $i=0: \mathbf{m}_{0}$ is $\left(M, N_{*}, \mathscr{A}_{*}\right)$

$\odot_{2}$ for limit $i<\lambda, \mathbf{m}_{i}$ is a $\leq_{\mathrm{BP}}$-upper bound of $\left\langle\mathbf{m}_{j}: j<i\right\rangle$, in fact the union.

[Why? Possible by clause $(b)^{+}$of part (1).]

By bookkeeping

$\odot_{3}$ if $\left\langle\mathbf{p}_{\alpha}: \alpha<\delta\right\rangle$ is $\leq_{\mathbf{m}_{j}}$-increasing, $\delta$ a limit ordinal $<\theta$ and $j<\lambda$, then for some $i \in(j, \lambda)$ the sequence has an upper bound by $\leq_{\mathbf{m}_{i+1}}$.

[Why? By Clause (c) of part (2).]

$\odot_{4}$ if $\mathbf{p} \in \mathscr{A}_{\mathbf{m}_{j}}$ and $\bar{d} \in{ }^{\theta>} N_{\mathbf{m}_{j}}$ and $j<\lambda \underline{\text { then }}$ for some $i \in(j, \lambda)$, there is $\mathbf{q} \in \mathscr{A}_{\mathbf{m}_{i+1}}$ such that $\mathbf{p} \leq \mathbf{m}_{i+1} \mathbf{q}$ and $\operatorname{Rang}(\bar{d}) \subseteq \operatorname{Rang}\left(\bar{d}_{0}^{\mathbf{q}}\right)$.

[Why? By Clause (d) of part (2).]

$\odot_{5}$ if $j<\lambda$ and $\mathbf{p}_{1}, \mathbf{q}_{1}, \mathbf{p}_{2}, f$ satisfy Clauses $(\alpha)-(\varepsilon)$ of 4.16 (i) with $\mathbf{m}_{j}$ here standing for $\mathbf{m}$ there then for some $i \in(j, \lambda)$ there is $\mathbf{q}_{2} \in \mathscr{A}_{\mathbf{m}_{i+1}}$ such that $\mathbf{p}_{2} \leq_{\mathbf{m}_{i+1}} \mathbf{q}_{2}$ and $\mathbf{q}_{1}, \overline{\mathbf{q}_{2}}$ are equivalent as witnessed by $f$.

[Why? Just think.]

So we can carry the induction. Now $\mathbf{m}_{\lambda}$ is as required.

Theorem 4.20. Assume $(\lambda, \kappa,<\theta)=(\lambda, \lambda,<\lambda)$ so is a T-good triple, see 4.10. 4.12.

1) If $\lambda^{+}=2^{\lambda},\left\langle M_{\alpha}: \alpha<\lambda^{+}\right\rangle$is an $\prec$-increasing continuous sequence of members of $\operatorname{EC}_{\lambda}(T), M_{\alpha}$ saturated if $\alpha$ is non-limit and $M=\cup\left\{M_{\alpha}: \alpha<\lambda^{+}\right\}$is saturated then for some club (= closed unbounded subset) $E$ of $\lambda^{+}$for any $\alpha<\beta<\delta \in E$ and $\alpha, \beta$ are non-limit or are from $S_{\lambda}^{\lambda^{+}}$, the pairs $\left(M_{\delta}, M_{\alpha}\right)$ and $\left(M_{\delta}, M_{\beta}\right)$ are isomorphic. 2) If $\mathbf{m}_{\ell}=\left(M_{\ell}, N_{\ell}, \mathscr{A}_{\ell}\right)$ is a full $(\lambda, \lambda,<\lambda)$-system for $\ell=1,2$ then $\left(N_{1}, M_{1}\right) \cong$ $\left(N_{2}, M_{2}\right)$ that is there is an isomorphism $f$ from $N_{1}$ onto $N_{2}$ mapping $M_{1}$ onto $M_{2}$.

Proof. 1) By part (2), noting that $M_{\alpha}, M_{\beta}$ are saturated and recallihng 4.19 and its proof.

2) We define the set AP of approximation:

$(*)_{1} \quad \mathrm{AP}$ is the set of triples $\mathbf{h}=\left(\mathbf{p}_{1}, B_{1}, \mathbf{p}_{2}, B_{2}, f\right)=\left(p_{1}[\mathbf{h}], B_{2}[\mathbf{h}], p_{2}[\mathbf{h}], B_{2}[\mathbf{h}], f[\mathbf{h}]\right)$ satisfying:

(a) $\mathbf{p}_{\ell} \in \mathscr{A}_{\ell}$ for $\ell=1,2$,

(b) $B_{\ell} \in\left[M_{\ell}\right]^{<\lambda}$ is a base for $\mathbf{p}_{\ell}$, see $4.17(4)$, 
(c) $f$ is an elementary mapping which maps $B_{1}$ onto $B_{2}$ such that $\operatorname{Dom}(f)=$ $B_{1}$,

(d) there is an isomorphism $f^{+}$from $M_{1}$ onto $M_{2}$ extending $f$ such that: if $\alpha_{0}<\ldots<\alpha_{n-1}<\lambda^{+}$then $f^{+} \operatorname{maps} \operatorname{tp}\left(\left(\bar{c}_{\mathbf{p}_{1}, \alpha_{1}}{ }^{\wedge} \bar{d}_{\mathbf{p}_{1}, \alpha_{1}}\right)^{\wedge} \ldots{ }^{\wedge}\left(\bar{c}_{\mathbf{p}_{1}, \alpha_{n-1}}{ }^{\wedge} \bar{d}_{\mathbf{p}_{1}, \alpha_{n-1}}\right), M_{1}\right)$ onto $\operatorname{tp}\left(\left(\bar{c}_{\mathbf{p}_{2}, \alpha_{0}} \hat{d}_{\mathbf{p}_{2}, \alpha_{0}}\right)^{\wedge} \ldots{ }^{\wedge}\left(\bar{c}_{\mathbf{p}_{2}, \alpha_{n-1}}{ }^{\wedge} \bar{d}_{\mathbf{p}_{2}, \alpha_{n-1}}\right), M_{2}\right)$.

$(*)_{2}$ we define the two-place relation $\leq_{\mathrm{AP}}$ by: $\mathbf{h}_{1} \leq_{\mathrm{AP}} \mathbf{h}_{2}$ iff

(a) both are in AP,

(b) $\mathbf{p}_{\ell}\left[\mathbf{h}_{1}\right] \leq_{\mathbf{m}_{\ell}} \mathbf{p}_{\ell}\left[\mathbf{h}_{2}\right]$ for $\ell=1,2$,

(c) $B_{\ell}\left[\mathbf{h}_{1}\right] \subseteq B_{\ell}\left[\mathbf{h}_{2}\right]$,

(d) $f_{1}\left[\mathbf{h}_{1}\right] \subseteq f\left[\mathbf{h}_{2}\right]$

Obviously

$(*)_{3} \leq$ AP partially ordered AP.

Also

$(*)_{4}$ if $\delta<\lambda$ is a limit ordinal and $\left\langle\mathbf{h}_{\alpha}: \alpha<\delta\right\rangle$ is $\leq_{\mathrm{AP}}$-increasing, then this sequence has an $\leq_{\mathrm{AP}}$-upper bound.

[Why? As in the proof of 4.19 (2)(c).]

$(*)_{5}$ if $\mathbf{h} \in \mathrm{AP}$ and $a \in M_{1}$ then there is $\mathbf{h}^{\prime} \in \mathrm{AP}$ such that $\mathbf{h} \leq_{\mathrm{AP}} \mathbf{h}_{1}^{\prime}$ and $a \in B_{\mathbf{p}_{\ell}\left[\mathbf{h}_{1}^{\prime}\right]}$.

[Why? Let $a_{1}=a$ and let $f^{+} \supseteq f_{\mathbf{h}}$ be as in $(*)_{1}(d)$ and let $a_{2} \in M_{2}$ realize $f^{+}\left(\operatorname{tp}\left(a_{1}, B_{\mathbf{p}_{1}[\mathbf{h}]}\right)\right)$.

Let $B_{\ell}^{\prime}=\left(B_{\mathbf{p}_{\ell}[\mathbf{h}]} \cup\left\{a_{\ell}\right\}\right)$ and let $\mathbf{p}_{\ell}^{\prime} \in \mathscr{A}_{\mathbf{m}_{\ell}}$ be defined by: $\left(\bar{c}_{\varepsilon}\left[\mathbf{p}_{\ell}^{\prime}\right], \bar{d}_{\varepsilon}\left[\mathbf{p}_{\ell}^{\prime}\right]\right)=$ $\left(\bar{c}_{\varepsilon}\left[\mathbf{p}_{\ell}\left[\mathbf{h}_{1}\right], \bar{d}_{\varepsilon}\left[\mathbf{p}_{\ell}[\mathbf{h}]\right)\right.\right.$.

Lastly, let $\mathbf{h}^{\prime}=\left(\mathbf{p}_{1}^{\prime}, B_{1}^{\prime}, \mathbf{p}_{2}^{\prime}, B_{2}^{\prime}, f_{\mathbf{h}} \cup\left\{\left(a_{1}, a_{2}\right)\right\}\right)$.]

$(*)_{6}$ If $\mathbf{h} \in \mathrm{AP}$ and $a \in M_{2}$ then there is $\mathbf{h}^{\prime} \in \mathrm{AP}$ such that $\mathbf{h} \leq_{\mathrm{AP}} \mathbf{h}^{\prime}$ and $a \in B_{2}\left[\mathbf{h}^{\prime}\right]$.

[Why? Like $(*)_{5}$.]

$(*)_{7}$ if $\mathbf{h} \in \mathrm{AP}$ and $d \in N_{1}$ then for some $\mathbf{h}^{\prime} \in \mathrm{AP}$ we have $\mathbf{h} \leq_{\mathrm{AP}} \mathbf{h}^{\prime}$ and $d \in \operatorname{Rang}\left(\bar{d}_{0}\left[\mathbf{p}_{1}\left[\mathbf{h}^{\prime}\right]\right]\right)$.

[Why? There is $\mathbf{q}_{1} \in \mathscr{A}_{\mathbf{m}_{1}}$ such that $\mathbf{p}_{1}[\mathbf{h}] \leq_{\mathbf{m}_{1}} \mathbf{q}_{1}$ and $d \in \bar{d}_{0}\left[\mathbf{p}_{1}\left[\mathbf{h}^{\prime}\right].\right]$

Let $f^{\prime} \supseteq f_{\mathbf{h}}$ be as in $(*)_{1}(d)$ so in particular an isomorphism from $M_{1}$ onto $M_{2}$.

Now by clause (i) of Definition 4.16 there is $\mathbf{q}_{2} \in \mathscr{A}_{\mathbf{m}_{2}}$ such that $f^{\prime}$ maps $\mathbf{q}_{1}$ to $\mathbf{q}_{2}$.

The rest should be clear.]

$(*)_{8}$ if $\mathbf{h} \in \mathrm{AP}$ and $d \in N_{2} \underline{\text { then }}$ for some $\mathbf{h}^{\prime} \in \mathrm{AP}$ we have $\mathbf{h} \leq_{\mathrm{AP}} \mathbf{h}^{\prime}$ and $d \in \operatorname{Rang}\left(\bar{d}_{0}\left[\mathbf{p}_{1}\left(\mathbf{h}^{\prime}\right)\right]\right)$.

[Why? Like $(*)_{7}$.]

Together

$\odot$ there is a sequence $\left\langle\mathbf{h}_{i}: i<\lambda\right\rangle$ such that (for $\ell=1,2$ )

(a) it is $\leq_{\mathrm{AP}}$-increasing 
$(b)_{\ell}$ if $a \in M_{\ell}$ then $a \in B_{\ell}\left[\mathbf{h}_{i}\right]$ for some $i$

$(c)_{\ell}$ if $d \in N_{\ell}$ then $d \in \operatorname{Rang}\left(\bar{d}_{0}\left[\mathbf{p}_{\ell}\left(\mathbf{h}_{i}\right)\right]\right)$ for some $i<\lambda$.

From this sequence we can "read" an isomorphism as required, say $g\left(a_{1}\right)=a_{2}$ iff for some $i$ and $\varepsilon<\ell g\left(\bar{d}_{0}\left[\mathbf{p}_{1}\left[\mathbf{h}_{i}\right]\right)\right.$ we have $\left.a_{1}=\left(\bar{d}_{0}\left[\mathbf{p}_{1}\left[\mathbf{h}_{i}\right]\right]\right)_{\varepsilon}, d_{2}=\left(\bar{d}_{0}\left[\mathbf{p}_{2}\left[\mathbf{h}_{i}\right]\right]\right)_{\varepsilon} \cdot\right] \quad \square .20$ Another form, not assuming Hypothesis 4.12 is

Conclusion 4.21. Assume $(\lambda, \lambda,<\lambda)$ is T-good, e.g. $\lambda>|T|$ is a measurable cardinality and $\lambda=\lambda^{<\lambda}$. Then for some club $\mathbf{F}$ we have:

(A) (a) $\mathbf{F}$ is as in Sh:88r, 3.3], i.e.

( $\alpha) \mathbf{F}$ is a function with domain $\left\{\bar{M}: \bar{M}\right.$ has the form $\left\langle M_{i}: i \leq \beta\right\rangle$, $a \prec$-increasing continuous sequence such that $M_{i}$ is models of $T$ of cardinality $\lambda$ with universe an ordinal $\in\left[\lambda, \lambda^{+}\right)$and if $i$ is non-limit then $M_{i}$ is saturated $\}$,

( $\beta) \mathbf{F}(\bar{M})$ is such that $\bar{M}^{\wedge}\langle\mathbf{F}(\bar{M})\rangle \in \operatorname{Dom}(\mathbf{F})$

(B) $\bar{M}=\left\langle M_{\alpha}: \alpha<\lambda^{+}\right\rangle$obeys $\mathbf{F}$ which means that $\lambda^{+}=\sup \left\{\alpha: \mathbf{F}\left(\bar{M} \uparrow(\alpha+1) \prec M_{\alpha+1}\right\} \underline{\text { then }}\right.$

for some club $E$ of $\lambda^{+}$we have:

(a) $\operatorname{cf}(\alpha)=\lambda \Rightarrow M_{\alpha}$ is saturated,

(b) if $M_{\alpha_{\ell}}$ is saturated, $\operatorname{cf}\left(\delta_{\ell}\right)=\lambda$ and $\alpha_{\ell}<\delta_{\ell} \in E$ for $\ell=1,2$ then $\left(M_{\delta_{1}}, M_{\alpha_{1}}\right) \cong\left(M_{\delta_{2}}, M_{\beta_{2}}\right)$. 


\section{REFERENCES}

[CK73] Chen C. Chang and H. Jerome Keisler, Model Theory, Studies in Logic and the Foundation of Math., vol. 73, North-Holland Publishing Co., Amsterdam, 1973.

[Ehr57] Andrzej Ehrenfeucht, On theories categorical in power, Fundamenta Mathematicae 44 (1957), 241-248.

[ER69] Paul Erdös and Richard Rado, Intersection Theorems for Systems of Sets II, Journal of the London Mathematical Society 44 (1969), 467-479.

[Jec03] Thomas Jech, Set theory, Springer Monographs in Mathematics, Springer-Verlag, Berlin, 2003, The third millennium edition, revised and expanded.

[KM67] H. Jerome Keisler and Michael D. Morley, On the number of homogeneous models of a given power, Israel Journal of Mathematics 5 (1967), 73-78.

[KM78] Akihiro Kanamori and Menachem Magidor, The evolution of large cardinal axioms in set theory, Higher Set Theory, Lecture Notes in Mathematics, vol. 669, Springer - Verlag, 1978, pp. 99-275.

[Sh:c] Saharon Shelah, Classification theory and the number of nonisomorphic models, Studies in Logic and the Foundations of Mathematics, vol. 92, North-Holland Publishing Co., Amsterdam, xxxiv $+705 \mathrm{pp}, 1990$.

[Sh:3] _ Finite diagrams stable in power, Annals of Mathematical Logic 2 (1970), 69-118. [Sh:E53] _ Introduction and Annotated Contents, 0903.3428.

[Sh:72] _ Models with second-order properties. I. Boolean algebras with no definable automorphisms, Annals of Mathematical Logic 14 (1978), 57-72.

[Sh:88r] - Abstract elementary classes near $\aleph_{1}$, Chapter I. 0705.4137. 0705.4137.

[Sh:234] _ Classification over a predicate. II, Around classification theory of models, Lecture Notes in Mathematics, vol. 1182, Springer, Berlin, 1986, pp. 47-90.

[Sh:300] _ Universal classes, Classification theory (Chicago, IL, 1985), Lecture Notes in Mathematics, vol. 1292, Springer, Berlin, 1987, Proceedings of the USA-Israel Conference on Classification Theory, Chicago, December 1985; ed. Baldwin, J.T., pp. 264-418.

[Sh:300a] - Stability theory for a model, Chapter V (A), in series Studies in Logic, vol. 20, College Publications.

[Sh:715] _ Classification theory for elementary classes with the dependence property - a modest beginning, Scientiae Mathematicae Japonicae 59, No. 2; (special issue: e9, 503544) (2004), 265-316, math.LO/0009056.

[Sh:783] - Dependent first order theories, continued, Israel Journal of Mathematics 173 (2009), 1-60, math.LO/0406440.

[Sh:863] - Strongly dependent theories, Israel Journal of Mathematics accepted, math.LO/0504197.

[Sh:868] When first order T has limit models, Colloquium Mathematicum 126 (2012), 187-204, math.LO/0603651.

[Sh:877] _ Dependent $T$ and Existence of limit models, Tbilisi Mathematical Journal submitted, math.LO/0609636.

[Sh:886] _ Definable groups for dependent and 2-dependent theories, Journal of Symbolic Logic submitted, math.LO/0703045.

[Sh:906] , No limit model in inaccessibles, CRM Proceedings and Lecture Notes 53 (2011), 277-290, 0705.4131.

[KpSh:946] Itay Kaplan and Saharon Shelah, A counterexample to existence of indiscernibles and other examples, Journal of Symbolic Logic accepted.

[Sh:950] Saharon Shelah, Dependent dreams: recounting types, 1202.5795.

[Sh:F1127] — Ultrafilters on $\aleph_{1}$ generated by few conditions.

Einstein Institute of Mathematics, Edmond J. Safra Campus, Givat Ram, The HeBrew University of Jerusalem, Jerusalem, 91904, Israel, and, Department of Mathematics, Hill Center - Busch Campus, Rutgers, The State University of New Jersey, 110 Frelinghuysen Road, Piscataway, NJ 08854-8019 USA

E-mail address: shelah@math.huji.ac.il

URL: http://shelah.logic.at 